\title{
WestVirginiaUniversity
}

THE RESEARCH REPOSITORY @ WVU

Graduate Theses, Dissertations, and Problem Reports

2003

\section{Adding liveness detection to the hand geometry scanner}

Musat C. Crihalmeanu

West Virginia University

Follow this and additional works at: https://researchrepository.wvu.edu/etd

\section{Recommended Citation}

Crihalmeanu, Musat C., "Adding liveness detection to the hand geometry scanner" (2003). Graduate Theses, Dissertations, and Problem Reports. 1369.

https://researchrepository.wvu.edu/etd/1369

This Thesis is protected by copyright and/or related rights. It has been brought to you by the The Research Repository @ WVU with permission from the rights-holder(s). You are free to use this Thesis in any way that is permitted by the copyright and related rights legislation that applies to your use. For other uses you must obtain permission from the rights-holder(s) directly, unless additional rights are indicated by a Creative Commons license in the record and/ or on the work itself. This Thesis has been accepted for inclusion in WVU Graduate Theses, Dissertations, and Problem Reports collection by an authorized administrator of The Research Repository @ WVU. For more information, please contact researchrepository@mail.wvu.edu. 


\section{Adding liveness detection to the Hand Geometry Scanner}

Musat C. Crihalmeanu

Thesis submitted to the College of Engineering and

Mineral Resources at West Virginia University

In partial fulfillment of the requirements for the degree of:

Master of Science in Electrical Engineering

Committee Members:

Mark A. Jerabek, Ph.D., Chair

Wils L. Cooley, Ph.D.

Lawrence A. Hornak, Ph.D.

Dimitris Korakakis, Ph.D.

West Virginia University

Lane Dept. of Computer Science and Electrical Engineering

Morgantown, West Virginia

2003

Keywords: Biometrics, Plethysmography, Pulse Oximetry, Optical Sensors, Micro-controller 


\section{ABSTRACT}

\section{Adding Liveness Detection to the Hand Geometry Scanner}

\section{Musat C. Crihalmeanu}

In today's dynamic society, the efficiency of the Biometric Systems has an increasing tendency to replace the classic but obsolete keys and passwords. Hand Geometry Readers are popular biometrics used for Access and Control applications. One of their weaknesses is vulnerability to spoofing using fake hands (latex, play-doh or dead-hands).

The objective of this Thesis is to design a feature to be added to the Hand Geometry Scanner in order to detect vitality in the hand, reducing spoofing possibilities.

This thesis demonstrates how the Hand Reader was successfully spoofed and shows the implementation of the live detection feature through an inexpensive but efficient electronic design.

The method used for detection is Photo-Plethysmography. The Reflectance Sensor built is of original conception. After amplifying, filtering and processing the sensor's signal, a message is displayed onto an LCD, concerning the liveness of the hand and the pulse rate. 


\section{ACKNOWLEDGEMENTS}

1) I want to address special thanks to Simona, my wife, for her sacrifice and loving support and I dedicate this work to her.

2) Thank you to LDCSEE Department for the excellent conditions provided during my Research.

3) Thank you to Dr. Mark .A. Jerabek, my advisor, for his guidance, patience and encouragements during the course of the research.

4) Thank you to Dr. Kathleen. Meehan, my first advisor, who came up with the generous idea of this project.

5) Thank you to Dr. Lawrence Hornak and Dr. Bojan Cukic for the sponsorship of the last semester of Research. Without it, this work wouldn't be possible.

6) Thank you to Dr. Wils L. Cooley, Dr. Lawrence A. Hornak and Dr. Dimitris Korakakis, for serving in my comitee.

7) Thank you to Mr. Vikram Bose-Mullick, graduate student, who encouraged me to dare to use the PIC MCUs everywhere, and supported me with hints and enthusiasm.

8) Thank you to Mr. Pisut Raphisak, Ph.D student from BIOSAL Lab., for his hints in ECG signal processing and adaptive triggering. 


\section{TABLE OF CONTENTS}

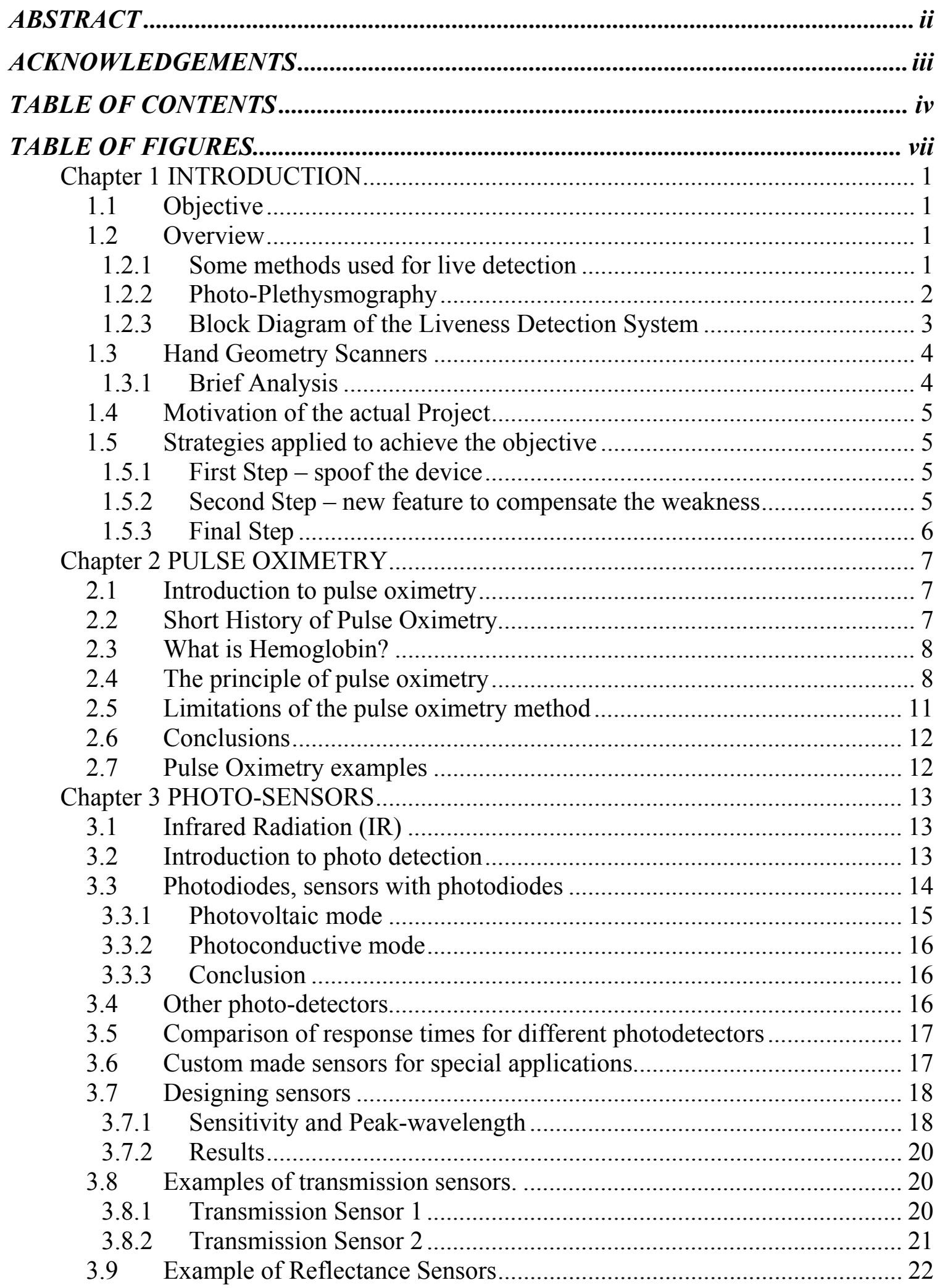


3.9.1 Single Pair Reflectance Sensor ......................................................... 22

3.9.2 Special Multi-emitter Sensor …………………................................. 23

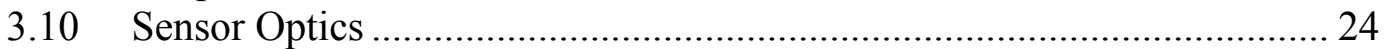

Chapter 4 PREAMPLIFIERS FOR OPTICAL SENSORS ..................................... 26

$4.1 \quad$ Introduction on preamplifiers. ........................................................... 26

4.2 Circuits used as Amplifiers for sensors .................................................. 27

4.2.1 For the normal photodiode and PIN photodiodes …………………..... 27

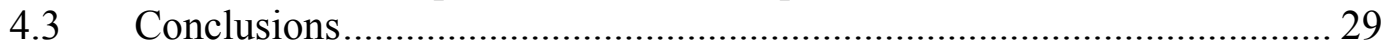

Chapter 5 FILTERING UNWANTED FREQUENCIES ....................................... 30

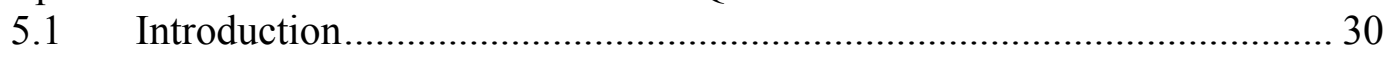

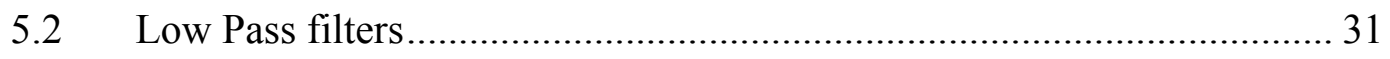

5.3 The Main Characteristics of a Second Order Filter ................................... 31

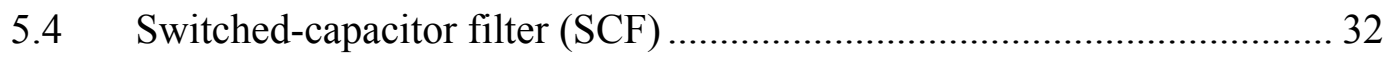

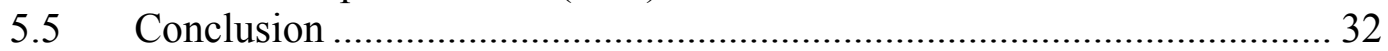

5.6 Choosing the filter for the actual application.......................................... 32

Chapter 6 SIGNAL PROCESSING ..................................................................... 34

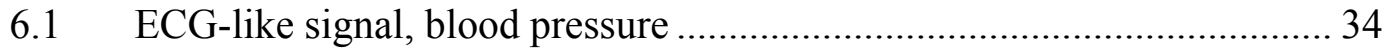

6.1.1 Heart Mechanism................................................................................. 34

6.1.2 ECG propagation along the whole body …………………………..... 35

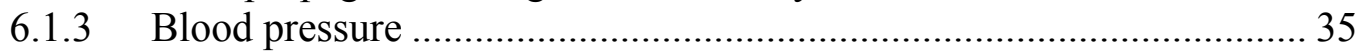

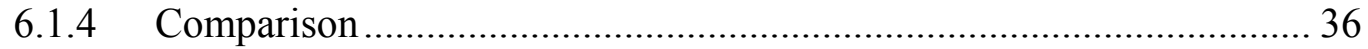

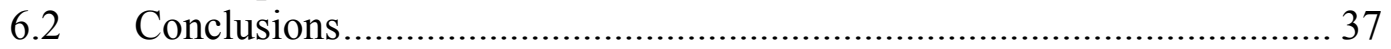

6.3 Processing the ECG signal to decide upon liveness …………………....... 37

6.3.1 QRS complex recognition................................................................... 37

6.3.2 Brief example of a typical method of parameters determination........... 37

6.4 Important ideas for our project (R-peak detection)................................... 39

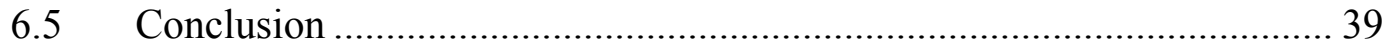

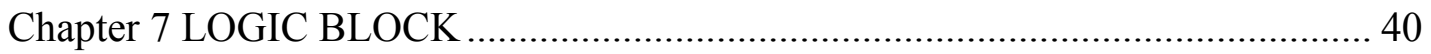

$7.1 \quad$ Block Diagram .................................................................................. 40

7.2 The presentation of the details of the schematic of the logic block ........... 41

7.2.1 LM 311 brief presentation .............................................................. 42

7.2.2 SN74LS14 (Hex Inverter, Schmidt Trigger) ……………………….... 43

7.2.3 LM 555 (Monostable configuration) ……………………………........ 44

7.3 Analog and Logic, Schematic and PCB (practical considerations) .......... 45

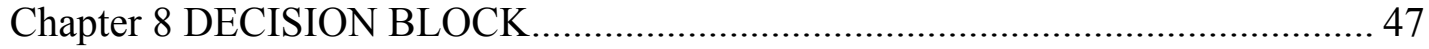

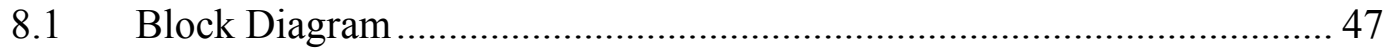

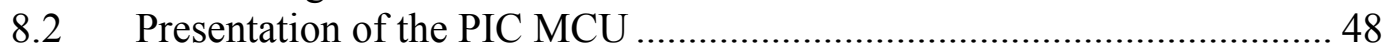

8.2.1 Microcomputer and Micro-controller ................................................... 48

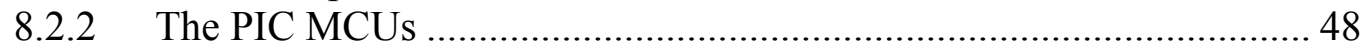

8.2.3 PIC's basic arhitecture and other features ........................................... 49

8.2.4 Some Disadvantages of PICs …………………….............................. 49

8.2.5 Brief overview of PIC 18F252 …………………......................... 50

8.3 LCD (Liquid Crystals Display) - Optrex DMC20434 ………………....... 50

8.3.1 General characteristics .................................................................... 50

8.3.2 Operation modes ........................................................................... 51

8.3.3 Initialization of the LCD. Building an LCD driver............................... 52 
8.3.4 Connecting the Optrex DMC 20434 LCD with the PIC..................... 52

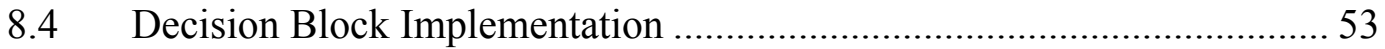

8.4.1 Version 1 (Roll over measurement) .................................................... 53

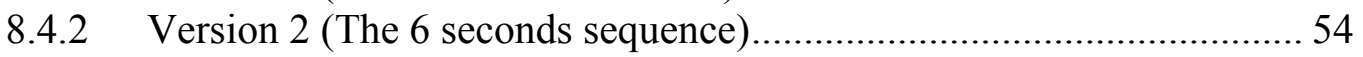

8.4.3 PSpice simulation for the Monostables 1 and 2 ............................... 56

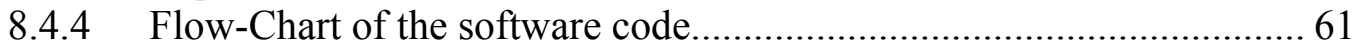

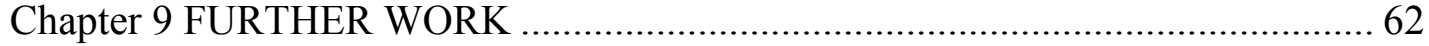

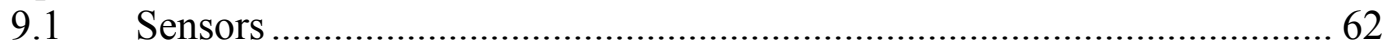

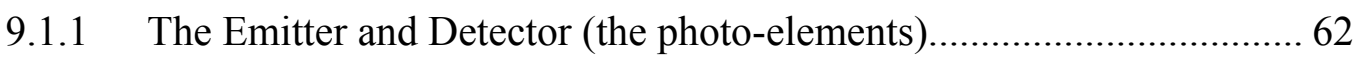

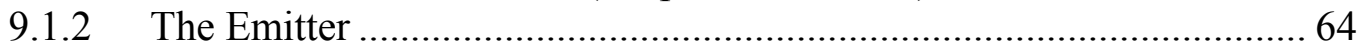

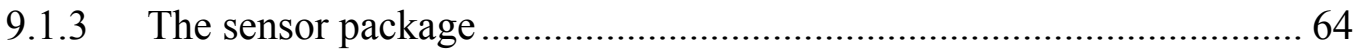

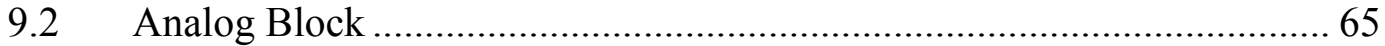

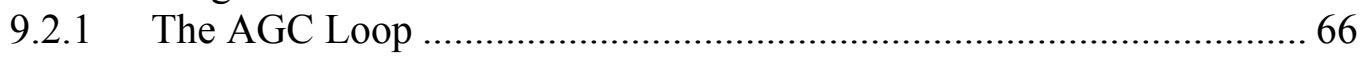

9.2.2 Decisional block improvements .............................................. 70

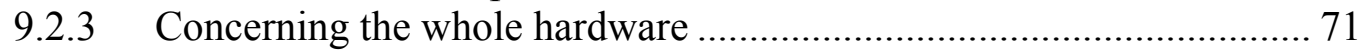

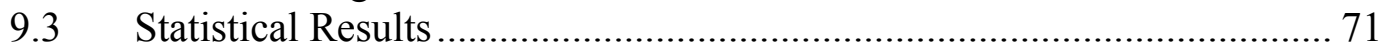

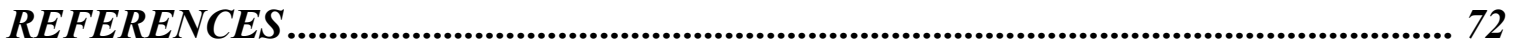

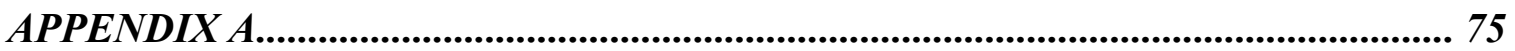

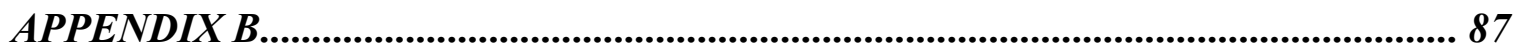

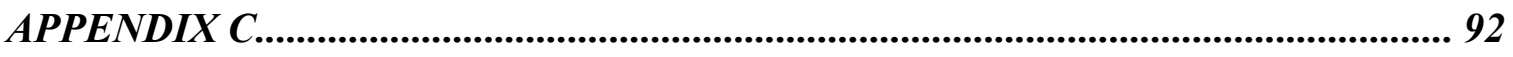




\section{TABLE OF FIGURES}

Fig.1.1 Detections through photo-plethysmography in a fingertip ................................ 2

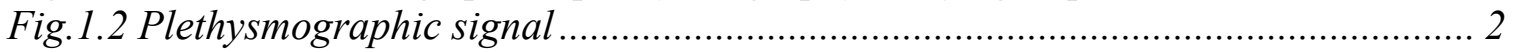

Fig.1.3 Block Diagram for the Liveness Detection System ......................................... 3

Fig.1.4 Flow Chart showing how the new feature (Live Detection) can interact and work

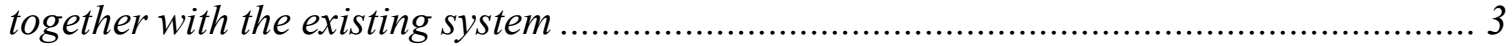

Fig.1.5 HK2-hand geometry scanner (photos from: www.handreader.com) ................... 4

Fig.2.1 Light absorbance at two wavelengths: 660nm and 910nm.(from [12])................ 8

Fig.2.2 Variable light absorption due to pulsatile volume of arterial blood.(from [12]) ... 9

Fig.2.3 Transmission pulse oximetry (from [12]).................................................... 9

Fig.2.4 Relationship between Arterial Pressure of Oxygen and Arterial Oxygen

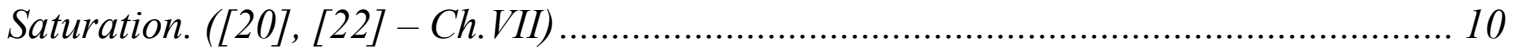

Fig.3.1 The electromagnetic spectrum - main regions (source [31]) ............................. 13

Fig.3.2 Photodiode equivalent circuit ................................................................. 14

Fig.3.3 Connection of a photodiode in a photovoltaic mode ......................................... 15

Fig.3.4 Photoconductive operating mode ............................................................... 16

Fig.3.5 Relative spectral sensitivity of PNZ334 silicon photodiode and the peak

wavelength emissions of several types of LEDs....................................................... 18

Fig.3.6 Opto-Interrupter Switch from Fairchild Semiconductor, H22B1 ..................... 19

Fig.3.7 Schematic for a Trans-impedance Amplifier with photo-Darlington and its output

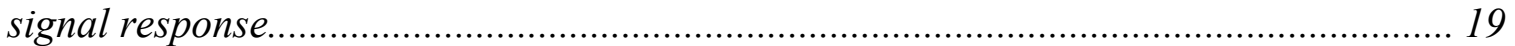

Fig.3.8 Technical drawing with the Transmission Sensor 1....................................... 20

Fig.3.9 Photograph with the ABS plastic, Transmission Sensor 1, in center.................. 21

Fig.3.10 A Technical drawing for Transmission Sensor 2 ........................................ 21

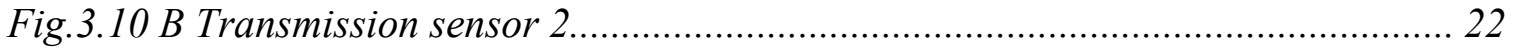

Fig.3.11 Single Pair Reflectance Sensor .......................................................................... 22

Fig.3.12 Single pair reflectance sensor embedded in a wooden platen .......................... 23

Fig.3.13 Multi-emitter Sensor (Reflectance Sensor 2).................................................. 23

Fig.3.14 Picture with Reflectance Sensor 2 (multiple emitters) .................................... 24

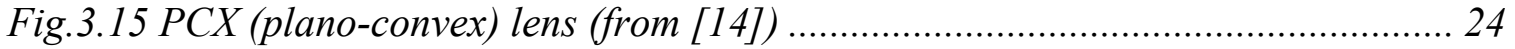

Fig.3.16 The figure explains how the coating may be chosen, and what is the expected range of wavelengths transmitted and stopped.(from [14]) ........................................ 25

Fig.4.1 Trans-impedance Amplifier (Current Controlled Voltage Source)...................... 26

Fig.4.2 Op Amp circuit used for PIN photo diodes ....................................................... 27

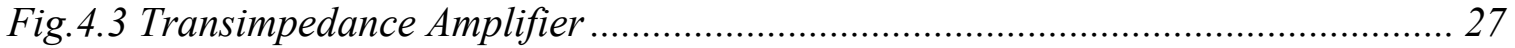

Fig.4.4 Second stage (Voltage Amplifier) with INA 126 ............................................ 28

Fig.4.5 Trans-impedance Amplifier for phototransistors ........................................... 29

Fig.5.1 Low-Pass Filter Responses, according with the approximation used: a) Ideal, b)

Butterworth, c) Bessel, e) Chebysev, f) Elliptic; (from [24]).......................................... 31

Fig.5.2 Low Pass $8^{\text {th }}$ order SCF, dual power supply filter.......................................... 32

Fig.5.3 The $1 \mathrm{KHz}$ clock generator for the SCF build with Max 291 ............................. 33

Fig.5.4 The Low Pass Filter together with its external components ............................. 33

Fig.6.1 Standard ECG ........................................................................................ 34

Fig.6.2 Blood Pressure Wave-shape (from [32], page5)........................................... 35 
Fig.6.3 Standard ECG Signal, main features used in physiology (from [32],

http://www.ee.tut.fi/rgi/kurssit/71221/ekg_analyysi/ECGANALY.html )....................... 36

Fig.6.4 Photo-Plethysmographic wave-shape, obtained through photo-sensors............. 36

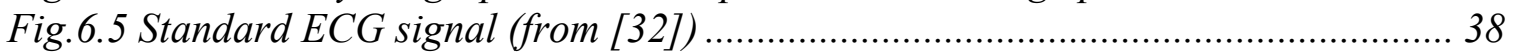

Fig. 7.1 Block Diagram of the whole Project .............................................................. 40

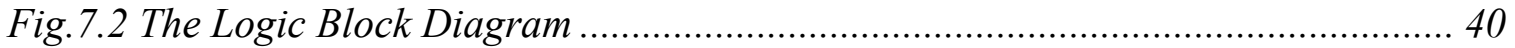

Fig.7.3 The input \& output signals of the comparator LM 311, for a threshold $=1 \mathrm{~V} \ldots . .40$

Fig.7.4 Detailed electronic schematic of the Logic Block and the last stages of the Analog Block .............................................................................................................. 41

Fig.7.5 LM 311, detail with the Open Collector output.............................................. 42

Fig.7.6 Internal organization of the Hex inverter SN 74LS14................................... 43

Fig. 7.7 Left: LM555 - Monostable Standard configuration Right: Input (2), Output (3) and Discharge (7) Capacitor Waveforms (from data sheet) ...................................... 44

Fig. 7.8 Detailed Schematic, Analog plus Logic Blocks............................................. 45

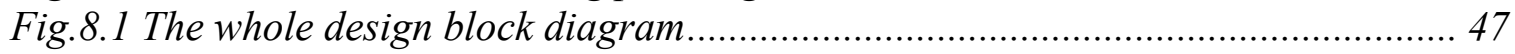

Fig.8.2 Flow Chart for Decision Block Software ...................................................... 53

Fig.8.3 Detailed Electronic Schematic for the Decision Block ..................................... 54

Fig.8.4 Flow Chart with the explanation of Decision Block Schematic .......................... 54

Fig.8.5 PSpice schematic of Monostable 1 from the Decision block............................. 56

Fig.8.6 Represents: Vout (cyan, pin 3 LM555) vs Vin (magenta, pin 2 LM555)............. 57

Fig.8.7 The output of the Monostable 1 (6 seconds, after the transition High to Low of the

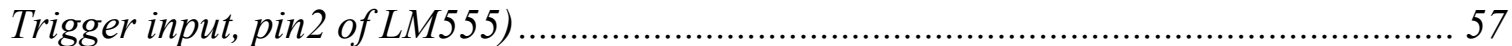

Fig.8.9 Represents Vouptut (cyan, pin3 LM555, To $=25 \mathrm{~ms}$ ) vs Vinput (magenta, pin2

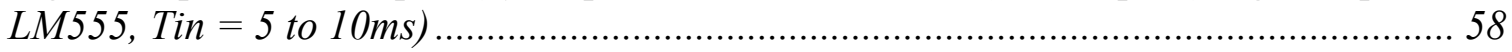

Fig.8.10 Logic and part of Decision Block interconnections (without PIC and LCD) ... 59

Fig.8.11 Above- the outputs from the binary Counter ( $Q A, Q B, Q C, Q D)$ Center - Red: output of M1 (6sec.); Green: output of M2 (10ms)...................................................... 59

Fig.8.12 The signal output from the monostables, acquired from the Oscilloscope ....... 60

Fig.8.13 Flow-Chart for the software version 2, Decisional Block............................... 61

Fig.9.1 Oxyhemoglobin (red) and Deoxyhemoglobin (violet) absorbance versus

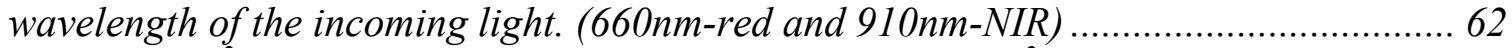

Fig.9.2 $3 \mathrm{~mm}^{2}$ photodiode. Stock $\mathrm{nr}$. NT 55-755; Area $=3 \mathrm{~mm}^{2} ;$ Price $=\$ 290.00$ [14] .. 63

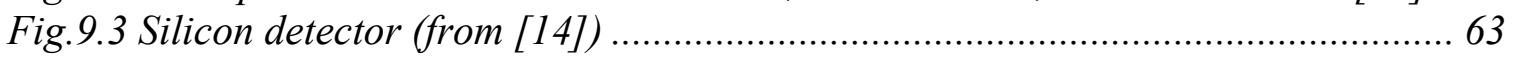

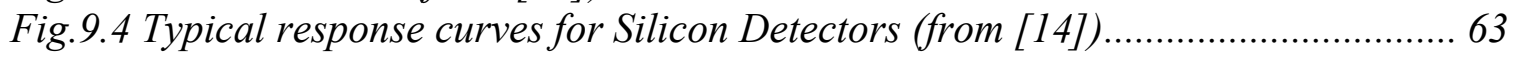

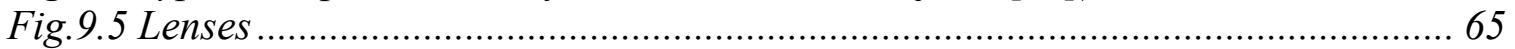

Fig.9.6 Replacing R8 (gain of IC2A-OPA2134) with a PGA (programmable gain

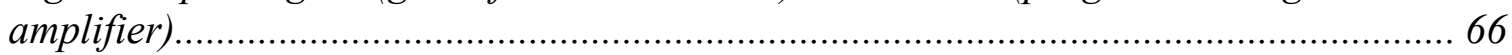

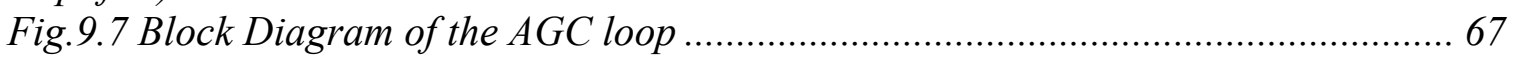

Fig.9.8 More detailed Schematic of the AGC block .................................................... 67

Fig.9.9.A Peak Detector with diode and capacitor, and B Peak Detector using an Op

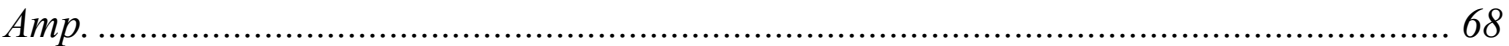

Fig.9.9.C Classic peak detector with op amps and diode (rectifier and follower).......... 68

Fig.9.10 Peak Detector with LM311, typical application from National Semicond ........ 69 


\section{Chapter 1 INTRODUCTION}

\subsection{Objective}

The Hand Geometry Scanner "Hand-Key II" (Recognition Systems) is an accurate device used for identification purposes; one of many Access and Control or Time and Attendance devices. One of the issues facing hand geometry scanning biometric systems is that they can be fooled by spoof devices (false hands - latex, playdo, etc.) or dead hands.

The objective of this project is to design a feature to be added to the Hand Geometry Scanner, in order to determine the vitality of the hand, reducing the possibility of spoofing.

\subsection{Overview}

This project was developed during a research done under the guidance of Dr. Mark Jerabek (LDCS\&EE - West Virginia University) and was sponsored in part by DoD, the Department of Defense. The goal was to build an anti spoofing system, as inexpensive as possible (small power consumption, small volume and cheap components) to be added to the existing biometric device HK2, made by Recognition Systems, in order to increase the security of the system.

Key Words used in this thesis and their explanation: Biometrics, Spoofing devices, Plethysmography, Photo-plethysmography, Pulse Oximetry.

Biometrics: (eng. approach) Reduce the problem of confirming a person's identity to the problem of authentication of a concrete entity related to a person. (Dr. L. A. Hornak "Biometric Systems, 5 Day Course")

Spoofing Devices: Devices build with the intention to fool a biometric system

Plethysmograph: An instrument that measures variations in the size of an organ or body part, due to respiration or blood circulation.

Photo plethysmograph: A Plethysmograph using photo transducers.

Pulse Oximetry: A measure of oxygen saturation in arterial blood.

\subsubsection{Some methods used for live detection}

To the best of our knowledge these are some liveness detection methods applicable to the human hand:

1. Plethysmography - through: optical sensors, piezo-electrical sensors (or other pressure sensors), microwave Doppler radar (for circulatory and respiratory movements).

2. Spectrometry - measuring the absorbance of specific wavelengths of light (used in Pulse Oximetry).

3. Temperature - measuring the heat emitted by a specific part of the body. The efficiency of this method is reduced because a fake hand can be reheated to the level of human body temperature very easily. 
4. Skin Resistivity - maps the resistivity of a number of points from the palm. One of the drawbacks of using this method is that the skin surface resistivity of the hand is constantly changing due to the direct physical contact with human activities.

5. RF (radio-frequency) bio-effects - emitting and receiving RF signals to and from an object or body, offers information about liveness in that object or body. Adjusting the exposure level at non-harmful values can create difficulties.

6. Perspiration - using the characteristic of the living skin to have more or less moisture on it (used for finger vitality for fingerprint scanner anti-spoof).

\subsubsection{Photo-Plethysmography}

The method chosen for life detection in this thesis is Photo-plethysmography, getting a pulse measurement using Optical sensors (Photo Emitters and Photo Detectors). The amount of light reflected from the finger bone and received by the detector depends on the limb volume, wavelength of the light beam and scattering properties of the tissue in the light path (fig.1.1):

\section{Reflectance photo-plethysmography}

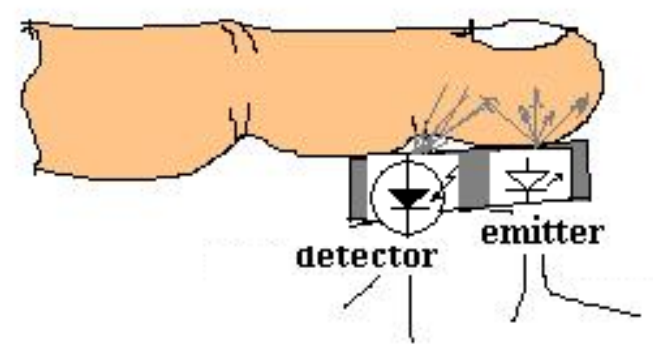

Fig.1.1 Detections through photo-plethysmography in a fingertip

The signal shape obtained from photo-plethysmography looks like in fig.1.2:

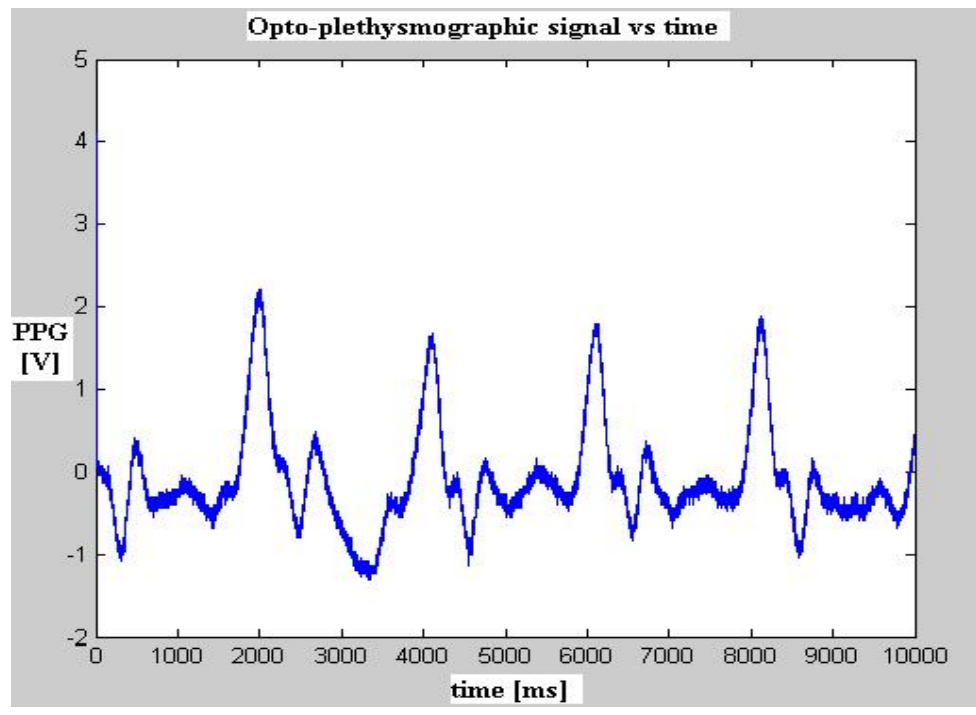

Fig.1.2 Plethysmographic signal 


\subsubsection{Block Diagram of the Liveness Detection System}

The design consists mainly of four blocks:

1. The Sensor Block will be mounted into the platen of the HK2.

2. The Analog Block contains amplifiers and filters.

3. A Logic Block which plays the role of interfacing with the Decision Block.

4. The Decision Block, consisting of a very simple embedded system designed to count, process pulses obtained from the hand and display a decision using an LCD: Live detected, the subject is alive or Try Again, the subject is suspect and access should be denied for that try.(see Fig. 1.3)

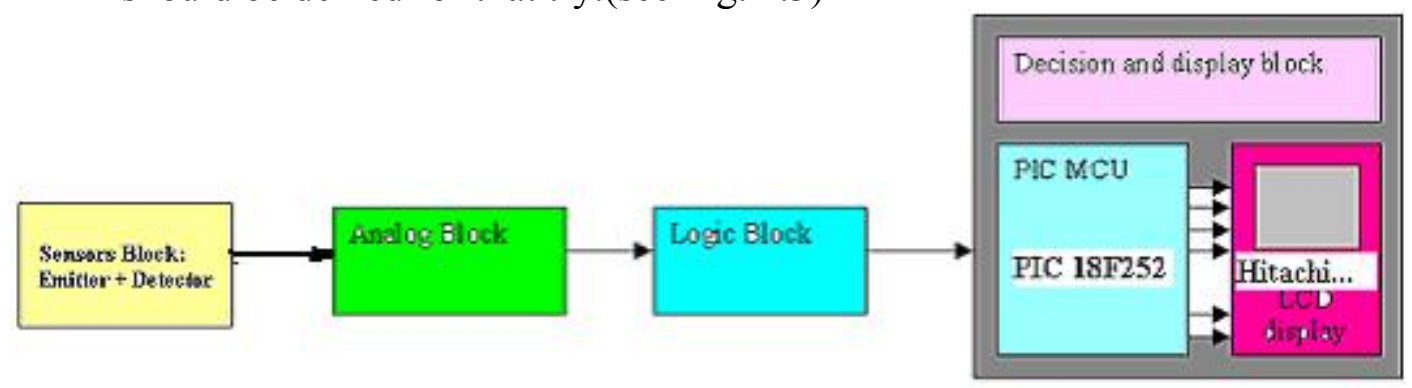

Fig.1.3 Block Diagram for the Liveness Detection System

For a better understanding of the new feature (Live detection) consider the following flow chart:

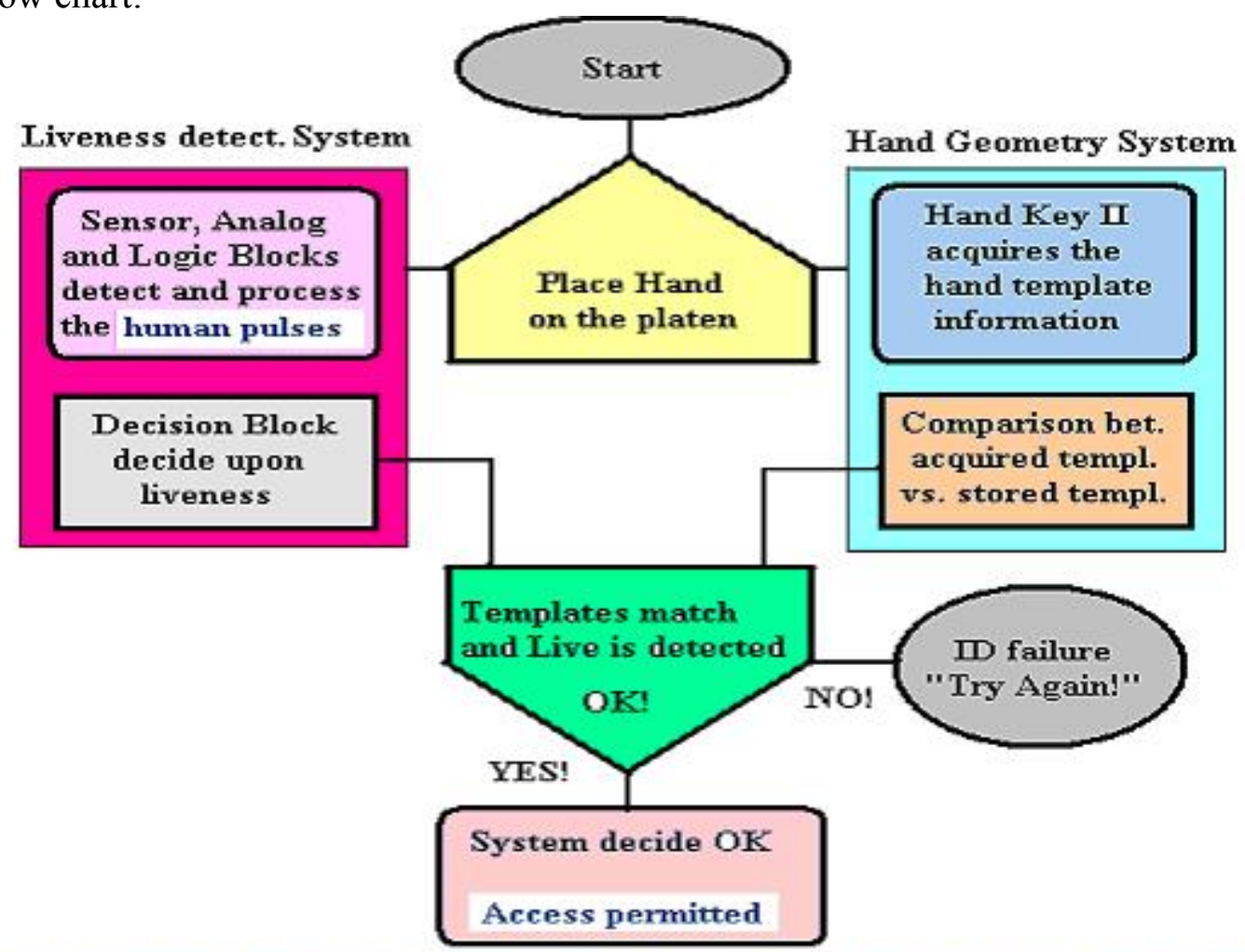

Block Diagram Showing the interaction of HandKey 1 with the Live detect System Fig.1.4 Flow Chart showing how the new feature (Live Detection) can interact and work together with the existing system 


\subsection{Hand Geometry Scanners}

\subsubsection{Brief Analysis}

Hand Geometry Reader authentication provides a reliable, fast and user-friendly alternative to password inefficiency, which requires the user to remember and enter cumbersome and often numerous code combinations.

35 years ago, Shearson Hamil Investment Bank on Wall Street used the first Hand Geometry Reader. In recent years it has become a very popular access control biometric. It is a "friendly" biometric, non-intrusive, with a high collectability, medium universality, uniqueness and permanence, and small access time. According to "Biometric Technology Today" (www.sib.co.uk): "The Hand Geometry Reader has captured almost half of the physical access control market" (!).

The hand scanner is based on the idea that some features related to the human hand (length, width, thickness, height or straightness of the fingers and palm) are relatively invariant and peculiar to each individual.

The "Hand key2"(HK2) is Recognition Systems' fourth generation biometric, Access and Control hand reader. The hand reader records the 3D shape of the hand using infrared light imaging technique, builds and stores a 9 byte template from the main features of the hand, finally being able to unlock a door or communicate with a host computer (for details see [2]).
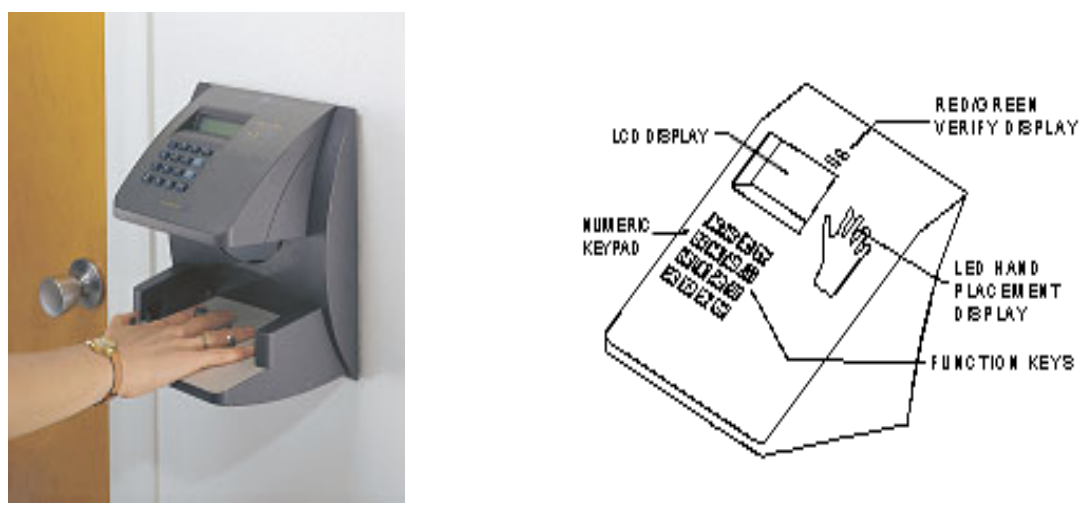

Fig.1.5 HK2-hand geometry scanner (photos from: www.handreader.com)

Recognition Systems Inc. (www.recogsys.com ), a division of Ingersoll-Rand (ranked 205 in Fortune 500), is the world wide leader in Biometric Access Control, Time and Attendance and Personal Authentication Products. The company was founded in 1986 and has pioneered the commercialization of biometrics using its patented hand geometry technology, that identifies people by the size and shape of their hand.

From the point of view of Acceptability, Dick Zunkel (Recogn. Systems) says: "Among biometric systems, Sandia Laboratories reported that Hand Geometry Scanners have the highest user acceptance of all devices tested. ..." 


\subsection{Motivation of the actual Project}

There is no perfect biometric system. Each system has its advantages and disadvantages. The user of a biometric system can use various criteria of selection, such as performance, acceptability, resistance to circumvention, FAR (false acceptance ratio) / FRR (false rejection ratio), etc., trying to match it as closely as possible to meet the needs of his specific application. Usually, for medium secure applications, medium criteria will satisfy; but when security becomes an issue (banks, power plants, military facilities and others) only a high level of those criteria will be satisfactory

The weak point of the hand reader is the Resistance to Circumvention that is considered only medium. The hand readers don't sense the life of the hand, they build a mathematical template just using the geometrical features of the palm and fingers. A plastic (latex) hand, accurately cast can easily spoof these devices. Also a dead hand can spoof it as well.

This is a problem when considering access to high security areas.

Adding liveness to the hand geometry technology, will ensure a higher Resistance to Circumvention feature, thus increasing the performance of the hand geometry scanners. Doing this without compromising the desirable features of the hand scanner is the goal of the work of this Thesis.

\subsection{Strategies applied to achieve the objective}

\subsubsection{First Step - spoof the device}

As mentioned before, no biometric is perfect, but finding exactly where are its weak points or features and trying to eliminate those weaknesses by adding new features, is not easy to achieve. In the case of the Hand Reader, research has been done to find the appropriate material and technology to be used for the cast of the hand and for the mold of the hand. Finally the most inexpensive combination (cast and mold) will be picked for demonstrating the weakness (gaining false access, e.g.).

\subsubsection{Second Step - new feature to compensate the weakness}

a) A sensor to detect life in the hand has to be designed. Research has to reveal the most modern methods in use for life detection. New ideas have to be taken in account for the success of this project.

b) An analog block will amplify the signal obtained at the output of the sensor, in order to bring its magnitude to a level suitable for signal processing. The gain of the amplifiers will be carefully calculated. Filters have to be designed to purify the useful signal from the inherent noise.

c) An algorithm to process the analog signal then has to be designed.

d) A Decision Block will further analyze the results of processing, and the final decision upon liveness will be displayed: "Live Detected!" or "Try Again!" accordingly. 


\subsubsection{Final Step}

The objective of this work is to add liveness detection without compromising the performances of the HK2 hand geometry scanner. No infrared interference has to be made from above or from the side of the hand placed on the platen. This would interfere with the infrared imaging optics of the scanner, drastically affecting the accuracy of the geometrical template built by the system. Since the reflectance sensor built to detect liveness lies under the palm, completely shadowed by the hand, and the emitting power of the emitter is just $10 \mathrm{~mW}$, linearly decreasing with the distance (the CCD (charge coupled device) camera is $>120 \mathrm{~mm}$ from the platen), the interference is considered negligible.

Another feature that has to be controlled in order not to affect the acceptability criteria of a biometric is the time that the hand is required to be kept on the platen. This time interval has to be the same or less then the HK2 time interval needed for template generation and comparison, that is, approximately 3 seconds (on average).

Movement artifacts of the palm or fingers can deteriorate, both, the template generation and liveness detection feature. The platen contains five pins to be squeezed during template generation. If the user moves the palm or the fingers during the template generation, this user will be rejected. Based on this, less care should be taken regarding the movement artifacts that might compromise the live detection feature.

The final step is implementing the new feature to work together with the existing device and give statistical proof of achieving the goal initially stated: to compensate for the weak resistance to circumvention. 


\section{Chapter 2 PULSE OXIMETRY}

The goal of this chapter is to show in detail the relationship between pulse oximetry and plethysmography, the advantages and drawbacks of the pulse oximetry liveness detection method. This chapter purpose is to reduce the confusion around pulse oximetry and plethysmography related terms.

\subsection{Introduction to pulse oximetry}

Pulse oximetry is a simple, non-invasive method of monitoring the oxygen saturation of arterial capillary blood. The sensing method applied by Pulse Oximetry is Spectrometry, theoretically based on the Lambert-Beer Law and on the fact that Oxihemoglobin and Dioxihemoglobin absorb differently at specific wavelengths of light.

Pulse Oximetry is now widely used in a number of health care settings and is often referred to as the "fifth vital sign".

The pulse oximeter is a convenient, cost-effective way to monitor the patient oxygenation status and determine changes before they are clinically apparent.

\subsection{Short History of Pulse Oximetry}

(adapted from: [11] and [22], Chapter III.)

In 1875, a German physiologist name Karl von Vierofdt demonstrated that the oxygen in his hand was consumed when a tourniquet was applied. ("A Tourniquet is a device (as a bandage twisted tight with a stick) to check bleeding or blood flow "-[21]). This was done utilizing transmitted light waves, but the development of the pulse oximeter was still long way off. In 1936 Karl Matthes developed the first ear saturation meter that used two wavelengths of light. This compensated for the variations in tissue absorbtion. This idea was improved in 1940, when Glen Millikin developed a lightweight oximeter to help the military solve their aviation hypoxia problem.

"The modern pulse oximetry was developed by Takuo Aoyagi, (Nihon Koden) in Tokyo, while developing a non-invasive cardiac output measurement, using dye dilution and an ear densitometer. He noticed a correlation in the difference between the unabsorbed infrared and red light, and the oxygen saturation. This led to the clinical application of the pulse oximeter..." ([22], Ch.III.)

In the late 70s Hewlett Packard engineers designed some pulse oximeters, but it was not until the1980's (discussions) that Nellcor and Biox produced the first commercially available pulse oximeters, that were reliable, robust and affordable.

"In 1988 the use of the pulse oximeter during anesthesia and recovery became mandatory in Australia. Since then, its use has become mandated in many areas, from pre-hospital treatment to intensive care units..." ([22], Ch.III). 


\subsection{What is Hemoglobin?}

The hemoglobin molecule consists of 10,000 atoms, four of which are iron atoms that attract and hold oxygen. Each red blood cell contains about 250 million hemoglobin molecules. There are approximately 5,000 cc's of blood in the average individual and each cc contains five billion red blood cells. When oxygen is bound to the hemoglobin, it is called "oxyhemoglobin". ([22],Ch.VI)

Oxygen is a clear odorless gas that accounts for $21 \%$ of the gases around us. It is essential for the process our body uses to produce energy needed for metabolism. Too much (hyperoxia) or insufficient oxygen (hypoxia) can cause illness or even death. Therefore it is necessary to be able to measure the amount of oxygen in the blood. In the past, the only method of measuring oxygen saturation was via an ABG (arterial blood gas). This method was hard to apply, first because it was done through an invasive process.

\subsection{The principle of pulse oximetry}

Pulse Oximetry is a standard of care for monitoring arterial oxygen saturation during sedation. Maintaining an arterial oxygen saturation above $90 \%$ assures a $\mathrm{PO}_{2}$ of 60 $\mathrm{mmHg}$ or greater, will prevent hypoxemia (the lack of arterial oxygen saturation), enabling an early detection of life-threatening events. An oxygen saturation under $85 \%$ $(55 \mathrm{mmHg})$ can be the signal of a light Cyanosis. At $75 \%$ to $80 \%$ - moderate Cyanosis and at $70 \%$-definte Cyanosis (at $40 \mathrm{mmHg}$ ). Cyanosis is a very late sign, meaning severe hypoxemia.[11],[12],[22]. The pulse oximeter utilizes 2 LEDs that emit light: one with a wavelength in the red band $(660 \mathrm{~nm})$ and another in the infrared band $(910 \mathrm{~nm})$.

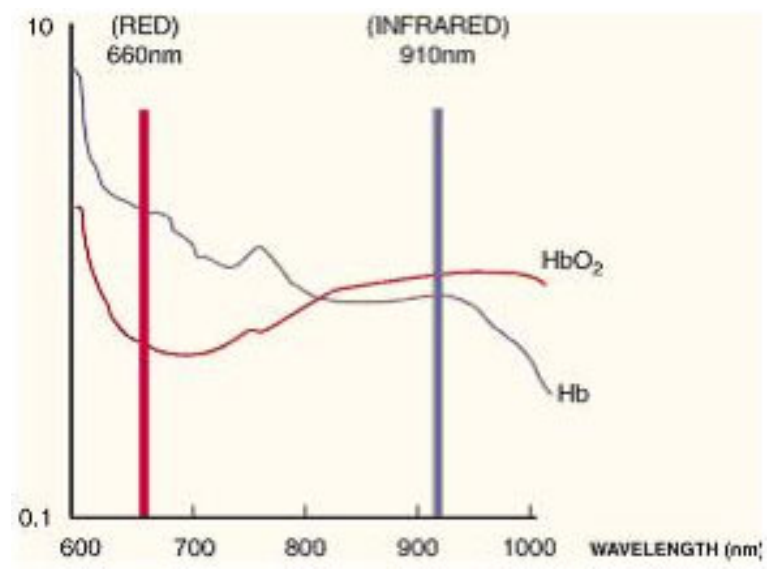

Fig.2.1 Light absorbance at two wavelengths: 660nm and 910nm.(from[12])

The photodetector, which should be positioned precisely opposite the emitter diodes (tramsnission method), measures the differences in light absorbance at the 2 wavelengths for oxyhemoglobin and deoxyhemoglobin. (See Fig.2.1) Using spectrophotometry, this ratio is measured and then a computer calculates the functional saturation of hemoglobin: 
Fuctional sat. of hemoglobin $=[$ oxyhemogl. $/($ oxyhemogl. + reduced $\mathrm{Hb})] \times 100$

Pulse oximetry based monitors utilize a plethysmographic waveform to differentiate pulsatile arterial hemoglobin saturation from venous blood. (See Fig.2.2)

The absence of a pulsatile waveform (as in hypothermia or hypoperfusion) interferes with the oxymeter's function. If plethysmography is not able to track the arterial pulse, the oxymeter does not correlate with the HR (Heart Rate) on the ECG, so the accuracy of oxygen saturation may be low.

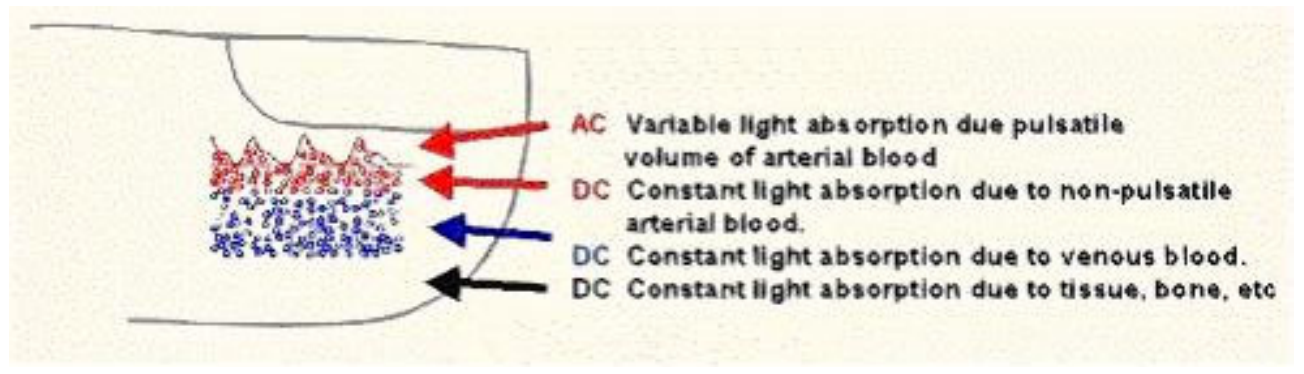

Fig.2.2 Variable light absorption due to pulsatile volume of arterial blood.(from[12])

The red and IR LEDs shine their light through a reasonably translucent site with a good blood flow. Typical sites: finger, toe, and lobe of the ear. The photo-detector stays at the opposite side, receiving the amount of radiation that passes through. Basically one can use two methods for sensing pulse oximetry: Transmission and Reflectance.

Transmission method applies when the emitter and detector are on opposite sides of the measuring site. (See Fig.2.3)

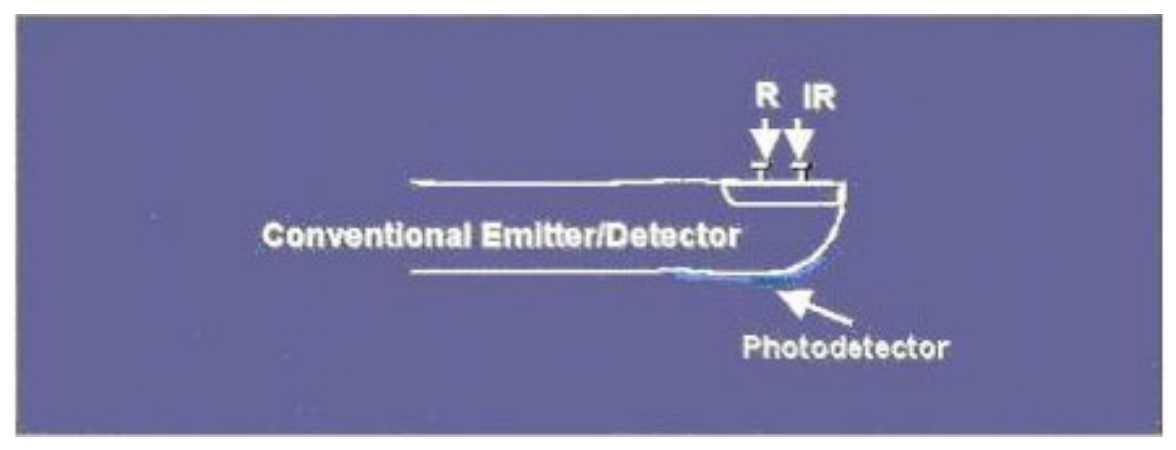

Fig.2.3 Transmission pulse oximetry (from [12])

The transmission method is most frequently used, and because it is more intuitive, the following explanation is given for it. The Red and IR radiation that passes through the measuring site arrives and is detected by the photo sensor. After being amplified and processed the ratio Red/IR is calculated and compared to a "look-up" table that converts the ratio to a $\mathrm{PO}_{2}$ value. Most manufacturers have their own look-up tables [12] 


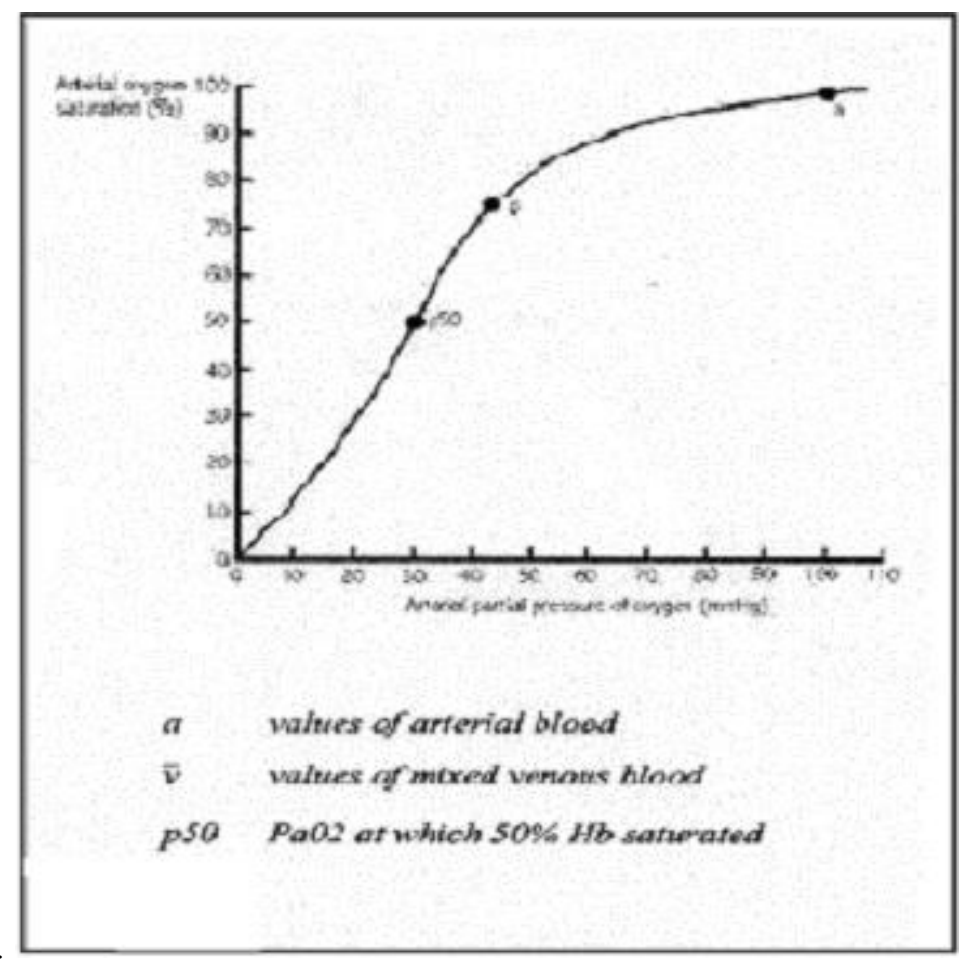

Fig.2.4 Relationship between Arterial Pressure of Oxygen and Arterial Oxygen Saturation. ([20], [22]-Ch.VII)

The relationship between the arterial pressure of oxygen and the oxygen saturation is described by the hemoglobin-oxygen dissociation curve (see fig.2.4). The sigmoid shape of this curve facilitates unloading of oxygen in the peripheral tissues where the $\mathrm{PaO}_{2}$ is low and oxygen is required for respiration. [20]

From a practical point of view, a pulse oximeter consists of:

- $\quad$ Peripheral probe (containing the photo emitters and detectors)

- $\quad$ DSP unit (which processes the signals obtained from the sensors)

- $\quad$ Display unit, showing a waveform, the oxygen saturation and the pulse rate.

- An audible pulse tone, the pitch of which is proportional to the oxygen saturation, useful when one cannot see the display (for instance the surgeon)

The DSP should be able to select out the absorbance of the pulsatile fraction of blood (due to arterial blood), from the constant absorbance (due to venous blood or capillary blood) and other tissue pigments.

Moving (AC) and non-moving (DC) absorbance ratio calculation:

$$
\mathbf{R}=\frac{\mathbf{A C}_{650} / \mathbf{D C}_{650}}{\mathbf{A C}_{900} / \mathbf{D C}_{900}}
$$

where $\mathrm{AC}_{650}$ is pulsatile absorbance value for visible red $(650 \mathrm{~nm})$;

$\mathrm{AC}_{900}-$ is similarly for infrared (IR);

$\mathrm{DC}_{900}$ - is continuous (non pulsatile) absorbance value for infrared (IR) $900 \mathrm{~nm}$.

$\mathrm{DC}_{650}-$ is similarly for visible red. 


\section{Advantages and details of this principle:}

For the purpose of minimizing the background noise, EMI (electromagnetic interference) and artifacts of different causes, the measurement can become sophisticated, and also more expensive. From [20], Introduction to "Practical Applications of Pulse Oximetry”, www.nda.ox.ac.uk/wfsa/html/u11/u1104 01.htm: “...Time division multiplexing, whereby the LED's are cycled: red on, then infrared on, then both off, many times per second, helps to eliminate the background noise. Quadrature division multiplexing is a further advance, in which the red and infrared signals are separated in phase rather then in time and recombined in phase later. In this way, an artifact due to motion or EMI, may be considerably reduced, since it will not be in the same phase of the two LED signals, once they are recombined. The saturation values are averaged out, over 5 to 20 seconds. The pulse rate is also calculated from the number of LED cycles between successive pulsatile signals and averaged out over a similar variable period of time, depending on the type of sensors. From the amount of light absorbed at each frequency, the DSP calculates the ratio..." The DSP is a Digital Signal Processor, a computing device dealing with the processing algorithms and calculations. A correlation of the result has to be done (fig.2.4). Within the pulse-oximeter memory, a series of oxygen saturation values are previously stored. Those values were obtained from experiments performed in which, human volunteers were given increasingly hypoxic mixtures of gases to breathe. The DSP compares the ratio of absorption at the two light wavelengths measured with these stored values, and then displays the oxygen saturation, digitally, as a percentage, and audibly (phonic) as a tone with varying pitch.

In the transmission mode, the percentage of the PPG (photo-plethysmogram) which is pulsatile can be seen to be about 5\% in the NIR (near infrared), which is about an order of magnitude bigger than in reflectance mode.([5], Results, p.7/2).

Reflectance method applies when the emitter and detector are situated on the same side of the measuring site. (Refer to Fig.1.1)

\subsection{Limitations of the pulse oximetry method}

There are factors, agents, or situations that may affect readings, limit precision, or limit the performance or application of pulse oximetry. Among those [20],[22]:

- $\quad$ Motion artifacts

- Abnormal hemoglobins (primarily carboxyhemoglobin [COHb] and methemoglobin [metHb])

- Intravascular dyes.

- $\quad$ Exposure of measuring probe (sensors) to ambient light during measurement.

- $\quad$ Low perfusion states.

- $\quad$ Nail polish or nail coverings with finger probe.

- $\quad$ Inability to quantitate the degree of hyperoxemia present.

- $\quad$ Inappropriate placement of the probe. 


\subsection{Conclusions}

After comparing Photo-Plethysmography and Opto-Spectroscopy (the sensing method of Pulse Oximetry) the decision was made in favor of Photo-Plethysmography, because of its simplicity and efficiency for the given conditions of this thesis (just live detection, without any concern on the user's health.)

A comparison was previously made between photo-plethysmography and some other known liveness detection methods, such as perspiration, temperature, skin resistivity, Doppler effect (sensing heart and respiratory movements with a microwave Doppler radar), RF (radio-frequency) bio-effects, etc.

\subsection{Pulse Oximetry examples}

One of the best examples of a pulse oximetry prototype reflectance sensor is found in [10], "Esophageal Pulse Oximetry Utilizing Reflectance PhotoPlethysmography".

Another interesting example of pulse oximetry sensor to mention, combining the use of multiple emitters and multi-path detection, is the PRO2 sensor from Imagyn: www.imagyn.com/criticalcare/pulse reflectance oximetry pgl.htm (the web site of Imagyn Medical Technologies from Haifa, Israel).

In contrast, the following example shows the use of one emitter (laser diode) and multi detectors, [4] "Sensor design of a new type reflectance pulse oximetry".

As for pulse oximeter monitors, in the first place are those manufactured by well-known companies, as Nellcor, Massimo, Biox, HP, Siemens, SurgiVet (for veterinarian purposes) and others.

A very simple, yet performing design has been done by Viktor Kremin, in Circuit Cellar \#155 (June 2003). He uses the pulse oximetry principle and a DSP made out with the help of a PSoC (Programmable System on-Chip device) from Cypress MicroSystems (CY26443) to build a "Remote Health Monitoring System" that received the first prize in the "Design Challenge - Cypress MicroSystems Contest", in 2002. 


\section{Chapter 3 PHOTO-SENSORS}

\subsection{Infrared Radiation (IR)}

\section{Electromagnetic (EM) spectrum.}

The Infrared region of the electromagnetic spectrum is generally defined as those wavelengths lying between the visible and microwave regions, the wavelengths between $7.5 \times 10^{-4} \mathrm{~mm}$ and approximately $1 \mathrm{~mm}$. Its importance arises from the fact that every material object emits, absorbs, transmits, and reflects infrared radiation in a characteristic manner.

Listed below are the approximate wavelength, frequency, and energy limits of the various regions of the electromagnetic spectrum.

\begin{tabular}{|c|c|c|c|}
\hline \multicolumn{1}{|c|}{} & Wavelength $(\mathbf{m})$ & Frequency (Hz) & Energy (J) \\
\hline Radio & $>1 \times 10^{-1}$ & $<3 \times 10^{9}$ & $<2 \times 10^{-24}$ \\
\hline Microwave & $1 \times 10^{-3}-1 \times 10^{-1}$ & $3 \times 10^{9}-3 \times 10^{11}$ & $2 \times 10^{-24}-2 \times 10^{-22}$ \\
\hline Infrared & $7 \times 10^{-7}-1 \times 10^{-3}$ & $3 \times 10^{11}-4 \times 10^{14}$ & $2 \times 10^{-22}-3 \times 10^{-19}$ \\
\hline Visual & $4 \times 10^{-7}-7 \times 10^{-7}$ & $4 \times 10^{14}-7.5 \times 10^{14}$ & $3 \times 10^{-19}-5 \times 10^{-19}$ \\
\hline UV & $1 \times 10^{-8}-4 \times 10^{-7}$ & $7.5 \times 10^{14}-3 \times 10^{16}$ & $5 \times 10^{-19}-2 \times 10^{-17}$ \\
\hline X-ray & $1 \times 10^{-11}-1 \times 10^{-8}$ & $3 \times 10^{16}-3 \times 10^{19}$ & $2 \times 10^{-17}-2 \times 10^{-14}$ \\
\hline Gamma-ray & $<1 \times 10^{-11}$ & $>3 \times 10^{19}$ & $>2 \times 10^{-14}$ \\
\hline
\end{tabular}

Fig.3.1 The electromagnetic spectrum - main regions (source [31])

The electromagnetic spectrum (EM) brings together all known types of radiation. IR Definition: IR is the portion of the EM spectrum lying between the visible and the microwave regions.

\subsection{Introduction to photo detection}

Photo detectors are semiconductor devices that convert incident light into an electric current. Essentially they may be broken into two categories:

1. Quantum detectors.

2. Thermal detectors.

Quantum detectors - convert the incoming radiation directly into charge carriers, in a semiconductor device, and process the resulting current with electronic circuitry. (photovoltaic cells, photo-diodes and phototransistors). 
Thermal detectors - absorb the radiant energy and operate by measuring the change in temperature with a thermometer. (thermistors, photo-resistors).(Not suitable for the applications needing high efficiency and fast response time).

The present design uses photo-PIN diodes, since they offer the best performance for detection of optical radiation, for the purposes of this thesis. The next paragraph gives reasons for choosing the photo PIN diode as photo-detector.

\subsection{Photodiodes, sensors with photodiodes}

Photodiodes are widely used for light intensity measurement, as well as light detection. Advantages over the thermal sensors (e.g. photo resistors):

- $\quad$ higher sensitivity

- $\quad$ faster response time

- $\quad$ smaller size

- $\quad$ better stability

- excellent linearity

The types of photodiodes are: $\mathrm{p}-\mathrm{n}$ photodiode, PIN photodiode, Avalanche photodiode and Shottky photodiode.

The normal PN junction having a window that permits the photon's beam to hit it.

In an ordinary pn junction the depletion layer is around a micron thick. As a result not much light is absorbed.

The PIN diode. (PIN $=p$, insulator, $n$.) The PIN diode idea is enhancing the junction surface (that is the surface where the energy transfer between photons and electrons takes place) the quantum efficiency is improved. (Number of electrons generated over total light incident).

Avalanche diodes (APD) use the effect of electron multiplication by avalanche in presence of a large reverse bias. (Sensitive but very noisy, we'll not be using those for our purpose).

When a metal is deposited on an n-type semiconductor, the resulting metal over semiconductor junction behaves similar to that of a pn junction diode. Schottky diodes are unipolar devices that are faster than bipolar. They are used in high frequency applications.

A photodiode is simply a diode, reverse-biased. When a photon is absorbed, an electron-hole pair is generated. From a circuit design stand point the photodiode's electrical behavior can be modeled with the small signal equivalent circuit model shown in Fig.3.2:

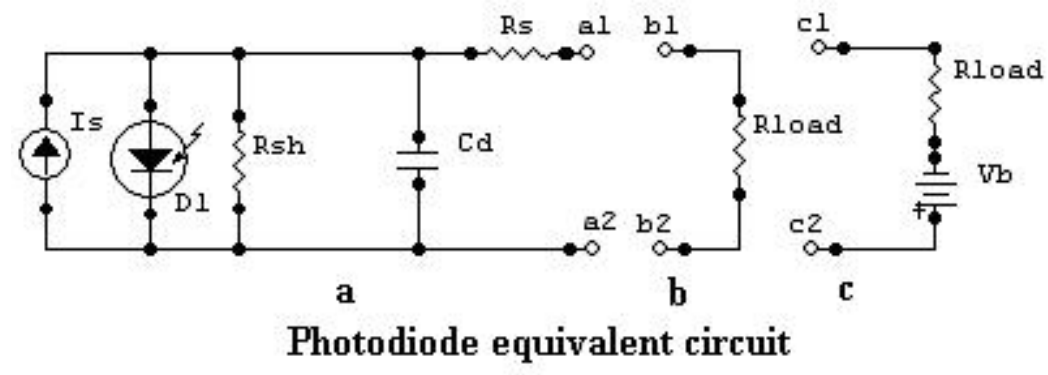

Fig.3.2 Photodiode equivalent circuit 
Parts a and $\mathrm{b}$ are combined for photovoltaic mode, parts a and $\mathrm{c}$ are combined for photoconductive mode of operation. ([31], page 434).

The generated charges are measured between the terminals directly (photovoltaic mode of operation) or by reading the reverse bias current (photoconductive mode of operation). In Figure 3.2 the current generator produces a current Is proportional to the absorbed light. D1 is an ideal diode (a p-n junction with a forward current Id). The internal impedance of the cell is modeled by the series and parallel terms, Rs (series resistor), Rsh (shunt resistor, or parallel), Cd (internal capacitance of the junction). In the photovoltaic mode the load resistor Rload is connected parallel to the output. In the photoconductive mode a source of external bias voltage $\mathrm{Vb}$ is connected to the cell. The current Is is proportional to the light irradiance falling on the cell (Io) and to the area of the cell, A:

$$
\text { Is }=(\eta \operatorname{IoAq} \lambda) /(\mathrm{hc}) ; \quad(3.3)
$$

Where: $\eta=$ quantum efficiency of the absorption (usually in the range of 0.6 to 0.9 )

$$
\begin{aligned}
& \mathrm{A}=\text { area of the cell } \\
& \text { Io }=\text { incident irradiance }\left[\mathrm{W} / \mathrm{m}^{2}\right] \\
& \mathrm{q}=\text { the electronic charge }\left(1.69 \times 10^{-19}[\mathrm{C}]\right) \\
& \mathrm{h}=\text { Planck's constant }\left(6.626 \times 10^{-34} \mathrm{~J} / \mathrm{s}\right) \\
& \mathrm{c}=\text { speed of light }
\end{aligned}
$$

Note: The capacitance $C d$ should be taken into account if the cell is operated at a relatively high optical modulation frequency. So for low frequencies it can be assumed that Icd=0. (For more details, see [31], pages 434 to 441).

Figures 3.3 and 3.4 are showing basic detection / amplifier circuit implementations for the photovoltaic and photoconductive modes of operation.

\subsubsection{Photovoltaic mode}

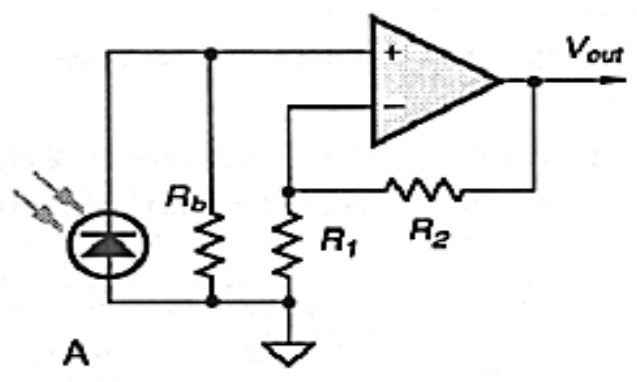

\section{Fig.3.3 Connection of a photodiode in a photovoltaic mode}

In the photovoltaic mode, the diode is attached to a virtual ground preamplifier as shown in fig.3.3, and the arrival of photons cause the generation of a voltage that is amplified by the op-amp. The primary feature of this approach is that there is no DC bias across the diode aside from the thermally generated currents. This configuration does suffer from slower response, because the charge generated must charge the capacitance of the diode, causing a RC-delay. 


\subsubsection{Photoconductive mode}

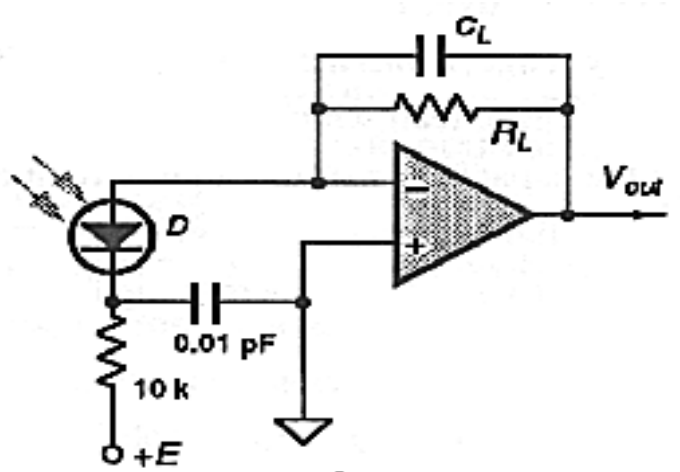

A

\section{Fig.3.4 Photoconductive operating mode}

In the photoconductive mode, fig.3.4, the diode is biased, and the current flowing across the diode is converted to a voltage (by a resistor) and amplified. The primary advantage of this approach is that the applied bias, decreases the effective capacitance of the diode (by widening the depletion region), and allows for faster response. Unfortunately, the DC bias also causes some leakage current, so detection of very small signals is compromised.

\subsubsection{Conclusion}

From the previous two modes of operation the photovoltaic mode is suitable for the work of this project because, despite a slower response (that doesn't affect in this case), it allows the detection of smaller signals.

\subsection{Other photo-detectors}

- photo-transistors

- $\quad$ photo-Darlington

- $\quad$ OPT 301

Among the phototransistors there are the bipolar phototransistor and the photoDarlington transistor. Here the photocurrent is multiplied by the Beta of the transistor, providing gain. However, the high speed performances is degraded because of the collector to base capacitance. The OPT 301 consists of a photo-diode and an Op Amp embedded in the same chip. 


\subsection{Comparison of response times for different photodetectors}

1. Photodiode: $10 \mathrm{nS}$.

2. Bipolar phototransistor: $5 \mathrm{uS}$.

3. Reflective photo sensors: 20uS. (e.g. CNB 1303 - Panasonic)

4. Photo-Darlington: $50 \mathrm{uS}$.

5. OPT 301: 90uS. (This is a device from Burr-Brown, containing an operational amplifier, which will be used further in the design).

\subsection{Custom made sensors for special applications}

There are, commercially available sensors built for a lot of purposes and applications, but sometimes (as is this case) one needs to build a unique model of sensor, targeted especially for a specific application.

This project needs a reflectance photo sensor to detect photo-plethysmography out of the human palm. Among the reflectance sensors readily available, the following "Reflective Object Sensors" or "Retro Sensors" were utilized during the research:

1. OPB 607A (from OPTEK, non-focused, with photo-Darlington)

2. QRB 1133 and QRD 1114 (from Fairchild, with phototransistors, non-focused)

3. CNB 1303 (from Panasonic, a GaAs IR LED integrated with a high sensitivity Silicon phototransistor in single resin package).

Those sensors didn't give enough satisfaction when targeted for detecting plethysmography out of the palm so building an original reflectance sensor was considered the best strategy to satisfy all needed constraints for this given project.

\section{Constraints:}

1. The sensor has to work through reflectance. It has to be placed under the palm, somewhere on the platen of the hand geometry scanner, such that it will not interfere with the IR beam coming from above (for geometry reading).

2. The price of the whole unit should be very inexpensive, in order to be easily embedded with the rest of the scanner. This is the reason the most common, inexpensive photo detectors and Ics were chosen for this design.

3. The volume for the liveness detector should be very small; it has to be hidden into the original volume, such that no other modifications should be made to the original design of the case.

Note1: At the level of prototyping the third constraint wouldn't give much concern because after everything works and gets the OK from the scanner supplier, a single chip containing the whole circuit can be designed.

Note2: There are no reflectance sensors for plethysmography or pulse oximetry, commercially available, unless you don't buy the whole pulse oximetry monitor! 


\subsection{Designing sensors}

Time was spent during the research in both directions, spoofing the device and detecting liveness in the palm. Spoof procedures will be covered later in the project.

For liveness a choice was made for optical quantum sensors. To see which one would work best, several types of photo-sensors available were tested, several wavelengths were used (NIR).

\subsubsection{Sensitivity and Peak-wavelength}

The sensitivity of the photo-detector is a function of wavelength, so to maximize the power efficiency the emission wavelength of the optical source should be spectrally matched to the photodiode. Silicon photodiodes that are used in low-cost applications (as the one in this project) have their peak spectral efficiency in the near infrared region (NIR). Fig.3.5 shows the normalized spectral sensitivity of a typical silicon photodiode (data taken from Panasonic's PNZ334 device) and shows how it is spectrally matched to GaAs or Ga AlAs infrared emitting diodes (IRED) (e.g. LN77L - Panasonic, QED423 Fairchild Semicond.) There is a linear relationship between the photocurrent $i_{s}$ and the irradiance, $E_{\mathrm{e}}$ (a measure of the intensity of the incident light $\left[\mathrm{W} / \mathrm{m}^{2}\right]$ ) - see the expression (3.3) page 22 .

\section{Spectral sensitivity characteristics}

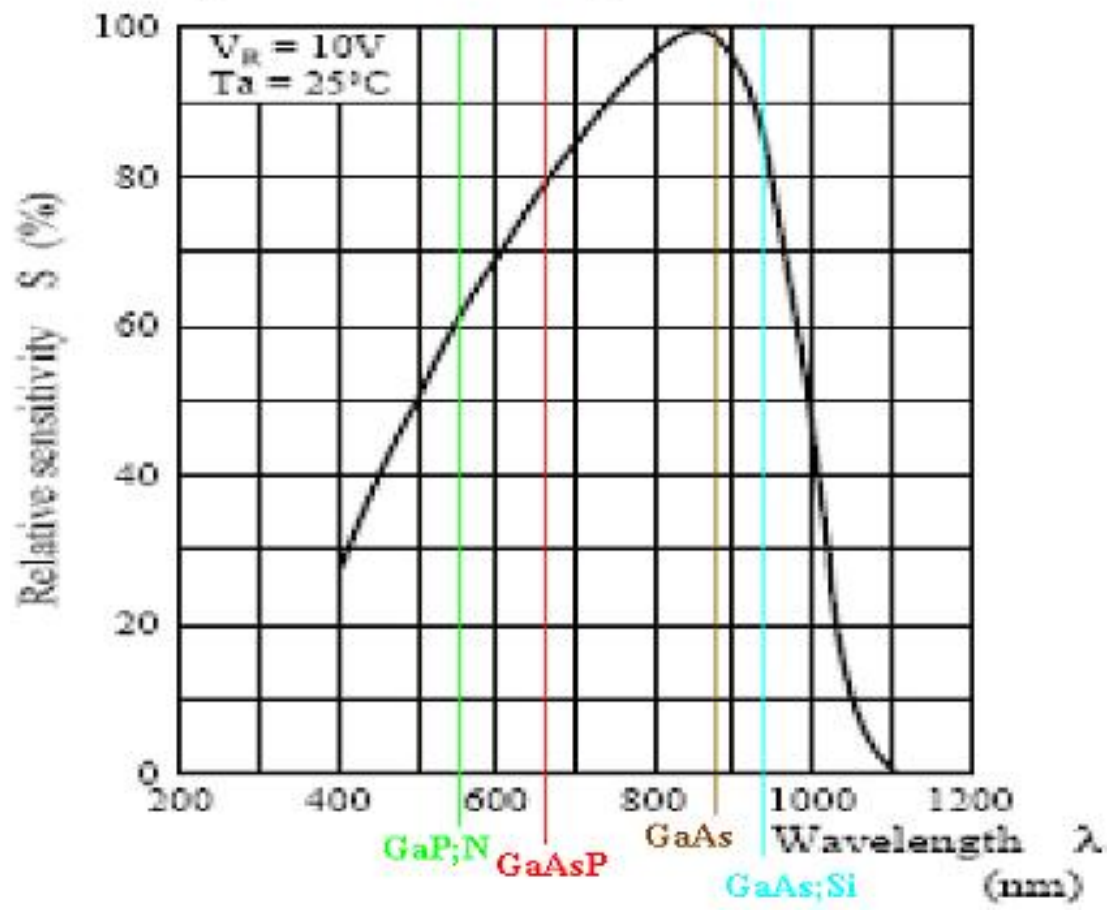

Fig.3.5 Relative spectral sensitivity of PNZ334 silicon photodiode and the peak wavelength emissions of several types of LEDs. 
(adapted from the data sheet, see appendix A.A2)

The sensor physical implementation started by cutting in two pieces the opto interrupter switch H22B1 from Fairchild Semiconductors.
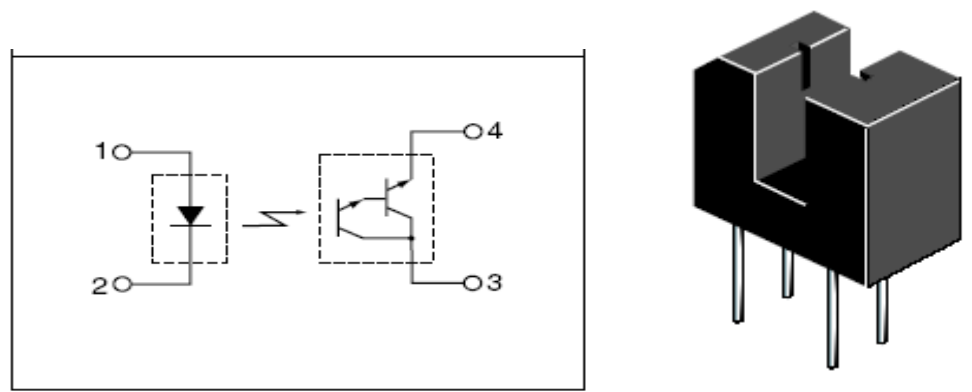

Fig.3.6 Opto-Interrupter Switch from Fairchild Semiconductor, H22B1

The detector from fig.3.6 is a photo-Darlington. The Emitter is an IRED, with a peak wavelength close to $900 \mathrm{~nm}$ (NIR). (From the data sheet, www.digikey.com )

When a fingertip was interposed between the emitter and the detector, the response of the circuit was a periodical voltage (see fig.3.7). The schematic used was:
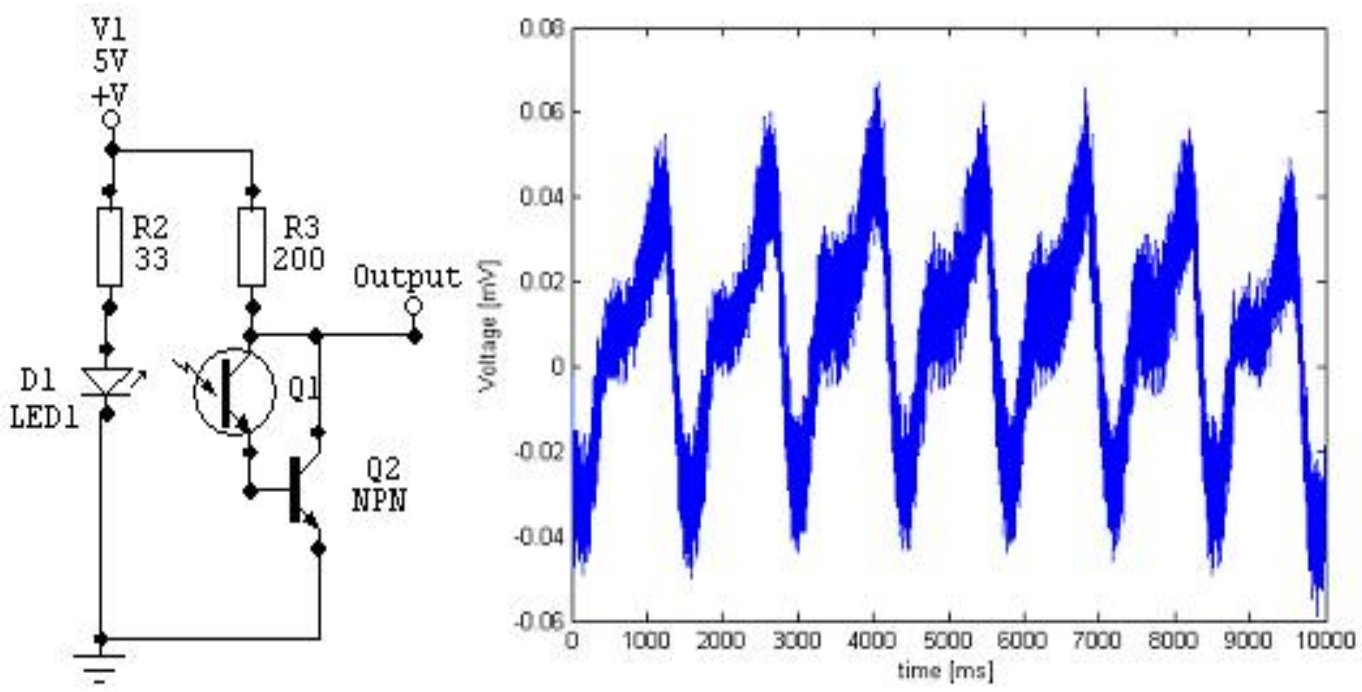

Fig.3.7 Schematic for a Trans-impedance Amplifier with photo-Darlington and its output signal response

The idea was to check how this kind of circuit works in $\mathrm{DC}$, at $1 \mathrm{KHz}(5 \mathrm{~V}$ sine wave), at $10 \mathrm{KHz}$, at $100 \mathrm{KHz}$.

The conditions checked were:

- $\quad$ in the presence of day light, fluorescent light.

- $\quad$ in the absence of light.

- $\quad$ with different kind of fingers: human, latex and play-doh. 


\subsubsection{Results}

1. The best detection took place in the absence of ambient light, a signal as in the figure 3.7 was obtained (acquired as an Excel coma delimited spreadsheet file on a floppy disk, from an oscilloscope Tektronics TDS 3052B and converted into image with the help of Matlab 6.5 software (import, plot)):

2. In the presence of light, the signal was satisfactory, but corrupted by noise if the external light was the fluorescent light. Applying the FFT transform, from the Math Block of the scope, the moving cursor found that the highest noise harmonics interfering with our signal were $60 \mathrm{~Hz}, 120 \mathrm{~Hz}, 180 \mathrm{~Hz}$, and so on, coming out of the fluorescent lamp. For the rest of the harmonics, responsible is EMI coming from the cables and apparatus in the Laboratory.

3. If instead of the DC supply, for the IRED is supplied frequency (periodical, sine wave signal) the following results occur:

- $\quad$ at a range $100 \mathrm{~Hz}$ to $1 \mathrm{KHz}$, everything seem to work $\mathrm{OK}$, a sine wave was received at the detector side.

- above $1 \mathrm{KHz}$, the amplitude and shape of the sine wave, at the detector side, degrades a lot. At $100 \mathrm{KHz}$, the attenuation is huge.

\subsection{Examples of transmission sensors.}

\subsubsection{Transmission Sensor 1}

In the beginning of the research, transmission sensors were built, because the transmission method is definitely more simple and intuitive, and not so prone to artifacts, noise and scattering.

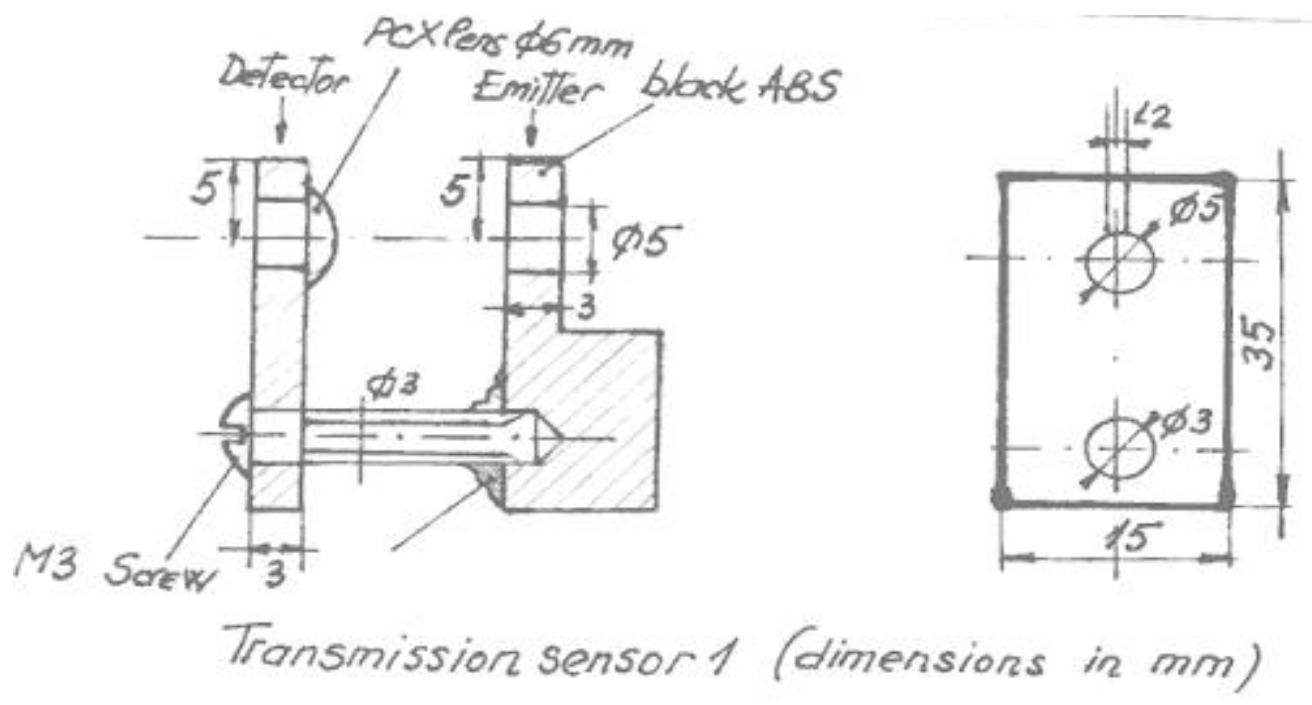

Fig.3.8 Technical drawing with the Transmission Sensor 1 


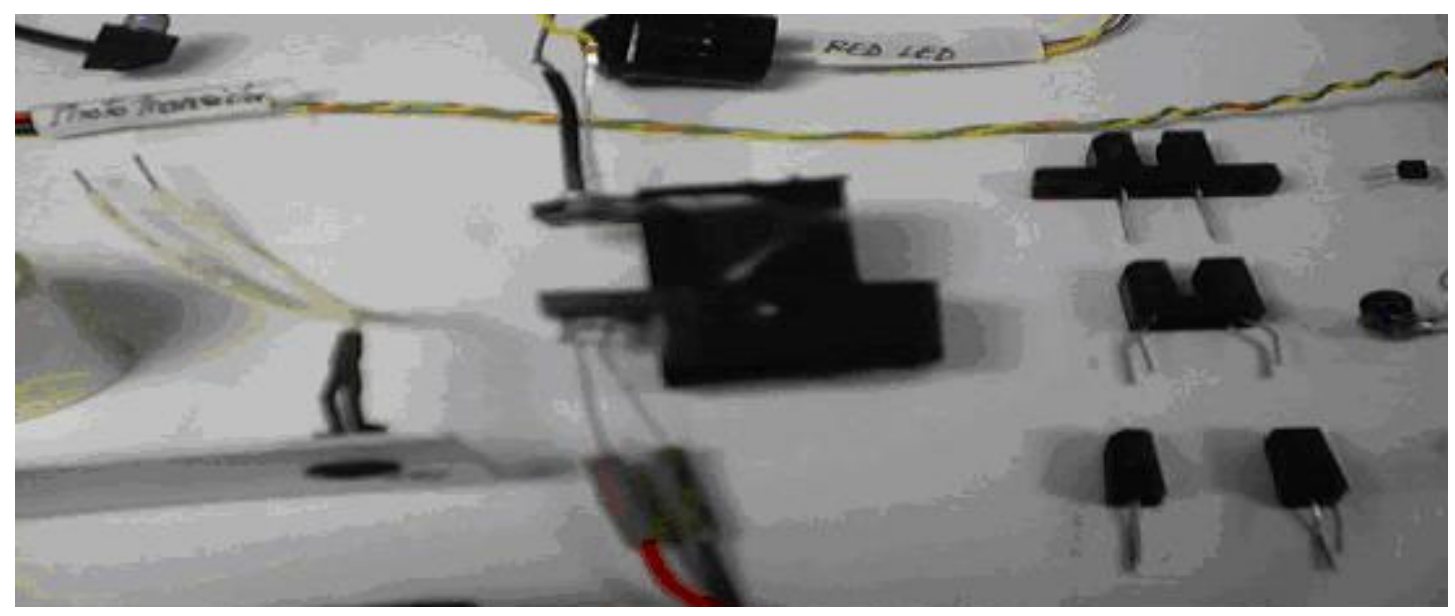

Fig.3.9 Photograph with the ABS plastic, Transmission Sensor 1, in center.

\subsubsection{Transmission Sensor 2}

The best results were obtained with the black plastic (two pieces of ABS plastic held together with a screw) and with a "U" shaped piece of aluminum as shown in the center of fig $3.10 \mathrm{~B}$
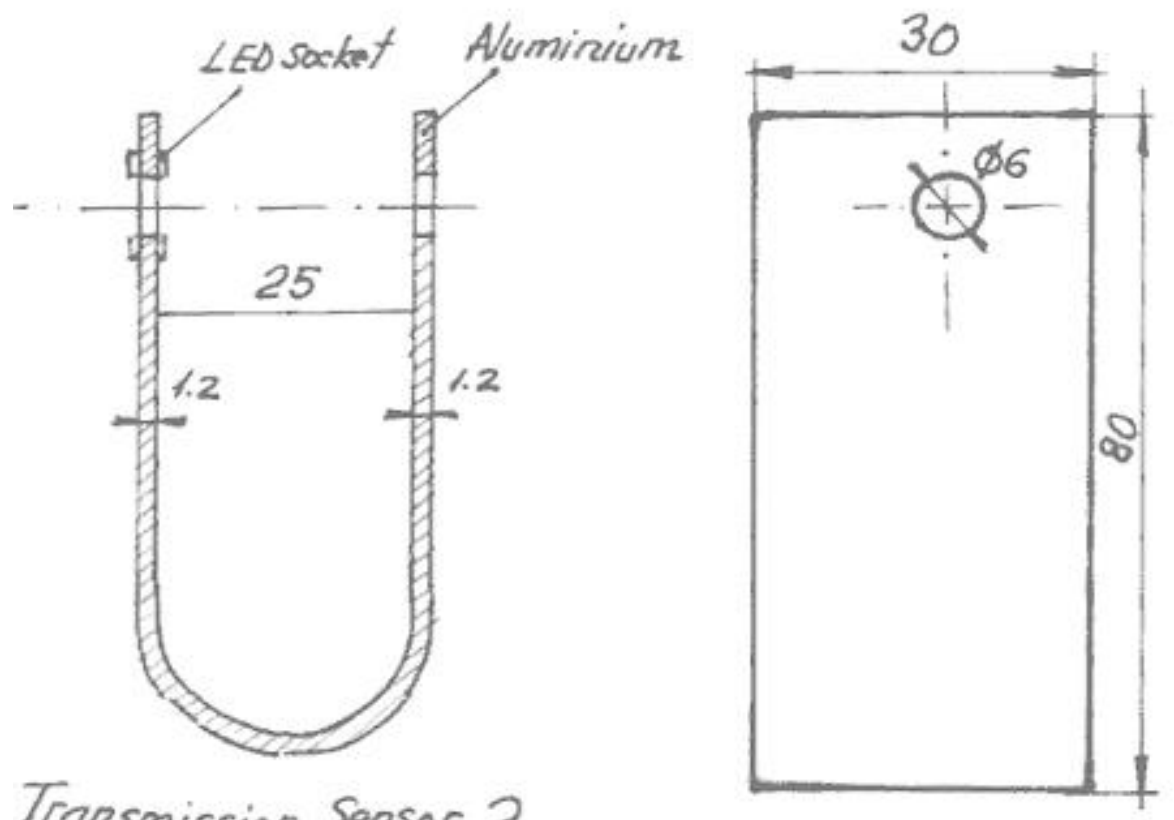

\section{Transmission Sensor 2}

Fig.3.10 A Technical drawing for Transmission Sensor 2 


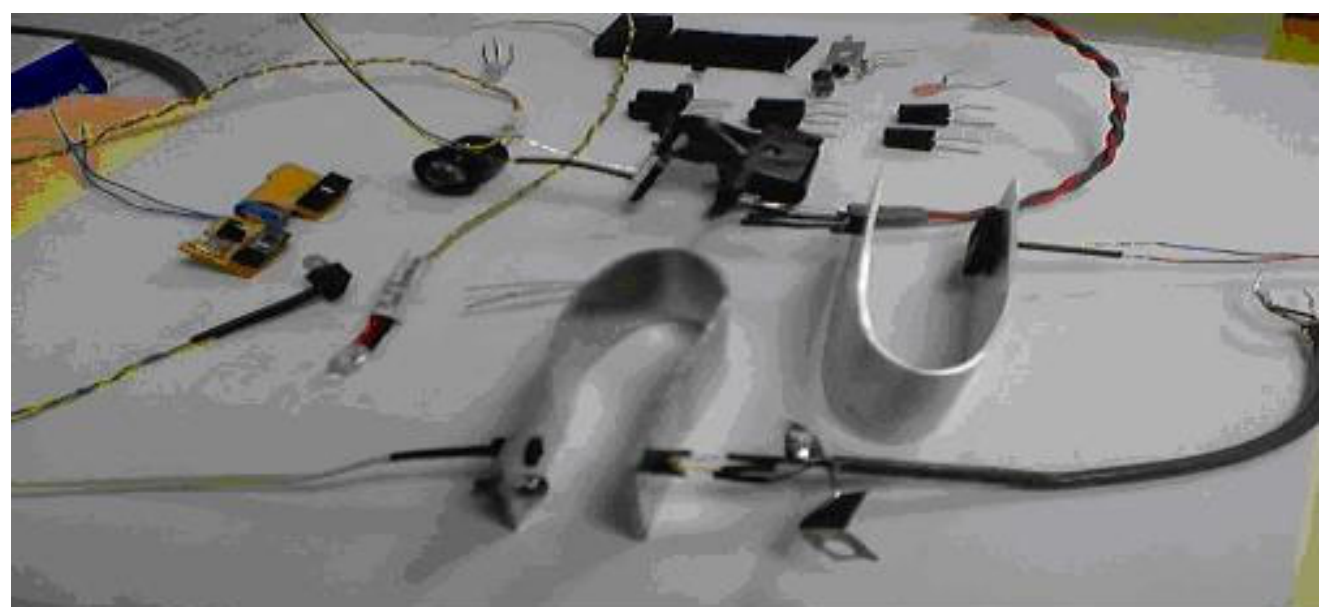

Fig.3.10 B Transmission sensor 2

The Transmission Sensor 2 is made of a thin $(1.5 \mathrm{~mm})$ Aluminum plate band bent in a "U" shape, such that the sensors will come one in front of the other, as in the picture.

\subsection{Example of Reflectance Sensors}

\subsubsection{Single Pair Reflectance Sensor}

Building reflectance sensors requires trial and error to fully understand their characteristics. Then a technical drawing with materials and dimensions has to be produced. A skilled technician has to use professional tools to cut, adjust, drill holes and glue the parts according to the design.

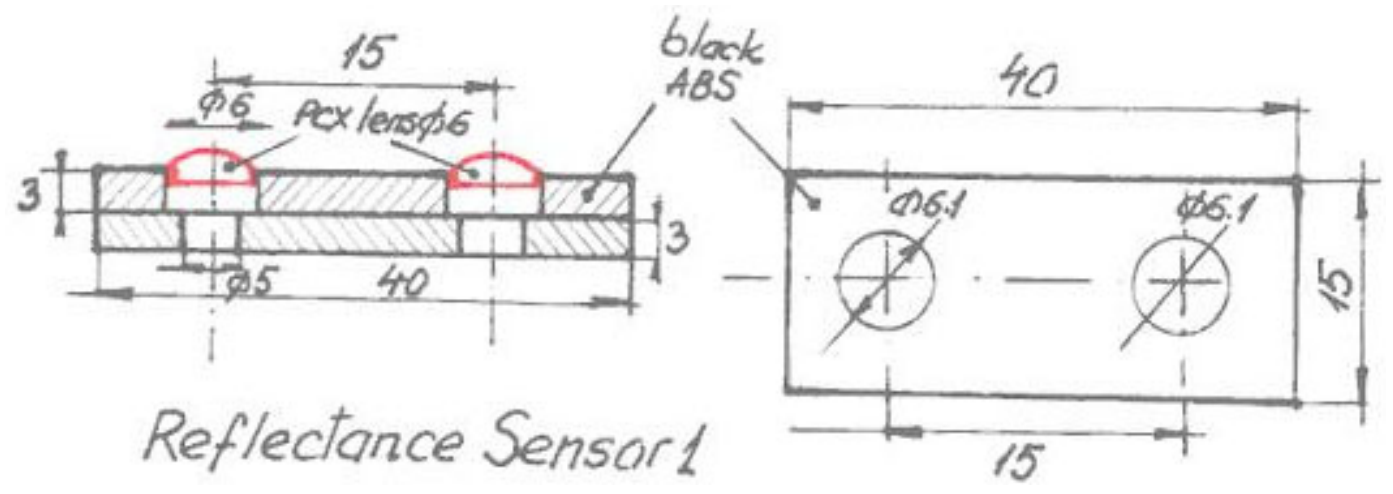

Fig.3.11 Single Pair Reflectance Sensor

As one can notice in fig.3.11 and 3.12, the basic material used was black ABS, easy to manipulate (cutting and drilling) opaque and non-reflective. As glue was used the hot glue gun, epoxy patch, epoxy gel, super-glue and plastic adhesive. At all times dimensions and alignments should be exact, otherwise the performance will degrade drastically. 


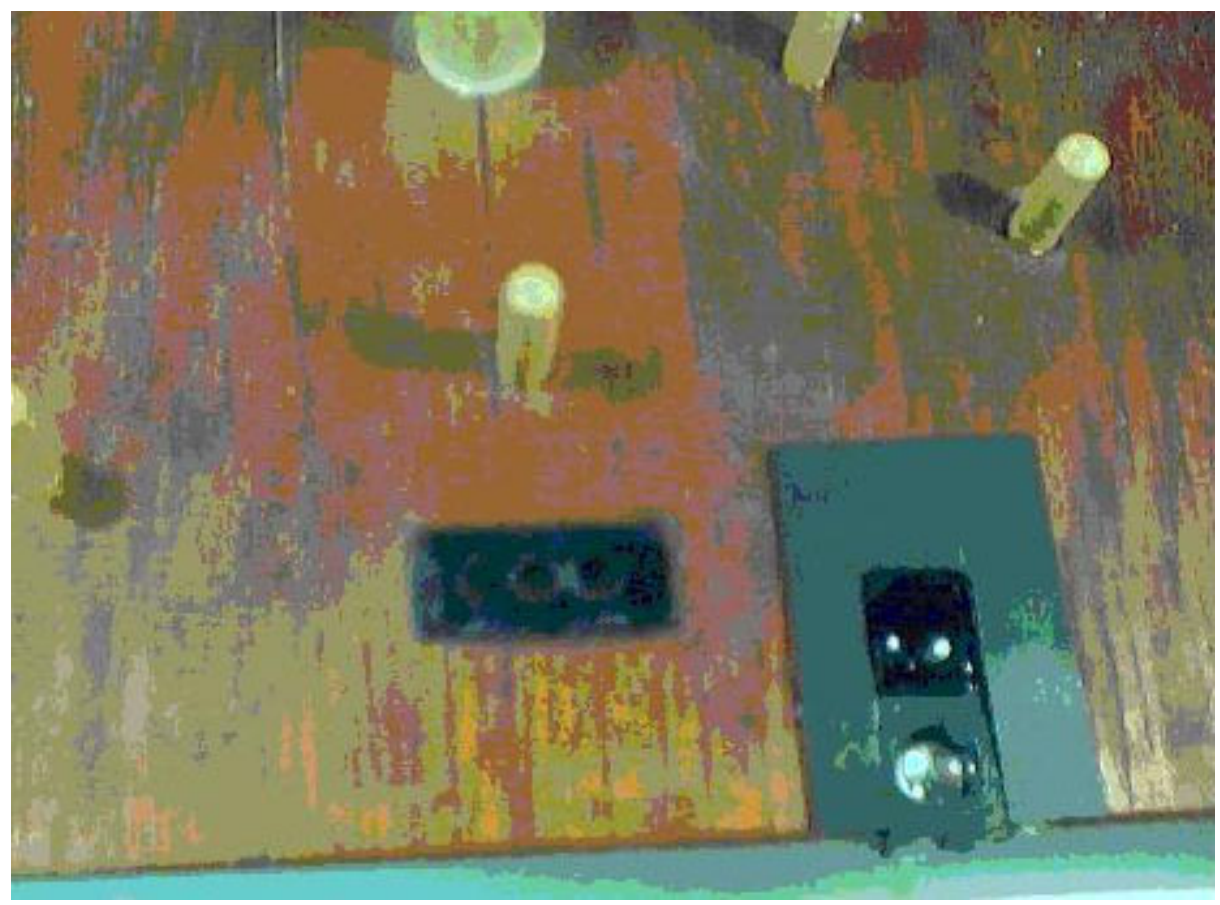

Fig.3.12 Single pair reflectance sensor embedded in a wooden platen

\subsubsection{Special Multi-emitter Sensor}

During the research, a sensor containing 4 emitters surrounding a detector in the middle was built. (See the technical drawing).

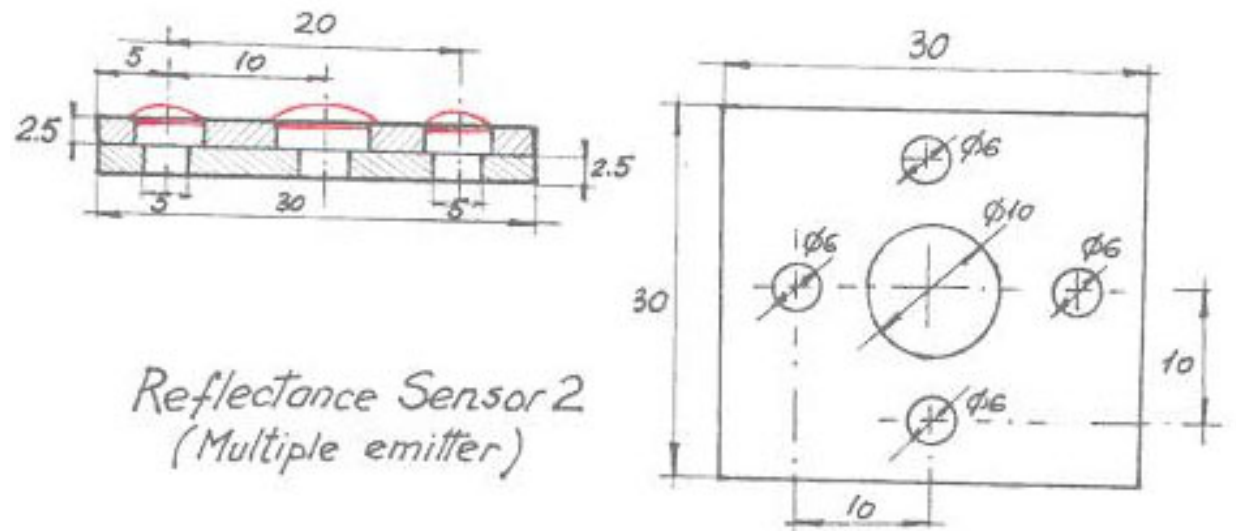

Fig.3.13 Multi-emitter Sensor (Reflectance Sensor 2)

Notice four emitters surrounding one detector in the middle 

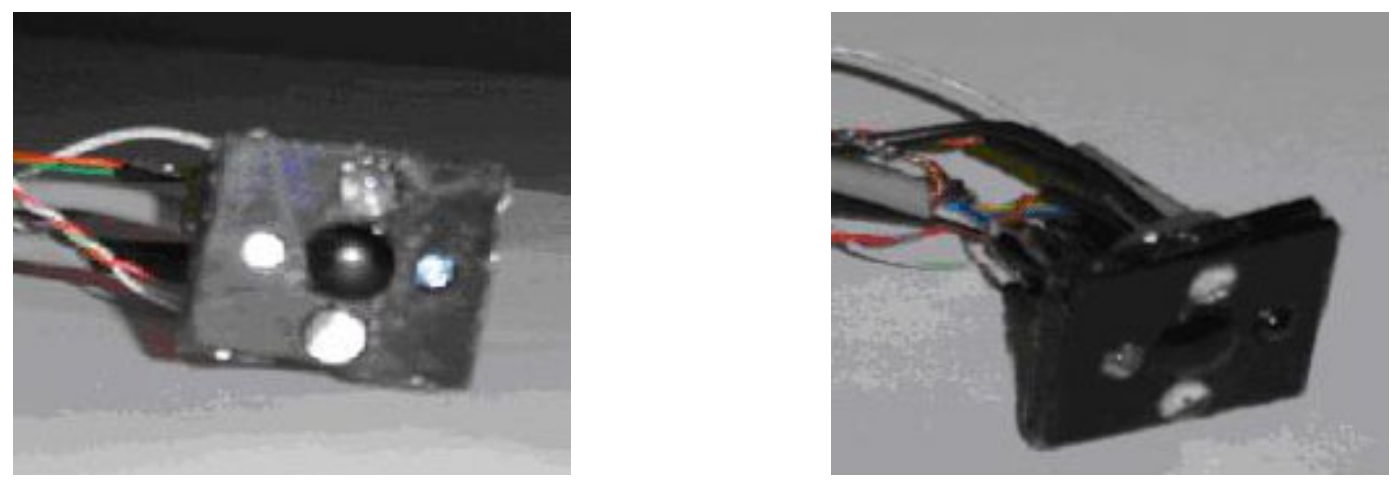

Fig.3.14 Picture with Reflectance Sensor 2 (multiple emitters)

The emitters used for this sensor were: QED 423 from Fairchild Semiconductor.

$\left(\mathrm{I}_{\mathrm{E}}=20 \mathrm{~mW} / \mathrm{sr}\right.$ at $\left.\mathrm{I}_{\mathrm{F}}=100 \mathrm{~mA}\right)$

The results were not spectacular. The following problems occur:

- In the one emitter-one detector approach, a $20 \mathrm{~mW}$ infrared LED (IR ED) was used. These LEDs are powerful but their power consumption is high, too. To achieve the maximum radiant intensity $\mathrm{I}_{\mathrm{E}}$ (maximum IR light), that is $20 \mathrm{~mW}$, according to the data sheet, the current consumption is $100 \mathrm{~mA}$. So, just one emitter sinks $100 \mathrm{~mA}$, twice the current consumption of Analog, Logic and Decisional Blocks, altogether! When building the sensor containing four emitters of this kind, just the current consumed by the emitters will be around $0.5 \mathrm{~A}$, making it of little value to build, no matter how high the signal strength will get.

- $\quad$ The second drawback of the multi emitter sensor is the volume that is four times greater than the one-to-one variant.

\subsection{Sensor Optics}

As stated in the future work part (Chapter 9), the Optics are very important in order to enhance the photo-plethysmographic signal arriving at the input of the first amplifier stage. More magnitude of the signal, better signal to noise ratio, thus less effort to process and extract the R-R interval.

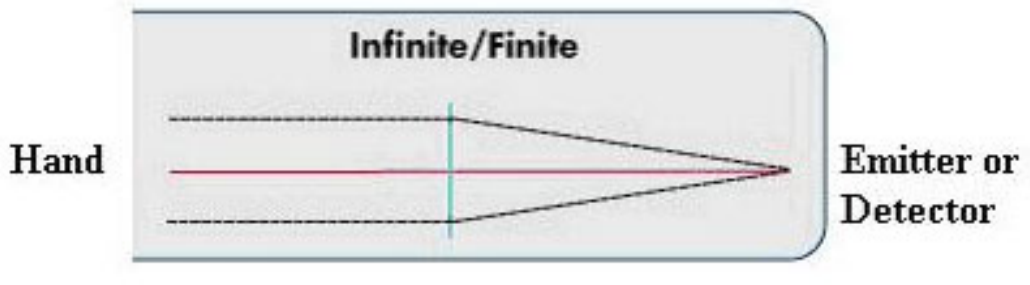

Fig.3.15 PCX (plano-convex) lens (from [14])

To be able to collimate, the case of infinite to finite conjugates was assumed (see fig.3.15). PCX (plano-convex) lenses were used to collimate the emitter and the detector beams. A coating (acting like a filter) may be added on both sides of the lenses. 
The lenses used for this project are made of $\mathrm{CaF}_{2}$ (Calcium Fluoride).

$\mathrm{CaF} 2$ can be used in the UV through IR, due to its high transmission from $250 \mathrm{~nm}$ to $7 \mathrm{um}$. Those lenses can be used without an antireflective coating, because of their low refractive index. The wizard proposed by the Edmund Optics Catalog [14] for a non-imaging system to collimate light was used to find the appropriate lens and coating.

The lenses ordered have the following specifications (part of the specs)[14]:

\begin{tabular}{|l|l|l|l|l|}
\hline Diameter & Effective FL & Glass Type & Stock number & Price \\
\hline $6 \mathrm{~mm}$ & $6 \mathrm{~mm}$ & SF11 & F45-077 & $\$ 18.00$ \\
\hline $9 \mathrm{~mm}$ & $9 \mathrm{~mm}$ & SF11 & F45-081 & $\$ 18.10$ \\
\hline
\end{tabular}

The recommended coating:

AR, code 146, range: NIRII - from 700 to $1550 \mathrm{~nm}$. Material used: LaKN22.

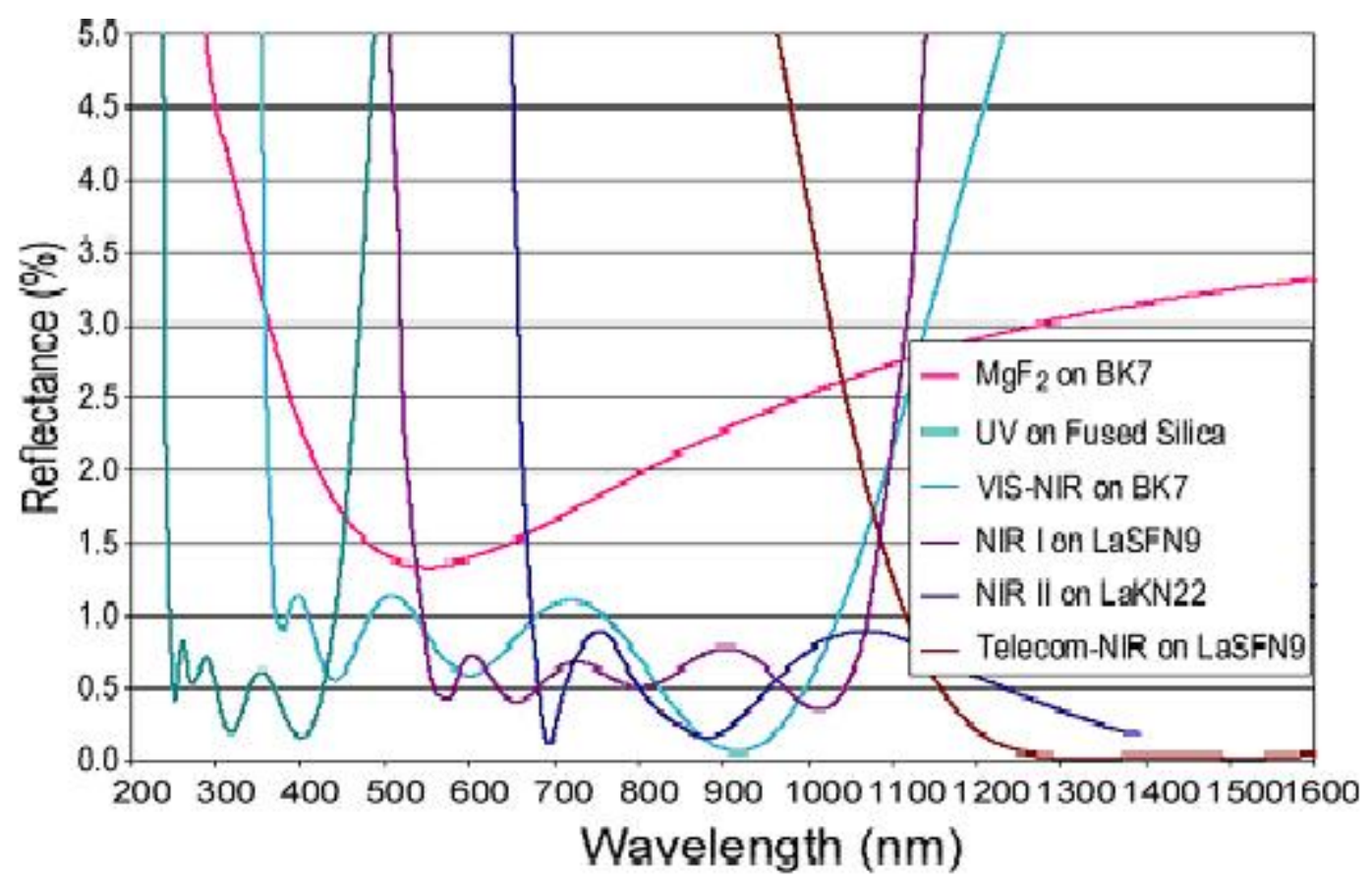

Fig.3.16 The figure explains how the coating may be chosen, and what is the expected range of wavelengths transmitted and stopped.(from [14]) 


\section{Chapter 4 PREAMPLIFIERS FOR OPTICAL SENSORS}

\subsection{Introduction on preamplifiers.}

At the sensor interface, preamplifiers are used to buffer and gain the sensor output. Typically, the preamplifier converts the received photocurrent into a voltage signal. The preamplifier plays a crucial role in determining many aspects of the overall performance of the whole circuit including speed, sensitivity and dynamic range. Optical preamplifiers are based on either a termination resistor or a trans-impedance amplifier. In the first approach, the choice of the load resistance affects both the frequency response and the noise performance of the preamplifier. The second approach (trans-impedance amplifier) was used for this project because it avoids the lack of sensitivity, low noise performances and dynamic range problems associated with the termination resistor approach.

Fig.4.1 shows the basic configuration and gain expression for the trans-impedance amplifier.

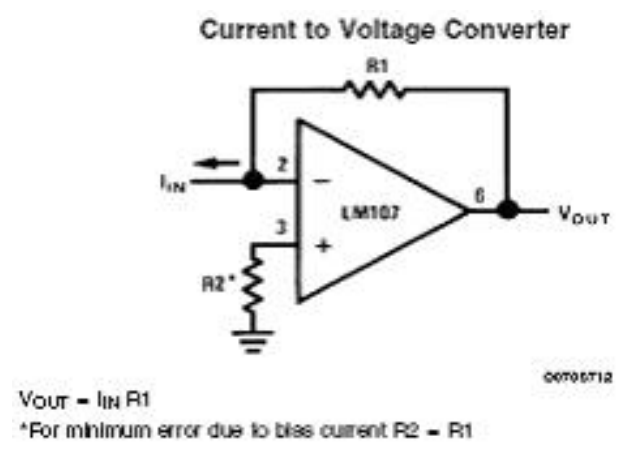

Fig.4.1 Trans-impedance Amplifier (Current Controlled Voltage Source)

In the trans-impedance approach the resistor can be made large because the negative feedback reduces the effective resistance seen by the photodiode by a factor of $1+$ Gain, where Gain is the open loop voltage gain of the amplifier. As a result the bandwidth can be matched to that of the signal, eliminating the need of equalization. In addition, the thermal noise contribution of the feedback resistor is minimized.

The closed-loop trans-impedance gain is defined as the ratio:

$\mathrm{V}_{\text {out }} / \mathrm{i}_{\text {in }}=-[$ Gain $/(1+$ Gain $)] \mathrm{R}_{1}=\sim-\mathrm{R}_{1}$;

Where: $\mathrm{V}_{\text {out }}$ and $\mathrm{i}_{\text {in }}$ are respectively the output voltage and the input current

Gain is the open loop voltage gain of the amplifier.

$\mathrm{R}_{1}=$ the feedback resistor (see figure 4.1)

The point of this calculus is that for large open loop gains, the trans-impedance gain is simply the feedback resistance with a minus sign, due to the fact that the inverting input of the op amp was used. 


\subsection{Circuits used as Amplifiers for sensors}

\subsubsection{For the normal photodiode and PIN photodiodes}

The circuit used is showed in fig.4.2:

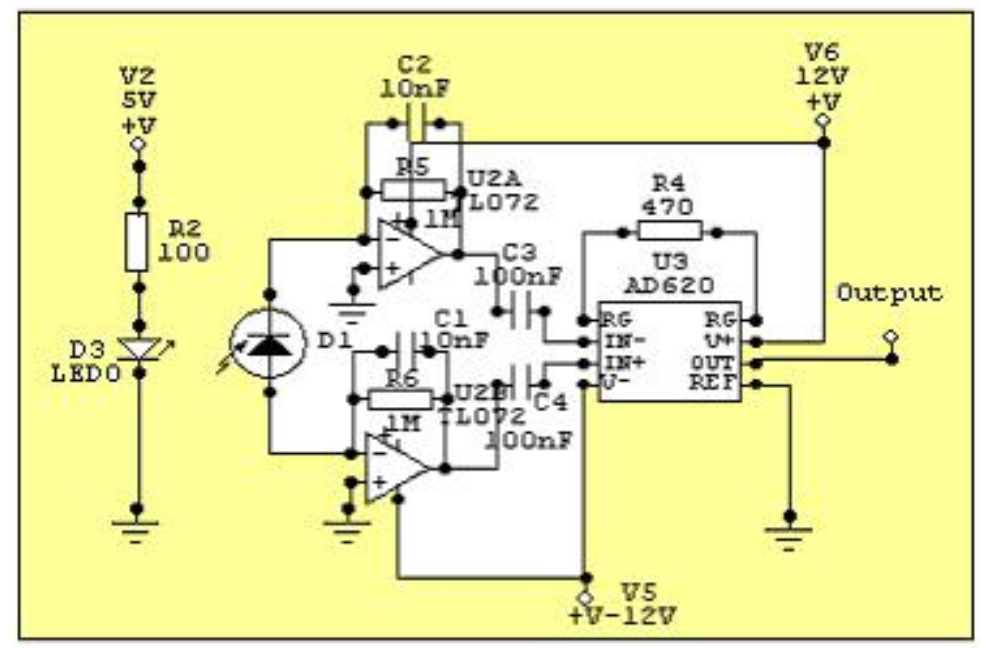

Fig.4.2 Op Amp circuit used for PIN photo diodes

The Emitter IRED, D3, is biased for its maximum current that will provide the maximum IR optical power output. D3 is a GaAlAs IRLED, high power and high efficiency, from Panasonic. D1 is a PIN photodiode, PNZ 334, from Panasonic. (see Appendix A for its data sheet) They have both a peak wavelength of $860 \mathrm{~nm}$ (NIR). U2, A and B is the IC TL072, a dual Op Amp, with JFET inputs build for very low noise. Due to JFET inputs, the input impedances of both amplifiers are very high, enhancing the capture of weak signals. (signals having a bad signal to noise ratio). U2A section amplifies the cathode current, U2B section amplifies the anode current, both are connected as trans impedance (or trans resistance amplifiers) with a gain (in ohms) of 1000 given by RF (1M).

Explanation:

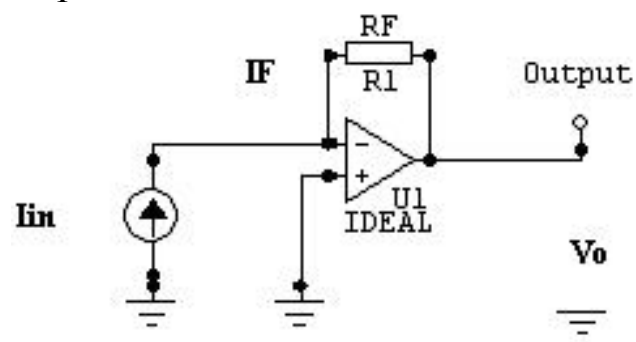

Fig.4.3 Transimpedance Amplifier

$\mathrm{IF}=\operatorname{Iin}$; (because of the virtual ground) (1)

$\mathrm{Vo}=-\mathrm{IF} * \mathrm{RF} ;(\mathrm{Ohm}$ law) (2)

From (1) and (2) $=>$ rm=Vo/Iin=RF; (Q.E.D.) 
The transimpedance amplifier stage and the voltage amplifier stage are coupled through a $100 \mathrm{nF}$ capacitor, which leaves the frequency practically unaltered but stops the DC component from propagating to the next stage.

As for the second stage, the voltage amplifier, a precision instrumentation amplifier was used, for accurate low noise, differential signal acquisition, INA126, from Burr-Brown.

As the fig.4.4 shows, the gain of this instrumentation amplifier is given by the data sheet: $\mathbf{G}=\mathbf{5}+\mathbf{8 0 K} / \mathbf{R G}$; where $\mathrm{G}$ is the gain, $\mathrm{RG}$ is the resistance between the pins 1 and 8 of the IC. With a RG $=470$ as in the circuit, a gain of 175 results. This gain will be enough to bring the "ECG like" pulsatile signal, from tens of milivolts to volts.

The calculation of the output voltage magnitude of the analog block starts assuming Is (the current generated by the photodiode, proportional with absorbed light, figure3.3, and (3.3) formula, page 17), for the maximum optical power of the Infrared Emitting LED is Is $=10 \mu \mathrm{A}$. With a feedback resistor of $1 \mathrm{M}$, the trans-impedance amplifier will output a voltage in the range of $10 \mu \mathrm{Ax} 1 \mathrm{M}=10 \mathrm{mV}$; at the output of the instrumentation amplifier (the voltage amplifier) the magnitude of the signal will be in the range of volts:

$10 \mathrm{mVx} 175=1.75 \mathrm{~V}$;

Magnitudes in the range of volts are needed to be able to further prepare the shape of this signal in order to be finally processed by the Decision Block.

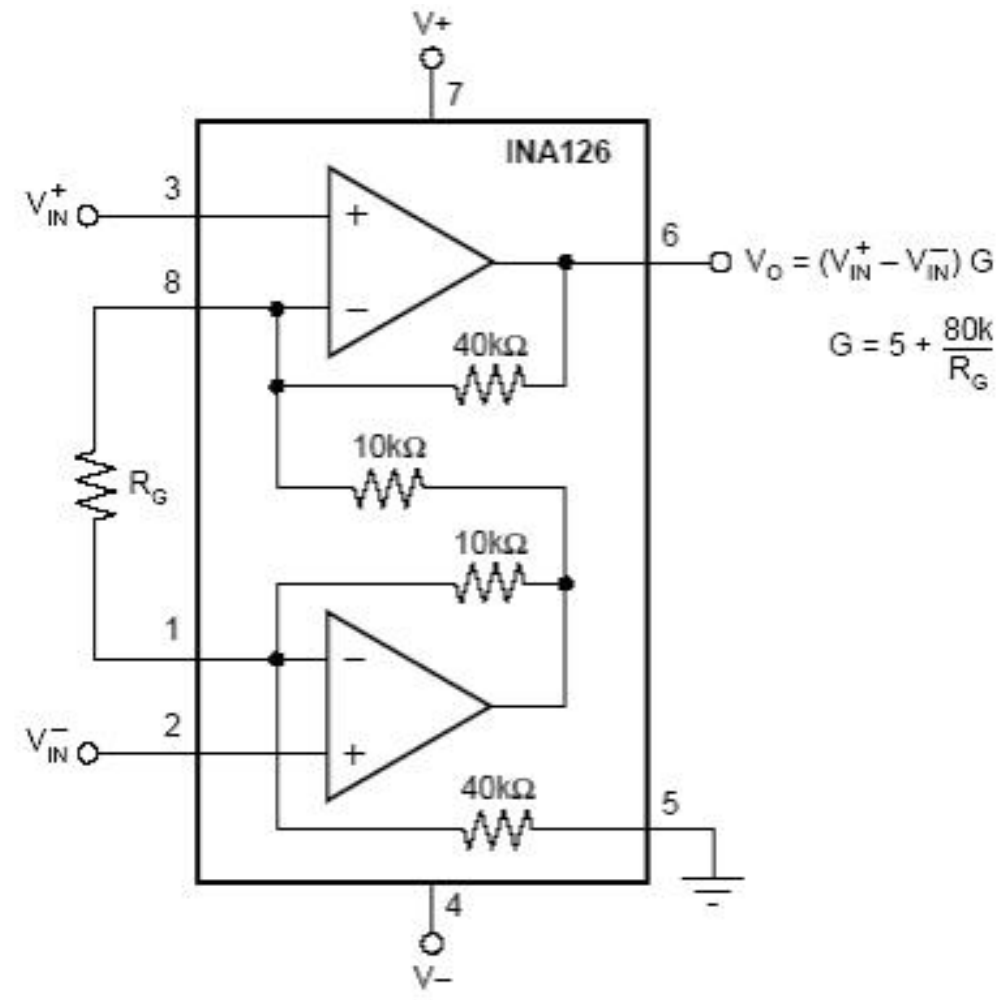

Fig.4.4 Second stage (Voltage Amplifier) with INA 126

(Figure copied from the Data Sheet, www.digikey.com ) 
For the phototransistor the circuit used was as in fig.4.5:

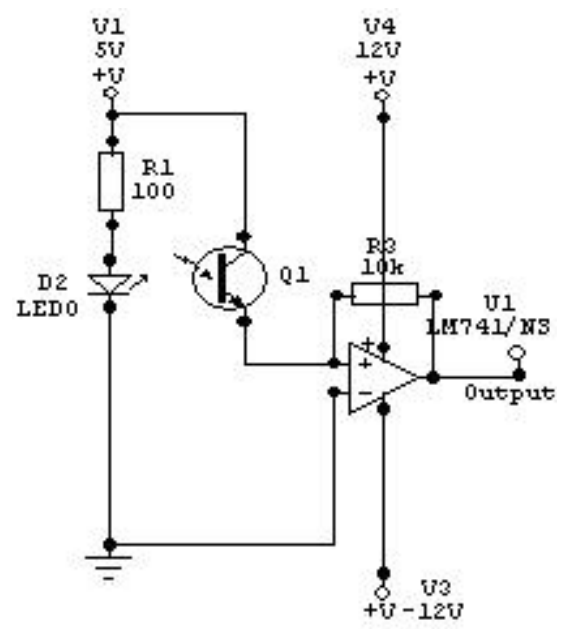

Fig.4.5 Trans-impedance Amplifier for phototransistors

This circuit again is a trans impedance amplifier, with a smaller gain, this time, because the transistor already has Beta (close to 100) as a factor multiplying Iin. Surprisingly, the classic LM 741 will do a perfect job, being internally compensated and having a reduced frequency bandwidth (acting like a filter for the high frequencies produced by noisy environment). As phototransistors, PNA 1401L, a silicon NPN from Panasonic was used, and PNZ 108, also silicon NPN from Panasonic, but having signal mixing capability through a base pin. (See the Data Sheet at www.digikey.com )

\subsection{Conclusions}

The design challenges of optical preamplifiers and small signal amplifiers have been in optimizing the trade-offs between sensitivity, speed and trans-impedance gain.

The trans-impedance configuration was successfully used as an interface-buffer with the optical sensors. From an input of around ten microamperes, the voltage at the output of the trans-impedance amplifier was in the range of tens of volts. As a trans-impedance amplifier, the IC TL071 was used. A voltage amplifier with a gain of 175 was needed to bring the magnitude of the signal in the volts range. The voltage amplifier was implemented through an instrumentation op amp, the INA126 from Burr-Brown. 


\section{Chapter 5 FILTERING UNWANTED FREQUENCIES}

Photo-Sensors and amplifiers are prone to external or internal noise. External noise is due to ambient light and EMI (electro-magnetic interferences). Internal noise is due to the noise introduced by the devices used or by the misuse of the devices.

\subsection{Introduction}

In the given design the sensor operates in a noisy environment. The signal at the output of the Analog Block has to be cleaned from external or internal parasitic frequencies. A perfect cleaning cannot be achieved with simple circuits, but filtering the unwanted frequencies with electronic filters can drastically reduce the effect of most parasitic frequencies. Because in this project an ECG like response is expected (see fig.1.1) at the output of the Analog Block, frequencies higher than $10 \mathrm{~Hz}$ can be rejected with a help of a Low Pass Filter, without interfering with the key frequencies that form the useful part of the signal.

The strategy used to reduce unwanted frequencies has three main branches:

- Ambient light rejection

- $\quad$ EMI rejection

- $\quad$ Reducing internal noise by using low noise devices in the optical preamplifier, voltage amplifier and filter.

Ambient light affects by generating additional shot noise at the photodiode and by superimposing an additional light signal on top of the desired signal. Additional noise shot reduces the sensitivity of the detector but is hard to be removed since it is a white spectrum added to the desired signal. The ambient light signal can be electrically filtered if it is constant or varying at a much higher (lower) frequency than the desired signal. Incandescent and fluorescent lamps are the two most common artificial light sources. Incandescent lamps radiate a good part of their spectrum in the infrared and are supplied with power directly from the line voltage. Their intensity is modulated with the power line frequency $(60 \mathrm{~Hz})$ and its higher harmonics. Fluorescent lamps driven by passive ballasts will add $60 \mathrm{~Hz}$ and higher harmonics, also. Fluorescent lamps driven by electronic ballasts will emit relatively low levels of infrared, but they will produce periodic light fluctuations with harmonic components up to $1 \mathrm{MHz}$. [Narasimhan, 1996]. Those harmonics are too high to interfere with our ECG-like signal.

The EMI is present because of electromagnetic parasitic fields, due mainly to the electromagnetic field of the wires coming to or from the line voltage supply and apparatus containing switching power supplies. The switching frequency harmonics cannot be completely rejected by classic filters; this is the reason for their existence.

The lowest parasitic frequency considered important for this project is $30 \mathrm{~Hz}$. So the decision is to apply a low pass filter having $10 \mathrm{~Hz}$ as the corner frequency, and a flat pass band. 


\subsection{Low Pass filters}

A low pass filter, as shown in figure 5.1, passes low frequency signals, and rejects signals at frequencies above the filter's cutoff frequency. (The cutoff frequency or "corner frequency" is the frequency value from where the magnitude of the signal suffers more than $3 \mathrm{~dB}$ attenuation)

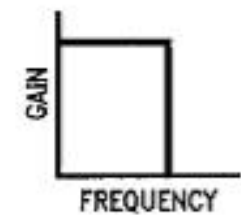

(a)

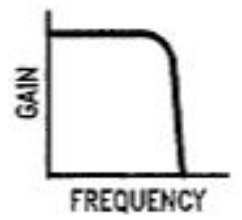

(b)

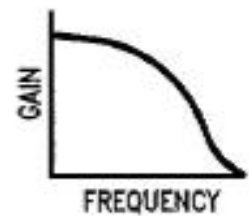

(c)

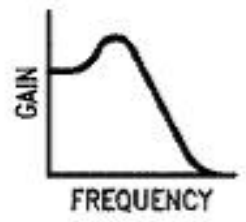

(d)

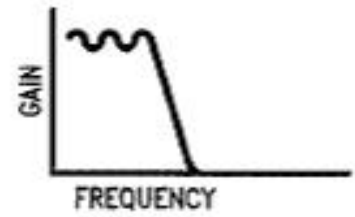

(e)

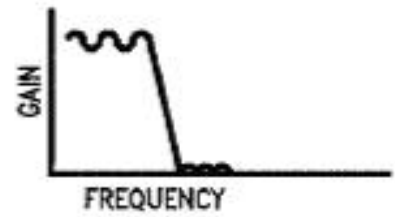

(f)

Examples of Low-Pass Filter Amplitude Response Curves

Fig.5.1 Low-Pass Filter Responses, according with the approximation used: a) Ideal, b) Butterworth, c) Bessel, e) Chebysev, f) Elliptic; (from [24])

Butterworth approximation is used in this project filter implementation. It is called also the "maximally flat response", because it exhibits a nearly flat pass-band response with a roll-off of $20 \mathrm{~dB} /$ decade for every pole. It was considered that Butterworth approximation, due to its response, will interfere less than other approximations with the shape of the amplified photo sensor signal (See fig.5.1.b)).

\subsection{The Main Characteristics of a Second Order Filter}

In electronic design the second order filter is the basic cell. (second order comes from the order of the equation describing the denominator of the transfer function) For simplicity, the general approach to build higher order filters is to cascade $2^{\text {nd }}$ order filters).

To be able to design a filter one needs to know the filter's main characteristics:

1. The Filter Type (low pass, high pass, etc.)

2. The Center Frequency ( $3 \mathrm{~dB}$ attenuation)

3. The Filter $\mathbf{Q}$. (the quality factor of the filter - will determine the relative shape of the amplitude response.

4. The pass-band gain (filters can be built with any gain, sometimes unity gain is considered for simplicity).

Note: Usually, because of the level of calculations, computers are used for filter design problems. 


\subsection{Switched-capacitor filter (SCF)}

(For the principle of operation of the SCF see Appendix A.B1)

Overcomes some of the problems inherent in active filters, adding some new capabilities.

Advantages:

- Don't need external capacitors or inductors (some of them don't even need resistors, such as the one used for this project application, Max291 low pass filter)

- Allows consistent, repeatable design using inexpensive crystal-controlled oscillators.

Disadvantage: are sensitive to temperature changes.

\subsection{Conclusion}

Each type of filter has advantages and disadvantages. For the Low Pass Filter to be used in this project, the Butterworth approximation was chosen due to its flat pass band curve. As a practical approach, the switched capacitor (SCF) is generally much easier to design comparing with active filters and definitely more efficient than the passive filters. In conclusion, a Low Pass Filter, with Butterworth approximation, was implemented through the SCF approach.

\subsection{Choosing the filter for the actual application (The IC Max 291 - Butterworth, Low Pass, $8^{\text {th }}$ order, SCF filter)}

The simplicity of the Max291 solution versus other SCF filters, like LMF100 (National) is obvious. For the Maxim's approach there is no need for external components at all. There is just the external clock, setting the corner frequency, as can be seen in fig.5.2, 5.3. (In contrast, for example, in the National Semiconductor LMF 100 approach, for a $4^{\text {th }}$ order Butterworth, 5 external resistors are needed).

See the MAX291 data sheet and web site at www.maxim-ic.com for ordering info from Dallas Semiconductor (MAXIM).

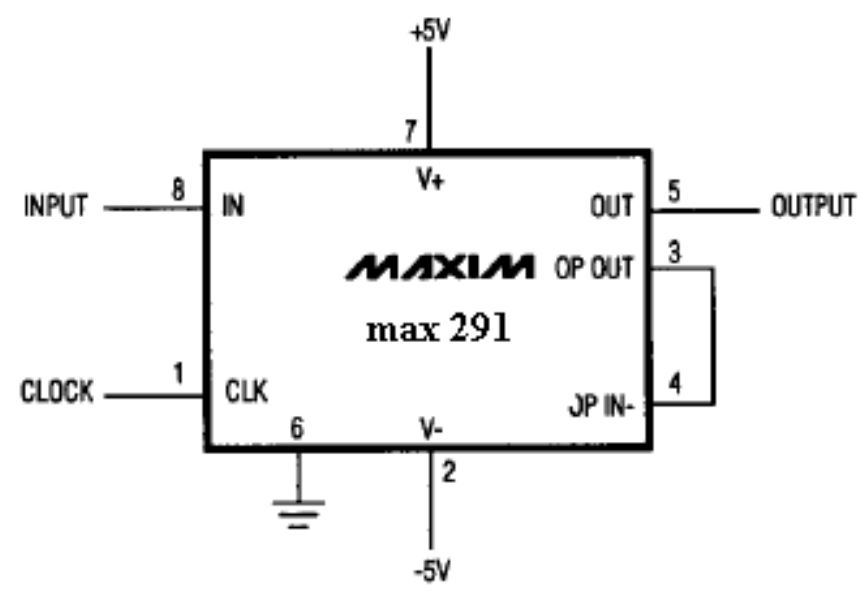

Fig.5.2 Low Pass $8^{\text {th }}$ order SCF, dual power supply filter.

(From the data sheet, www.maxim-ic.com ) 
The clock signal, for simplicity, can be provided either from a classic timer, as an LM 555, or even simpler, from a programmable timer, using a PIC 12F629 (maybe the smallest MCU (micro controller), 8 pin DIP, from Microchip Technology).

As fig.5.3 and 5.4 show, this solution also is based on simplicity and efficiency. With a quartz crystal or a resonator, very accurate oscillators can be built using the specified PIC. The best that can be achieved with a RC oscillator, with a Wien bridge or an LM555, is $0.1 \%$. An LC oscillator will do approximately $0.01 \%$ (ten times better). But with a well-designed crystal oscillator, a few ppm (parts-per-million) accuracy can be reached ([24]). It requires an external resistor for the MCLR pin (master clear / reset), if one needs to stop or start the clock at specific times. Otherwise, the $4^{\text {th }}$ pin of the PIC can be wired to VDD $(+5 \mathrm{~V})$. The program code in C (for the PIC12F629 - as $1 \mathrm{KHz}$ oscillator) very simple and intuitive, can be seen in Appendix A.C1.

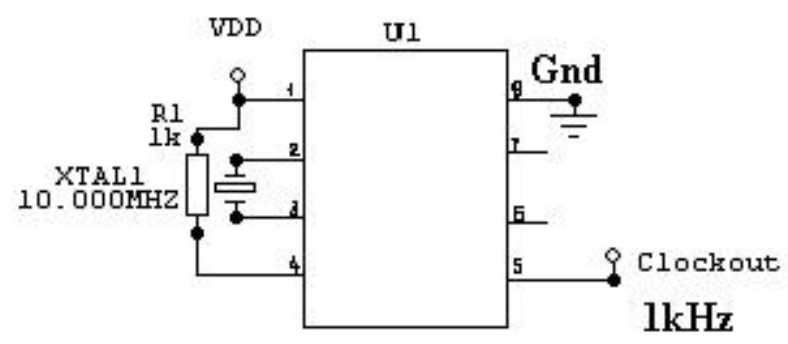

PIC $12 \mathrm{~F} 629$

Fig.5.3 The 1KHz clock generator for the SCF build with Max 291

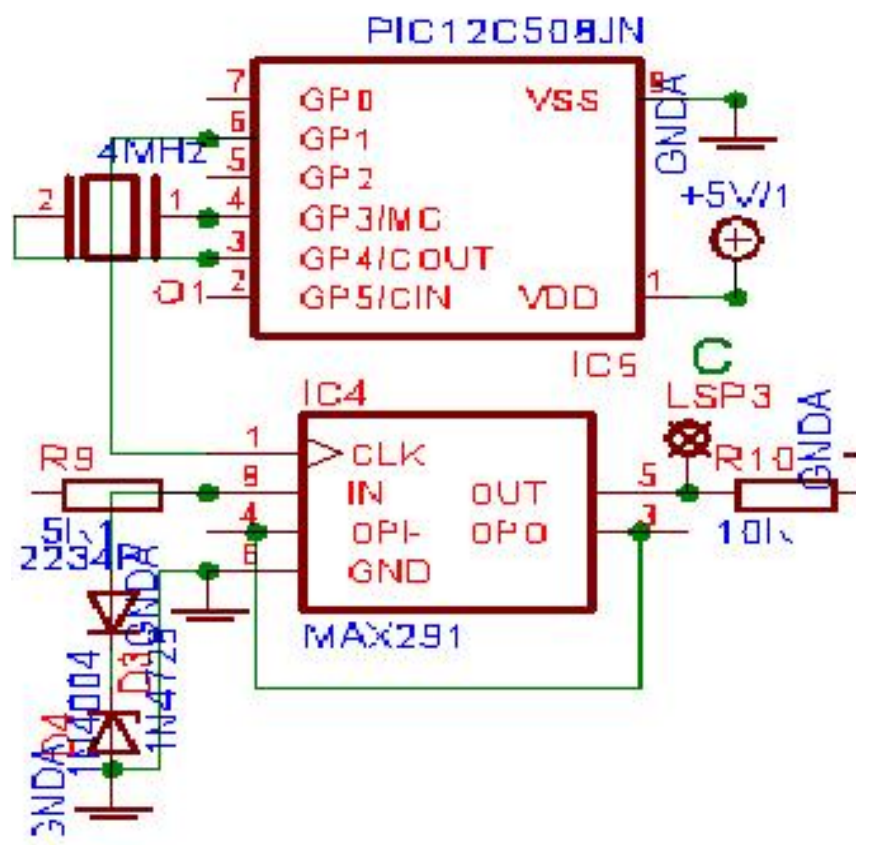

The Low Pass Filter implementation

Fig.5.4 The Low Pass Filter together with its external components

The fig.5.4 shows a detail from the whole electronic schematic containing the SCF and its external components: the PIC MCU as the $1 \mathrm{KHz}$ oscillator and a $4 \mathrm{MHz}$ crystal. 


\section{Chapter 6 SIGNAL PROCESSING}

\subsection{ECG-like signal, blood pressure}

The signal coming out of the reflective optical sensor after being amplified and filtered looks like an ECG signal. To understand better how to process an ECG-like signal, some anatomical features of the heart have to be considered.

The acronym "ECG" stands for electrocardiogram, which represents the electrical activity of the heart muscle cells and it is the signal obtained at the skin surface of the human body.

At the surface of the skin, ECG is created when depolarization (activation) and the following re-polarization spread in the whole heart muscle.(for more details see [32])

The signal registered at the skin surface originates from many simultaneously propagating activation fronts, at different locations, which affects the size of the total component.

ECG

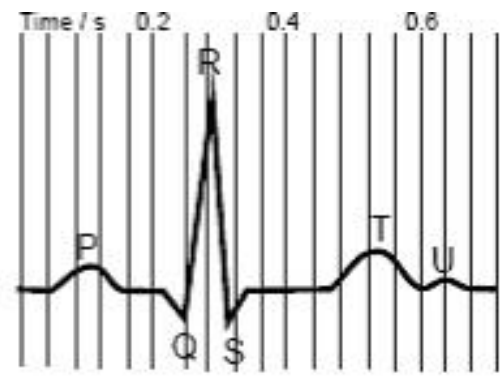

Fig.6.1 Standard ECG

(adapted from [32, page2]). P-wave is generated by atria, QRS is Ventricle depolarization and $\mathrm{T}$-wave is ventricle repolarization)

\subsubsection{Heart Mechanism}

The activation of the heart has to be synchronized somehow. The sino-atrial node is formed by specialized muscular fibers located in the upper part of the right atrium, in the base of the superior vena cava. The sino atrial node produces spontaneously the heart frequency (approximately $60 \mathrm{bpm}$ (beats-per-minute)), which is independent of the nervous activity. When necessary, the rate can be varied and controlled by the sympathic and parasympathic nervous system. The path of the activation process propagates from the sino atrial node to the right and to the left atrium muscle tissue. From atria to ventricles the path of activation propagates through the atrioventricular node. The task of this node is to delay the start of the ventricles activation, permitting enough time for the blood to fill the ventricles (due to the atrial contraction) before the ventricles will contract. The atrioventricular node has its independent activation frequency (approximately $50 \mathrm{bpm}$ ) in case the stimulation from the sino-atrial node doesn't come at the right time. The depolarization continues then through the ventricles. The rear-upper parts of both ventricles and septum depolarize last. The stage of re-polarization starts from the outer cells and proceeds as a front towards the inner wall. (See [32] for more details) 


\subsubsection{ECG propagation along the whole body}

The whole human body forms a volume conductor, the bioelectrical phenomena occurring inside the "conductor" generate a signal measurable at the skin surface.

While the depolarization front propagates towards a positive (by definition) electrode, a positive voltage can be seen in the measuring device. Similarly, the potential difference developed by the depolarization front propagating in the opposite direction is negative. Because the potential difference over the membrane caused by the repolarization is in the opposite direction as in the depolarization, also the voltages caused by "re-polarization front" show as reversed compared to the propagation direction.

\subsubsection{Blood pressure}

Fig.6.2 shows how the blood pressure changes during different stages of action of the heart.

- The highest (systolic) pressure - is reached during the contraction of the ventricles.

- The lowest (diastolic) pressure - is reached during the rest stage.

- The pulse pressure - is the difference between systolic and diastolic pressures.

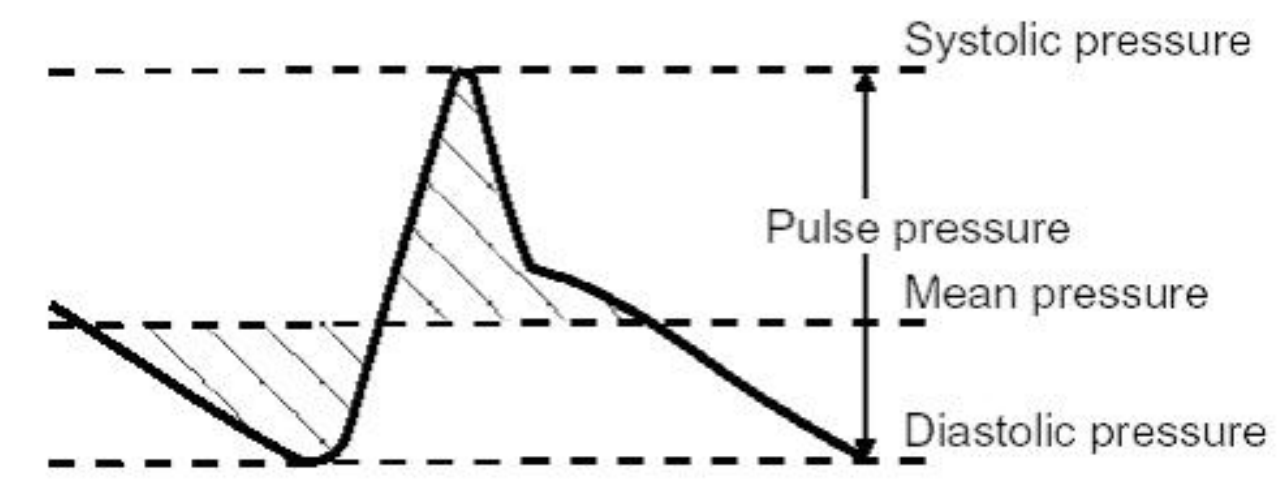

Fig.6.2 Blood Pressure Wave-shape (from [32], page5).

The expression mean pressure - is used to describe the average of the arterial pressure, which is:

Mean pressure $=($ diastolic $\mathbf{p} .+$ systolic p. $) / 3([32]$, page 5$)$ 


\subsubsection{Comparison}

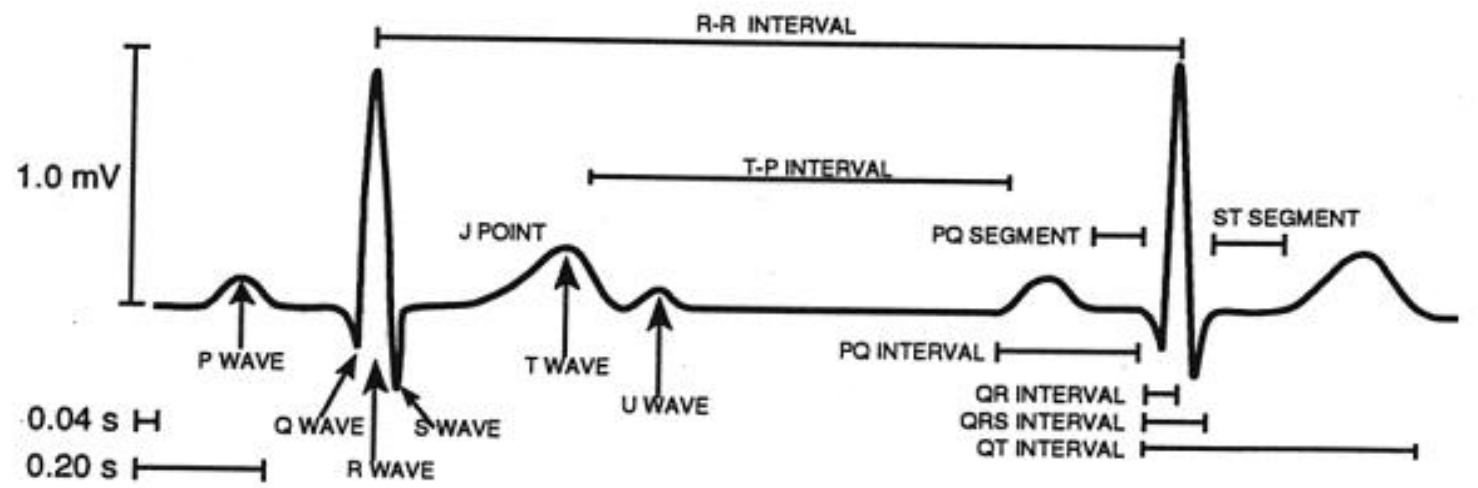

Fig.6.3 Standard ECG Signal, main features used in physiology (from [32], http://www.ee.tut.fi/rgi/kurssit/71221/ekg analyysi/ECGANALY.html)

A comparison is made between the shape of a standard ECG signal (obtained after an electric measurement) and our Photo-Plethysmographic signal (obtained after the photo-sensors has converted the variations of the blood amount in a capillary region, in an electric signal)

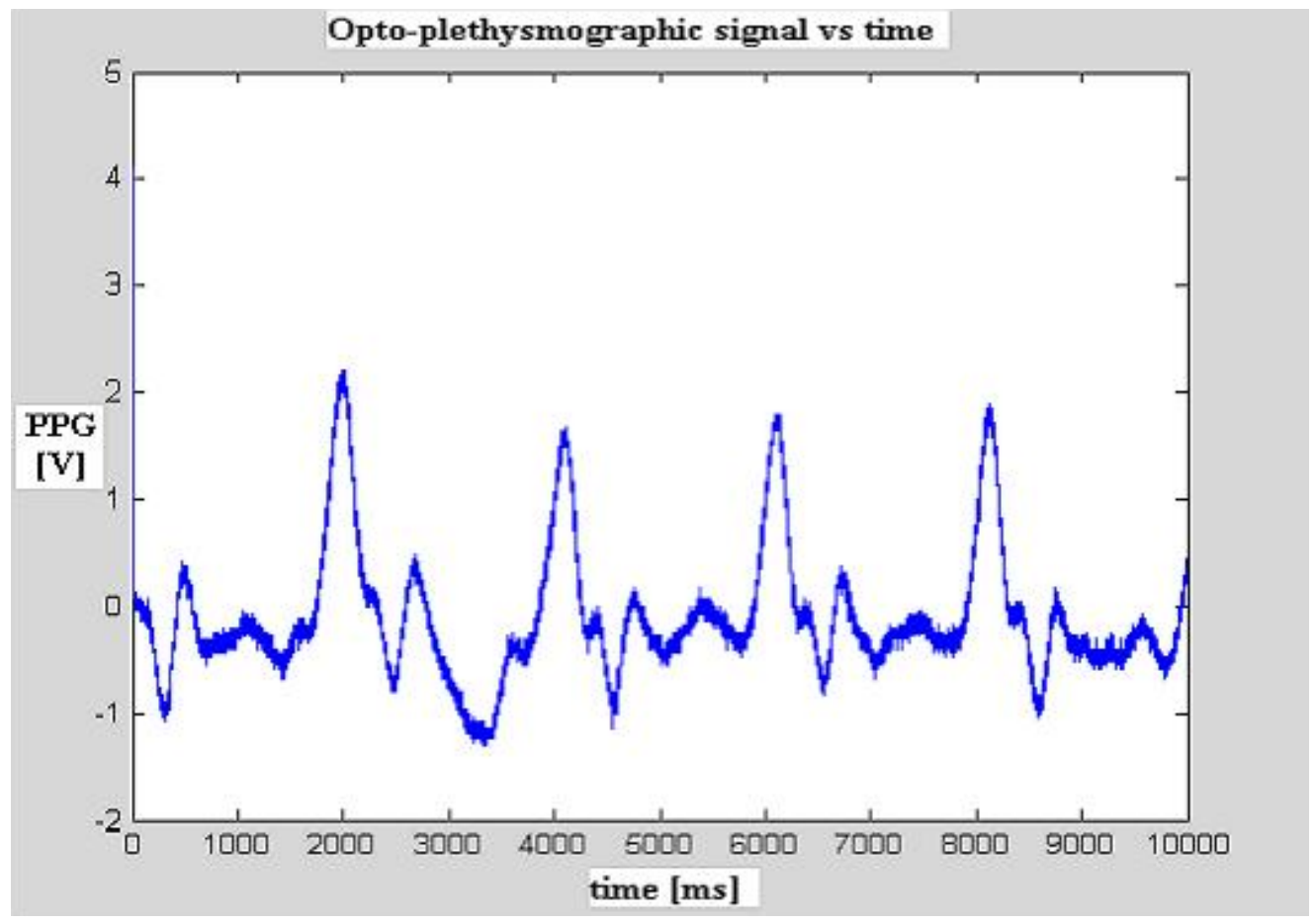

Fig.6.4 Photo-Plethysmographic wave-shape, obtained through photo-sensors

As one can see, these two signals have almost the same shape. They have almost the same main characteristics (emphasized in the upper picture.) 


\subsection{Conclusions}

1. The photo-plethysmographic signal obtained at the level of the skin, from a finger or from the face of the palm with the optical sensor system, amplified and filtered, has a lot in common with an ECG signal. (See P, T and QRS complex on both signals, they look very much alike.)

2. Because ECG witnesses the heart mechanism at work, the photo-plethysmographic signal can be considered a good enough method to test human liveness. (e.g. from a dead hand or a spoof hand no periodic ECG-like signals will be detected, no matter what materials the spoof is made of.)

3. To process this ECG-like signal, classical processing methods used in ECG analysis can be applied.

\subsection{Processing the ECG signal to decide upon liveness}

\subsubsection{QRS complex recognition}

The accurate detection of the R-peak of the QRS complex is the prerequisite for the reliable function of ECG-analyzers. The recognition of almost all ECG parameters is based on a fixed point identifiable at each cycle. The R-peak is suitable for use as the datum point, because it has the largest amplitude and sharpest waveform that can be extracted from the ECG. The time and amplitude measurements can be performed when the apex of the R-peak is detected at each cycle.

There are a large number of recognition algorithms used in ECG-analyzers and in many cases the principles of operation vary. Some are based on different types of amplitude triggering, while the others examine the signal in the frequency domain. The adaptive properties of algorithms to the changing signal may differ and some algorithms use statistical methods for identification.

Note: The interpretation of ECG signals is a typical application of pattern recognition. An experienced cardiologist can easily diagnose various heart diseases just by looking at the ECG printout. In some specific cases, sophisticated ECG analyzers achieve a higher degree of accuracy than that of cardiologists, but at present there remains a group of ECG changes that are too difficult to identify using computers.

\subsubsection{Brief example of a typical method of parameters determination}

(example from: Biomedical Eng. Lab., Ragnar Granite Institute -Tampere University of Technology - Finland, [32])

There are on-line measurement systems (real-time), and off-line systems (when the analysis is performed afterwards.

- In the on-line measurements systems the parameters are calculated separately for each cycle.

- For the off-line systems a representative sample is formed out of the measured cycles and is used in analysis. The more typical methods to form representative samples are average and median cycle construction. 
Averaging procedure: after the R-peaks are detected, the average of the voltage value of the R-peaks at each cycle is calculated. The same procedure is repeated for $\mathrm{R}+1, \mathrm{R}+2$, etc., samples at each cycle and so on, until the end of the cycles. This procedure is also applied in the reversed direction (R-1, R-2, etc.).

Median Calculation: In principle the median calculation is performed similarly, using the same time period of cycles and taking the median value of the data points at each cycle. Measured parameters in a standard method.

The basic parameters obtained from an ECG signal are depicted in the next figure.

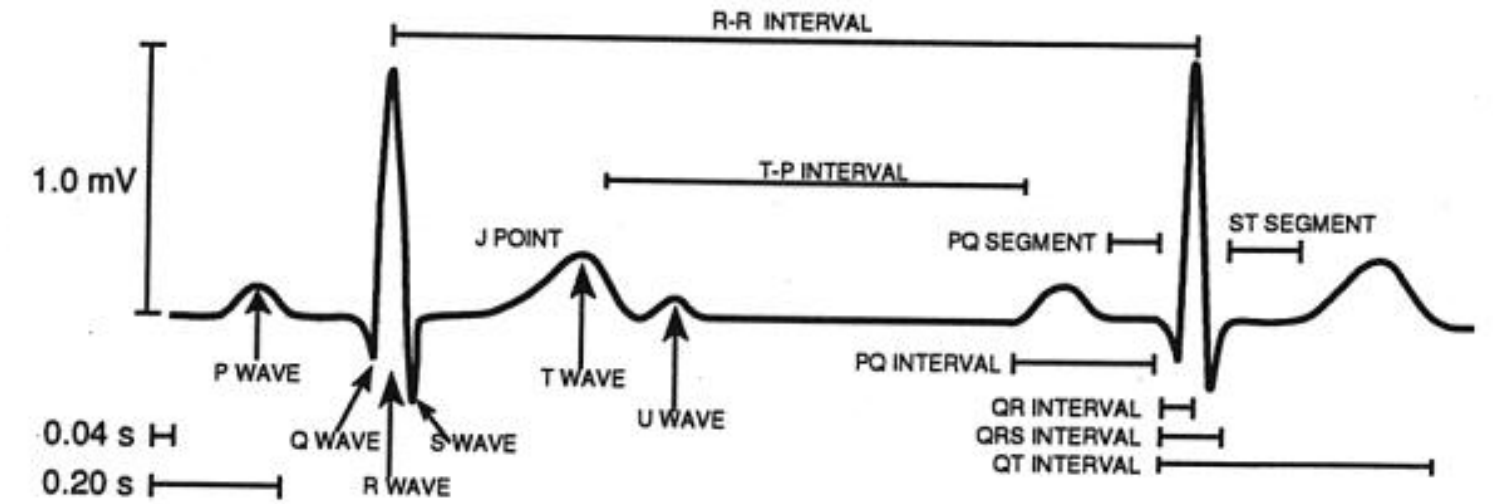

Fig.6.5 Standard ECG signal (from [32])

The parameters obtained from a measured signal are dependent upon the application. In addition to R-peak, the datum point, it is essential to determine the baseline of a signal, which is defined as the line between two consecutive P-Q intervals. All amplitude measurements are compared to the baseline.

Steps to follow for the software determination. (example taken from [32])

1. Read the signal.

2. Design a low pass filter $(15 \mathrm{~Hz}$ or $30 \mathrm{~Hz})$. ( $2^{\text {nd }}$ order IIR or FIR- Filter-Lab).

3. Apply the filter to the signal.

4. Differentiate the signal with 2-point, or 3-point or 5-point methods. The result is to be used in R-peak detection.

5. Square the differentiated signal.

6. $\quad$ Detect the R-peaks

7. Correct the baseline of the original signal

8. Generate median or average segments from the original signal. 


\subsection{Important ideas for our project (R-peak detection)}

The main goal of studying different methods of analysis of most important parameters is to get the simplest and most efficient way to detect liveness from an ECG-like signal. After research a decision was made that the main and only parameter that should be detected in order to decide upon liveness is R-peak detection.

R-peak has two main features that can help in its' detection:

1. It has the biggest magnitude from the ECG spectrum.

2. It causes the highest frequency component of the ECG spectrum.

In hardware, if the highest magnitude parameter of a complex signal needs to be detected, usually a threshold will be applied, with the help of a voltage comparator. The disadvantage is the variation in magnitude of the signals obtained from different persons, so this will complicate the circuitry by adding an AGC (automatic gain control) stage, in order to maintain the magnitude at the input of the comparator in the same range. (or the threshold can be adaptive, but this is not as intuitive as the previous method).

In software, the so called "amplitude level triggering method is based on the square of the derivative. Knowing that R-peak values contain the highest frequencies of the ECG spectrum, differentiating the filtered ECG signal will give the high frequency components. The reliability of the identification of R-peaks can be increased by squaring this differentiated value, (will highlight the high frequency components). The R-peaks will be detected by determining the known amplitude level as a triggering level. The triggering level can also be adaptively determined, thus the value of the triggering level is dependent on the upper values of the ECG signal. Usually a PC computer (or a high level DSP) is necessary to perform the steps described earlier).

Note: One of the best examples of adaptive triggering through software is the IEEE paper "A Software Trigger for intra-cardiac waveform detection with Automatic Threshold Adjustment", by J. Jenkins and others, from Univ. of Michigan (See [9]).

One can think to use analog computers. Analog Computers still find application in engineering practice in the simulation of dynamic systems.

The Analog Computer is a device that is based on three operational amplifiers circuits: the amplifier, the summer and the integrator. These three building blocks, permit the construction of circuits that can be used to solve differential equations and simulate dynamic systems.[28].But, beside the fact that the analog computers are obsolete in the information era, the price of high complexity of the circuitry will be paid.

\subsection{Conclusion}

For liveness to be detected, R-peaks through the thresholding method described earlier (hardware) should be identified. The disadvantage of different magnitudes for different people should be taken in consideration (see Further Work, Analog Block, in Chapter 9, Paragraph 9.2 of this thesis), using an AGC stage with a PGA (programmable gain amplifier). Details of the circuitry used in R-peak detection and implementation are discussed in Chapter 7, The Logic Block. 


\section{Chapter 7 LOGIC BLOCK}

\subsection{Block Diagram}

This block represents an interface between the Sensors and Analog Block and the Decision Block, which has to give an accurate resolution concerning the hand liveness.

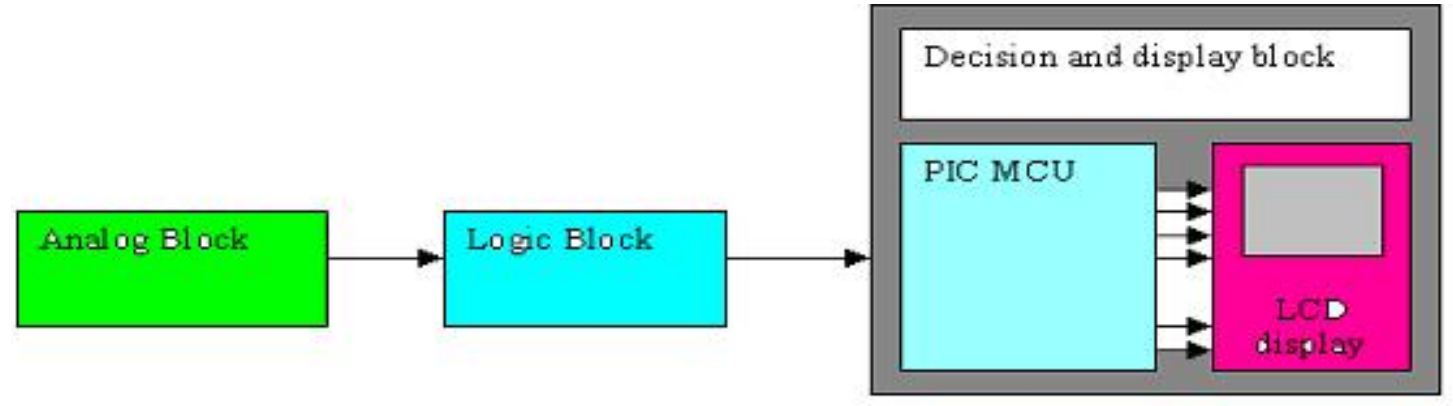

Fig.7.1 Block Diagram of the whole Project

An explanation was previously given for the analog block and its components. Further, an explanation for the functionality of the logic block in detail is given.

The Logic Block is an interface between the Analog and Decision Blocks.

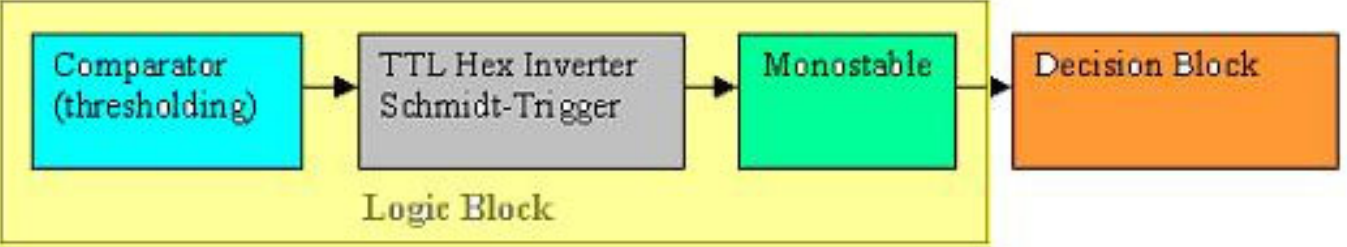

Fig.7.2 The Logic Block Diagram

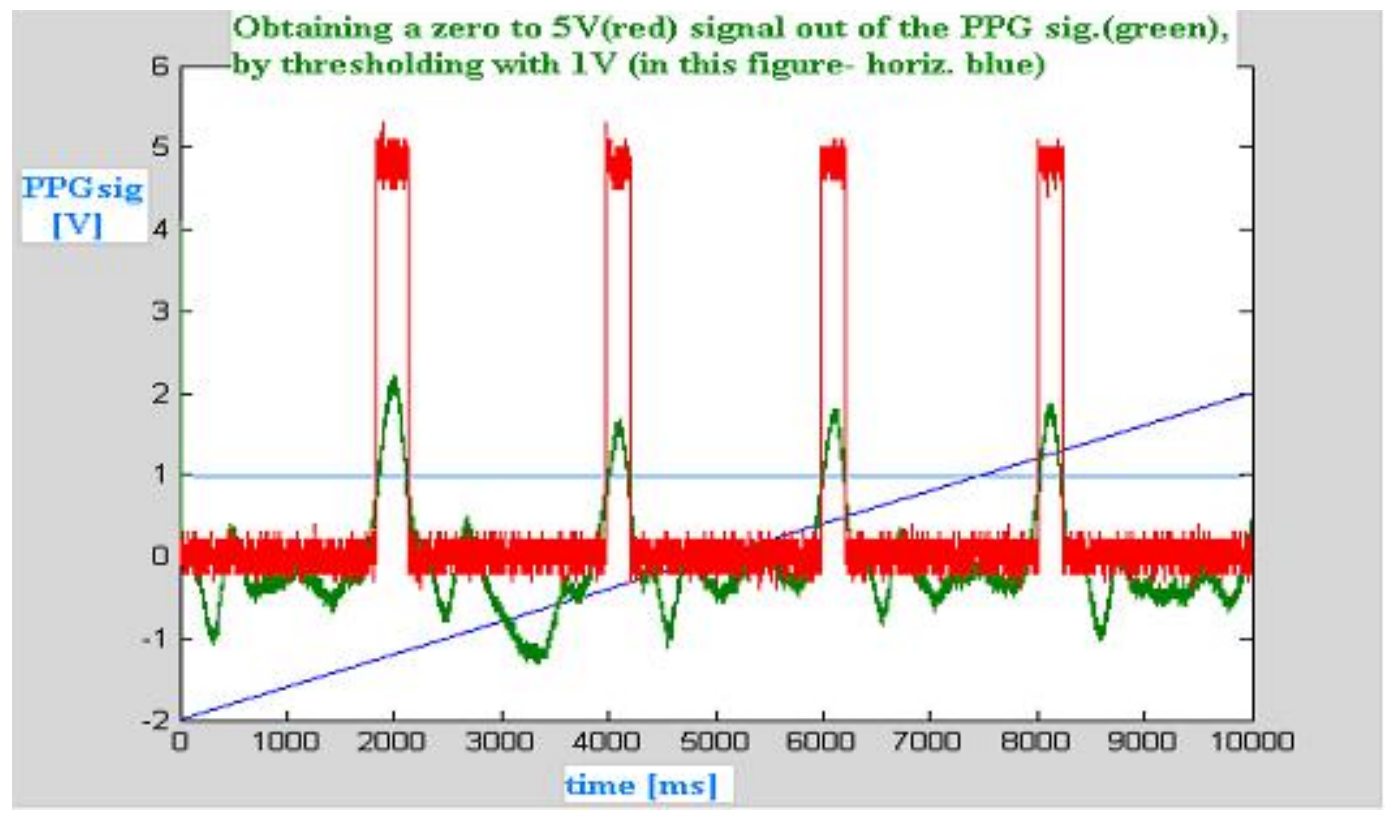

Fig.7.3 The input \& output signals of the comparator LM 311, for a threshold $=1 \mathrm{~V}$ 


\subsection{The presentation of the details of the schematic of the logic block}

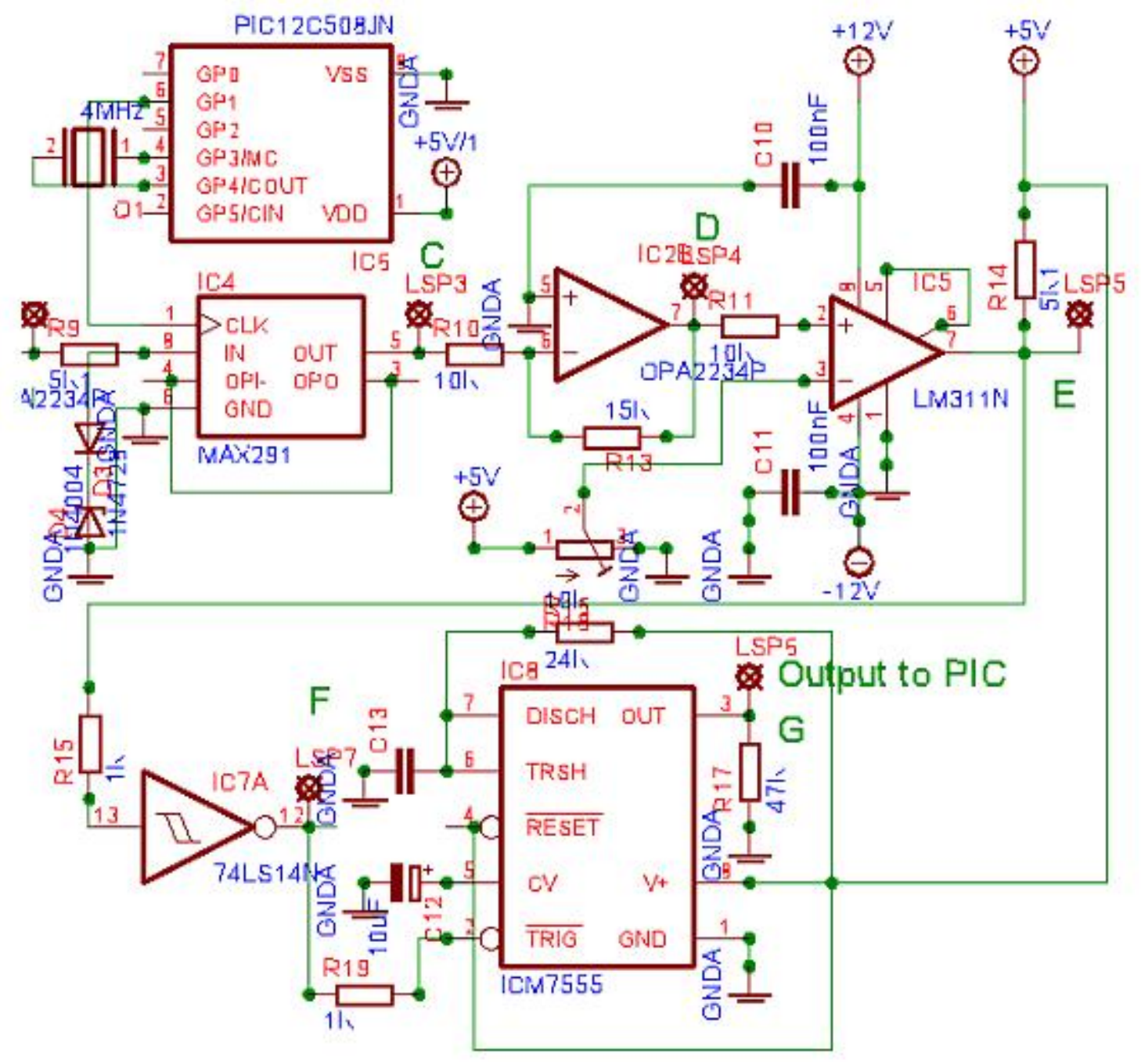

Fig.7.4 Detailed electronic schematic of the Logic Block and the last stages of the Analog Block

The fig.7.4 shows the interaction of the logic and analog blocks. IC2B is the second half of the dual op amp OPA 2334. This is the last amplifier stage and chapter 10Improvements and further work, will show that instead of $\mathrm{R} 8=25 \mathrm{~K}$ adjustable, the AGC loop will be implemented. The AGC loop is necessary to prevent big differences in signal magnitude at the input of the voltage comparator (LM311), which has a fixed threshold. (The idea of an adaptive threshold may be considered here, instead of AGC loop).

The decision block is interfaced with the logic block because the PIC MCU accepts TTL or CMOS signals, but the analog (like-ECG) signal goes up to $6-7$ Volts, and down to 
$-2,-3$ Volts. The interest is in the periodicity of the signal not in its shape (for this application of detecting liveness). A measurement of the period of the signal, measuring the R-R intervals will be made. Based on this and some simple computations done inside the MCU, the LCD will display:

1. The pulse rate in [beats per minute].

2. A warning in the case that artifacts come out.

3. A message for the situation in which the system accepts the subject as being alive: "Live detected!"

4. Another message, on the same lines of the LCD, prompting the user to try again. The role of the voltage comparator is to convert the analog (ECG-like) shape of the signal into a rectangular pulse of the same period.

\subsubsection{LM 311 brief presentation}

(for an exhaustive documentation, see the data sheet at www.national.com )

LM311 is a special-purpose IC package available as voltage comparator.

Advantages for this application:

- accepts relatively large inputs.

- has provisions for selecting the desired reference voltage levels (sometimes those are internally clamped to a specified voltage range)

- offers the strobe option on pin 6 (with a TTL compatible signal)

- provides an open-collector output (as shown in the figure), which allows the user to connect the output of the open-collector transistor to any supply voltage of choice, by means of an external pull up resistor. (the open collector transistor operates in saturation mode, thus making an easy task for the user to calculate it, not being critical (as it would be in an amplifying application)).

For $5 \mathrm{~V}$, usually one can choose between hundreds of ohms and couple thousand ohms. Ex.: 1 to $5 \mathrm{~K}$ can be a good choice, and the output of the comparator will switch between 0 and $5 \mathrm{~V}$, according to the decision resulting after the input comparison.

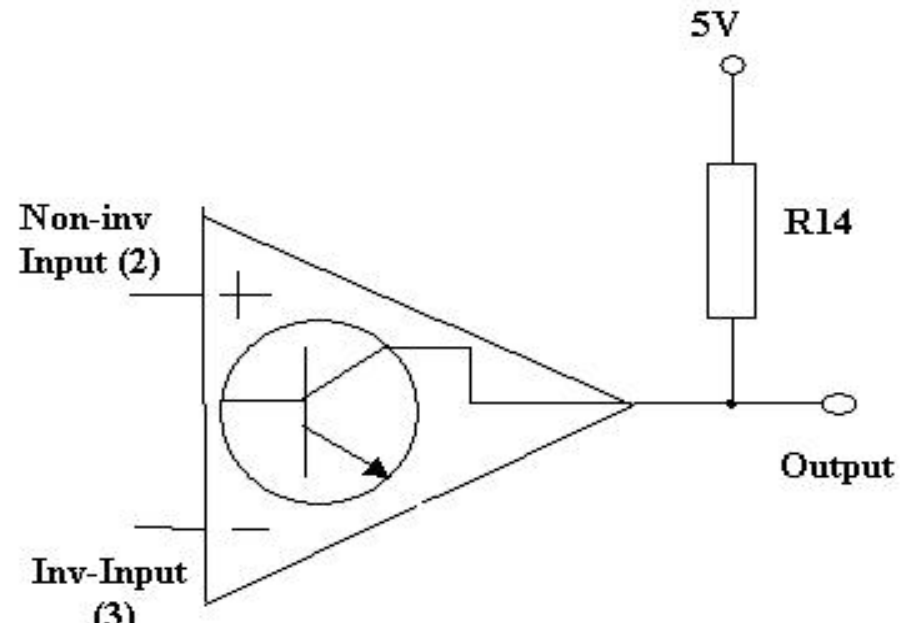

Fig.7.5 LM 311, detail with the Open Collector output 
The PIC inputs cannot be connected directly to the signal coming out of the comparator, first because some pulses would be so thin (narrow) that the PIC will ignore them, and second because more "noise cleaning" can be done with the help of a Schmidt Trigger.

\subsubsection{SN74LS14 (Hex Inverter, Schmidt Trigger)}

The role of the Hex inverter Schmidt Trigger is double. First it has to invert the signal, to make it suitable for the 555-monostable input.
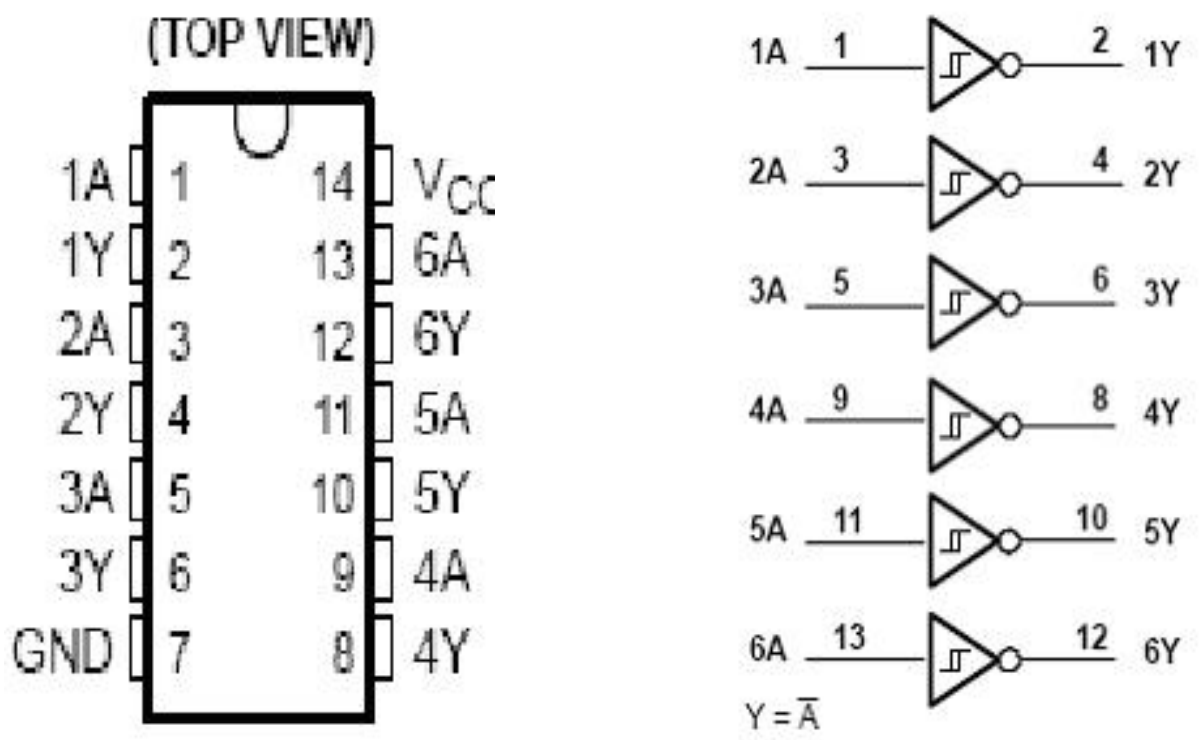

Fig.7.6 Internal organization of the Hex inverter SN 74LS14

The Trigger Schmidt, Hex Not Gate (fig.7.6), is a TTL gate of type 74LS14. This type (LS - low power Schottky technology) was chosen because of it is very low power consumption. (For this application there is no need for a bigger Fan-out capability). Basically, the 555 IC in a monostable configuration will be sensitive on the High to Low input transitions on the pin2 (trigger input). So first the 74LS14 IC will invert our signal and then, due to the Schmidt Trigger property, it will introduce a hysteresis helping us to get rid of more noise, that could cause false triggering of the monostable. 


\subsubsection{LM 555 (Monostable configuration)}

(See the data sheet on www.digikey.com )

Finally, the monostable is enhancing to an optimal width, the rectangular pulse becoming readable by the PIC input. (See fig.7.7 with waveforms taken from Data Sheet)
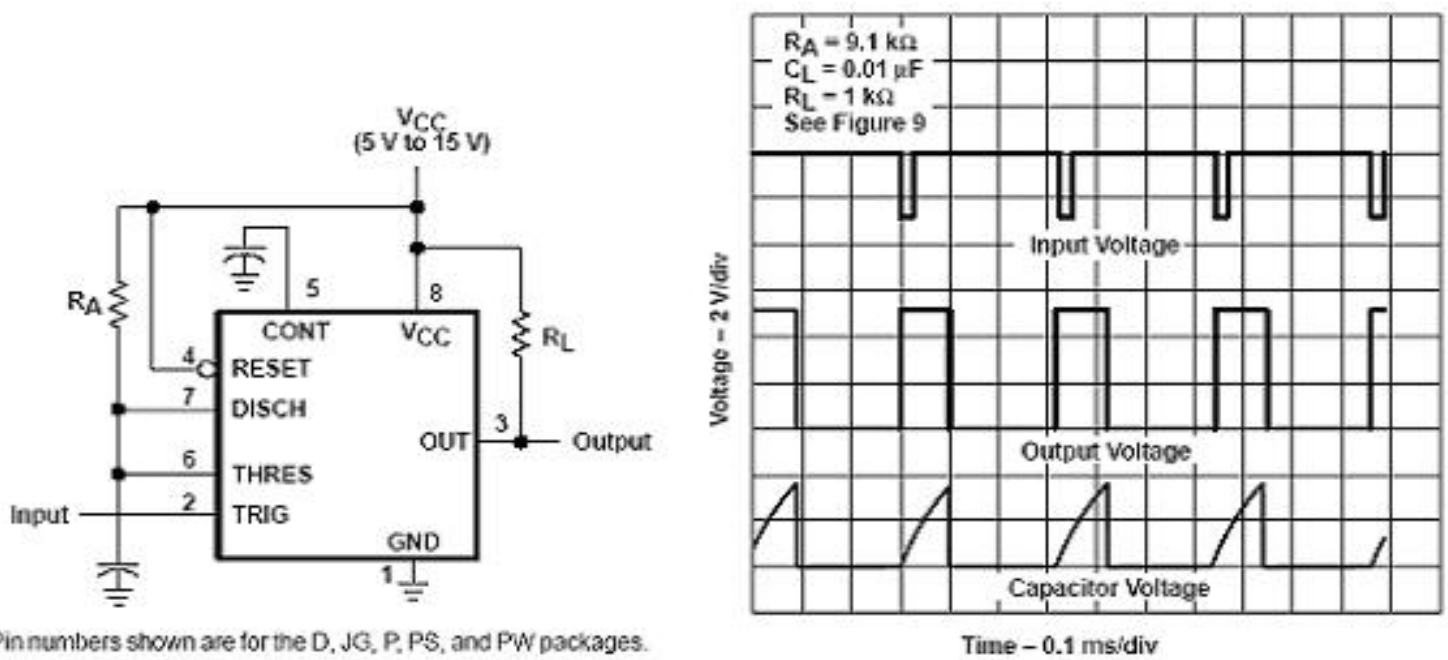

Pinnumbers shown are for the D, JG, P. PS, and PW packages

Fig.7.7 Left: LM555 - Monostable Standard configuration Right: Input (2), Output (3) and Discharge (7) Capacitor Waveforms (from data sheet)

In the left side of the fig.7.7 the standard monostable configuration is shown.

LM555 (NE555) is a timer circuit, capable of producing accurate time delays (pulses) or oscillation. An RC network controls the time delays. IC 555 is a multipurpose IC, able to perform, both, monostable and astable (clock) functions.

The main advantage of this configuration (as opposed to the same configuration built with BJTs, MOSFETs or OP Amps, lies in the greater accuracy and repeatability, the ease of designing an application and the flexibility provided by the 8 pin package, integrated configuration.

The width of the signal coming out of the monostable with 555 is given by the following formula ([28]):

$\mathrm{T}=1.1 * \mathrm{R} 18 * \mathrm{C} 13$

where: $\mathrm{R} 18=24 \mathrm{~K}, \mathrm{C} 13=10 \mathrm{uF}$;

$\mathrm{T}=$ width of the signal in [s];

The calculation started by imposing the value of T, to be $25 \mathrm{~ms}$.

Second step: Pick up the value of $\mathrm{C} 13=10 \mathrm{uF}$. After this the formula is applied to find the $\mathrm{R} 18$ value $=24 \mathrm{~K}$; for verification: $\mathrm{T}=1.1 * 24000^{*} 10^{*} \mathrm{e}^{\wedge}(-6)=25 * \mathrm{e}^{\wedge}-2[\mathrm{sec}]=25 \mathrm{~ms}$. 


\subsection{Analog and Logic, Schematic and PCB (practical considerations)}

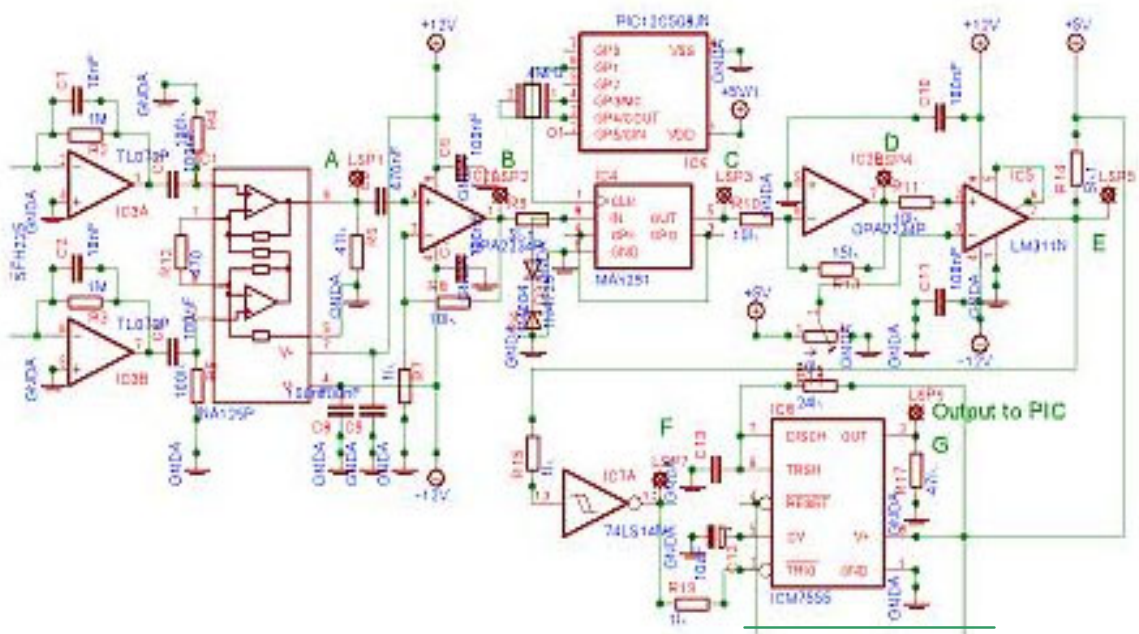

Fig.7.8 Detailed Schematic, Analog plus Logic Blocks

To build the schematic, containing new Ics and specialized Op Amps, three advanced software programs were tried:

1. Circuit Maker (Trax Maker) Version 6.2.c Pro (1999) - full version

2. Eagle 4.11 (non-profit version)

3. Protel DXP (one month trial version).

- Circuit Maker does not permit building libraries (at least the version mentioned). It allows the creation of "Macros", sort of customer designed items. But this is very time consuming.

- Protel DXP is a very expensive software (around $\$ 9,000$ for the full version), comparable in price and achievements with Orcad. (Circuit Maker is a subdivision of Protel and Eagle a subdivision of Orcad (Cad-Soft))

- Eagle 4.11 was the most friendly, in terms of adding libraries and browsing fancy Ics. I could find almost anything, if not directly, I could go on line and download lots of libraries available.

Note: One disadvantage of Eagle, compared to the other two mentioned, is that once settled for a value of an item in the schematic, one is not able to move just the value of the item, but the whole item plus the value and name. This is the reason that the schematic has poor legibility for some devices.

The PCB implementation through Eagle 4.1 software is presented in Appendix A.B3. 


\section{Advices for practical implementation of devices and components to the PCB}

- For soldering a temperature controlled soldering station is highly recommended. The traces will be very thin and can be easily exfoliated if overheated.

- Care must be taken of the ground loops, especially when low signal amplifiers are involved. Those can create noisy currents and ringing (parasitic oscillations). Plan the grounding scheme carefully. If the circuit has a lot of digital circuitry, separate grounds and power planes have to be considered.

- Decouple the amplifier power supplies with bypass capacitors, as close to the amplifier as possible. For J-FET and C-MOS amplifiers, a 100nF capacitor is recommended. Also, decouple the power supply with a $\mathrm{C}=10 \mathrm{uF}$ capacitor.

- Use short leads lengths to the inputs of the amplifier.

- Always read carefully the specifications from data sheet, prior to design and use a new device. (e.g. you have to know what input range is required for your amplifier. If both inputs will go beyond the specified input range, the output will typically be driven to one of the power supply rails.(for more details, see [13] and [25]).

Note1: It is very difficult to remove digital switching noise from an analog signal. So care has to be taken in designing and implementing the circuit power supplies.

Note2: The amplifiers built on proto-boards or "bread-boards" will not work perfectly. Noise and oscillations may occur due to long wires (acting like antennas) and imperfect connections. 


\section{Chapter 8 DECISION BLOCK}

This block's resolution is most important for achieving the goal of our project. Is the hand presented on the platen fake (impostor) or human (genuine)?

\subsection{Block Diagram}

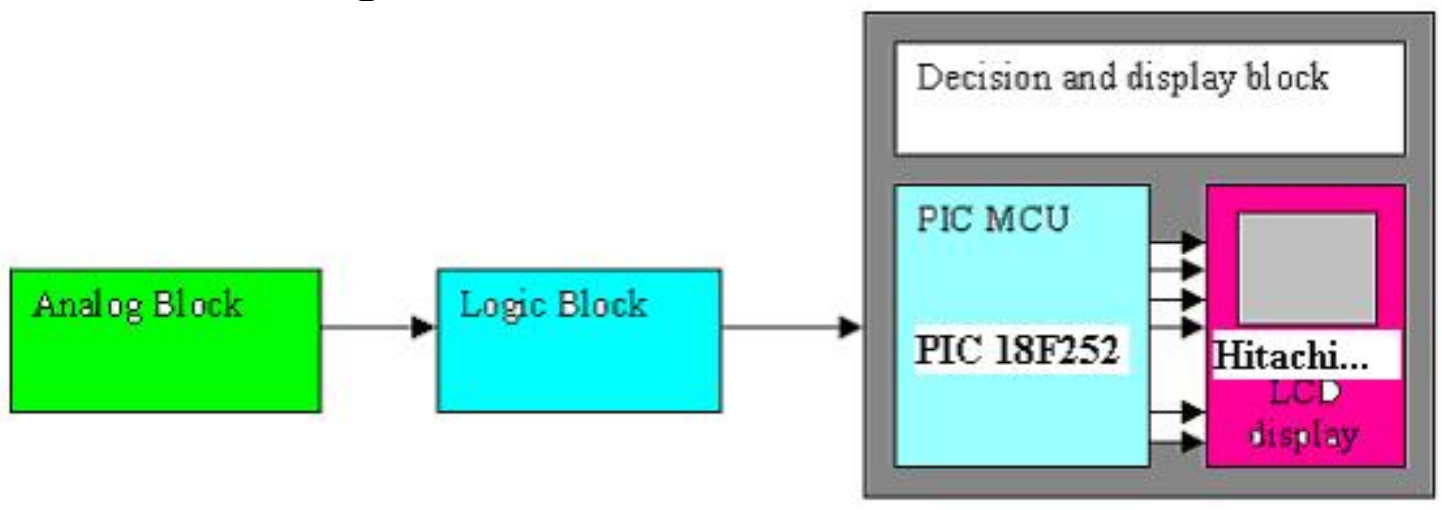

Fig.8.1 The whole design block diagram

The logic block creates the interface for the decision block, PIC 18F252 and LCD 20434. The "brain" of the decision block is the PIC Micro-controller, which reads the rectangular pulses coming into $\mathrm{C} 2$ (pin 17) of the PIC, counts them and registers the values counted. The count value is processed and compared in software with preloaded values, and checked to be in a certain range: greater than a preset "P1" value (corresponding in the real world to $50 \mathrm{bpm}(\mathrm{bpm}=$ beats per minute) $)$, and smaller than a preset "P2" value (corresponding to $150 \mathrm{bpm}$ ). The reason we chose those values for P1 and P2 lies in the assumption that a healthy individual has a heart rate in the range 60 to $120 \mathrm{bpm}$ ([20]). Our range is larger because the resolution in the given method is sacrificed for the sake of simplicity.

1. In principle, if the above condition (count $>\mathrm{P} 1 \&$ count $<\mathrm{P} 2$ ) is met, the decision of Liveness detected will be displayed on the LCD screen, line 3.

2. On the contrary, when the condition is not fulfilled, the message will be, instead, try again or please stand still!

The second outcome occurs when the PIC MCU has at the input either no pulses at all (spoof probable), or there are too many artifacts and the period of rectangular pulses arriving at the PIC input is highly affected by movement artifacts.

Note: The Hand Geometry Scanner will reject enrolled people that are not able to keep their hand still, while verifying!

3. Although not required, but nice feature to have, a pulse rate in [beats per minute] is displayed on the line 1 of the LCD. 


\subsection{Presentation of the PIC MCU}

\subsubsection{Microcomputer and Micro-controller}

a) A microcomputer is a computer in which the CPU (central processing unit) is a microprocessor. The purpose of a microcomputer is general.

The best example is the PC (personal computer) - a general-purpose computer.

b) In contrast, when a controller (or computer) is embedded into some device, for some other purpose than general purpose computing, it is said to be an "embedded controller", or micro-controller. The micro-controller is also called: a "one chip solution". A micro-controller, typically includes:

- CPU.

- RAM

- EEPROM.

- I/O (input / output) Ports - to communicate with CPU (or from CPU to exterior) either parallel or serial (RS232 or SPI).

- Timers and counters.

- Interrupt controller.

Applications of micro-controllers

1. Consumer Segment - Home Appliances, Entertainment equipment.

2. Automotive - keyless entry, ABS (anti-lock braking system), airbags.

3. Office Automation - PC keyboards, copiers, printers.

4. Telecommunications - Cell phones, pagers, photo cameras, fax-machines, answering machines.

5. Industrial Automation - Door locks, automatic faucets, industrial machines

6. Biomedicine - health monitors, etc.

\subsubsection{The PIC MCUs}

The PIC series of MCUs (micro-controllers) are a product of Microchip Technology Company.

Among Microchip products (like analog circuits and memories) are the PIC (peripheral interface controller) micro-controllers production, and their development tools.

PIC single chip processors are low cost $(<\$ 7)$, have many integrated peripherals such as: A/DC, PWM, CCP, CAN, Digital I/O, I2C, RS232, SPI, PSP, many interrupt possibilities and FLASH, RAM and EEPROM memory. Easy to learn instruction set with just 35 instructions in Assembly and a host of C-Compilers for high level programming. Microchip has a very generous sampling program offering 5 samples totally free, each 90 days.

Microchip attracted a big percentage of the 8-bit micro-controller market customers, because they invested a lot (money and intelligence) in the development tools for their PIC devices, eventually succeeding to bring them to the customers, at low prices. The common user can take advantage of the development tools, for a complete solution: 
- Complete environment - MPLAB

- Embedded Workbench for PIC micro-controllers (IAR)

- $\quad$ Language tool for code development (assemblers, C-compilers)

- Simulators

- Emulators

- Debuggers.

- Programmers

MPLAB environment, supports all kinds of PIC s (Microchip produces around 50 types (!) and development tools.

Compared to their main competitors (8-bit MCU s), the Basic Stamps (BS1, BS2, from Parallax company) the PIC s have 2 major advantages:

- PIC is 20 to 100 times faster than a Basic Stamp, because the Program Memory can be accessed parallel. (the stamps are storing the program memory in a serial EEPROM Memory, serial data access being much slower).

\subsubsection{PIC's basic arhitecture and other features}

The basic architecture of PIC $\mathrm{s}$ is the Harvard architecture, with a RISC set of instructions. This is in contrast with the von Neumann architecture, that is used extensively in general purpose microprocessors manufacturing, which shares the Program Memory and Data Memory (See the $\mu \mathrm{P}$ 68HC11, Intel 80486, Pentiums, etc.).

In the Harvard architecture, the Program Memory and Data Memory are not shared and are even on separate busses. This allows the program instructions to be fetched and executed concurrently with the data, resulting in higher reliability and speed.

In a CISC (complex instruction set computer) architecture, some of the instructions take as many as 5 clock cycles to execute, while in a RISC architecture (as in PIC) usually it takes one clock for one instruction (only very few instruction need 2 cycles (clocks)).

All PIC family are "single chip operation" (SCO) and part of them come at a very inexpensive price (the PIC xxCxxx family - OTP s, one time programmable), but with the same set of instructions.

\subsubsection{Some Disadvantages of PICs}

1. The PIC has limited Memory. This fact greatly limits the complexity of conventional assembly programs that can be implemented. Therefore, simplified solutions, with simple mathematical models should be implemented.

2. Besides the PIC18XXX, the rest of the family has a non-linear RAM memory that is difficult to access. The register file map of the RAM is organized in four banks (waste of instructions to change from one bank to another). 


\subsubsection{Brief overview of PIC $18 F 252$}

(For details see Appendix A.17, or www.microchip.com )

a) PIC 18F 252 is a 28 pins (DIL 28) high performance, Enhanced Flash micro-controller, with 10-bit AD.

b) Memory Organization:

- Linear Program Memory addressing to 32Kbytes (Flash).

- Linear Data Memory addressing to $1.5 \mathrm{Kbytes}$ (RAM).

c) Capability of 10 MIPS operation.

d) 4 timer/counter modules. Two capture / compare / PWM (CCP) modules.

e) Master synchronous Serial Port (MSSP) module, with 2 modes of operation:

- 3-wire SPI (supporting all 4 SPI modes)

- I2C Master and Slave mode.

f) Addressable USART module (supports RS-232 and RS-485).

g) Parallel Slave Port (PSP) module

\subsection{LCD (Liquid Crystals Display) - Optrex DMC20434}

\subsubsection{General characteristics}

LCDs are able to add a lot to an application, in terms of providing a useful interface for the user (with a professional look) but also while debugging an application. During the last few years the prices of LCDs have decreased, so now one can find a lot of nice bargains to buy an alphanumerical LCD.

The LCD used for this project contains the most common type of LCD controller, a sort of standard for industrial applications: HITACHI 44780.

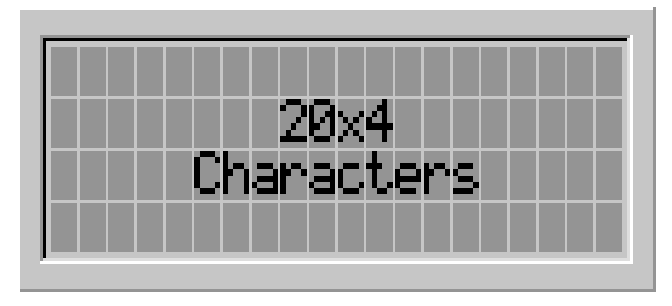

It provides a relatively simple interface between a processor and an $\mathrm{LCD}$, for a low cost. The most common connector used for the 44780-based LCD is the 14 pins in a row (PSIL parallel / series in line). The following table contains Optrex DMC20434 LCD pin-out: 


\begin{tabular}{|c|c|c|c|c|}
\hline \multicolumn{5}{|c|}{ Pin assignment for $<=80$ character displays } \\
\hline $\begin{array}{c}\text { Pin } \\
\text { number }\end{array}$ & Symbol & Level & $1 / 0$ & Function \\
\hline 1 & Vss & - & - & Power supply (GND) \\
\hline 2 & Vcc & - & - & Power supply (+5V) \\
\hline 3 & Vee & - & - & Contrast adjust \\
\hline 4 & RS & 0/1 & I & $\begin{array}{l}0=\text { Instruction input } \\
1=\text { Data input }\end{array}$ \\
\hline 5 & $\mathrm{R} / \mathrm{W}$ & $0 / 1$ & 1 & $\begin{array}{l}0=\text { Write to } L C D \text { module } \\
1=\text { Read from LCD module }\end{array}$ \\
\hline 6 & $E$ & $\begin{array}{c}1,1-- \\
>0\end{array}$ & 1 & Enable signal \\
\hline 7 & DB0 & $0 / 1$ & $1 / 0$ & Data bus line 0 (LSB) \\
\hline 8 & DB1 & $0 / 1$ & $1 / 0$ & Data bus line 1 \\
\hline 9 & DB2 & $0 / 1$ & $1 / 0$ & Data bus line 2 \\
\hline 10 & DB3 & $0 / 1$ & $1 / 0$ & Data bus line 3 \\
\hline 11 & DB4 & $0 / 1$ & $1 / 0$ & Data bus line 4 \\
\hline 12 & DB5 & $0 / 1$ & $1 / 0$ & Data bus line 5 \\
\hline 13 & DB6 & $0 / 1$ & $1 / 0$ & Data bus line 6 \\
\hline 14 & DB7 & $0 / 1$ & $1 / 0$ & Data bus line 7 (MSB) \\
\hline
\end{tabular}

The LCD's interface is a parallel bus, allowing simple and fast reading/writing of the data to and from the LCD.

\subsubsection{Operation modes}

When an alphanumerical LCD is written, the data waveform coming into the input will write an ASCII byte out on the LCD's screen. The ASCII code to be displayed is 8 bits long and is sent to the LCD, either 4 or 8 bits at a time.

The four-bit mode: Two nibbles of data (sent high 4 bits, and then low 4 bits, with an "E" clock pulse with each nibble) are sent to make up a full 8-bit transfer. " $\mathrm{E}$ " is the enable clock pulse, used to initiate the data transfer within the LCD. Choosing between the 2 modes of operation is a decisive option for an LCD application.

Eight-bit mode: is best used when the application is required to be fast, and $10 \mathrm{I} / \mathrm{O}$ pins are available.

Four-bit mode: require just six bits. To wire an MCU to an LCD in four-bit mode just the top 4 bits of data (DB 4 to 7) are written to.

Note: The four-bit mode was chosen for the present design, for simplicity.

For details on the instructions available for use and other information on the Optrex DMC 20434, the reader can check [15], pages 271- 278 (or the data sheet at www.optrex.com). 


\subsubsection{Initialization of the LCD. Building an LCD driver.}

Before one can send commands or data to the LCD module, it has to be initialized. In 4bit mode one will write to the LCD in terms of nibbles. (A nibble is one half of a byte, the upper four bits, or the lower four bits).

The transmission of a byte goes first sending the High Nibble, then the Low Nibble, and the enable clock pulse "E" pin is toggled each time that four bits are sent to the LCD. This project uses a 4 lines times 20 characters LCD, as pictured on the previous page. A "C" code subroutine "lcd" (the LCD driver) was written and included as a preprocessor directive header: \# include <lcd.h>. It was mapped on the Port A (pins 2 to 6, or A0 to A5) of the PIC 18F252. (Please see Appendix A.C4 for the LCD driver code "lcd.h" and the entire $\mathrm{C}$ code (there are 2 versions for the Decision Software, with the same LCD driver, mapped on the same port.))

\subsubsection{Connecting the Optrex DMC 20434 LCD with the PIC}

\begin{tabular}{|c|c|c|}
\hline PIC 18F252 (pins) & Optrex (pins) & Comments \\
\hline Pin \# 5 (RA3) & Pin\# 14 & \multirow{4}{*}{ Data Bits 4, 5, 6, 7 . } \\
\hline Pin \# 4 (RA2) & Pin\# 13 & \\
\hline Pin \# 3 (RA1) & Pin\# 12 & \\
\hline Pin \# 2 (RA0) & Pin\# 11 & \\
\hline \multirow[t]{2}{*}{ Pin \# 6 (RA4) } & Pin\# 6 & "E" - enable clock pulse \\
\hline & Pins\# 10, 9, 8,7 & Not used \\
\hline GND (means: write) & Pin\# 5 & $\mathrm{R} / \mathrm{W}-\mathrm{read} /$ write bit (no reading from) \\
\hline Pin \# 7 (RA5) & Pin\# 4 & $\mathrm{R} / \mathrm{S}$ - select data or instructions transfer \\
\hline GND (max contrast) & Pin\# 3 & Contrast Voltage \\
\hline+5 Vcc (470 resistor) & Pin\# 2 & Vcc - Positive DC Voltage supply pin \\
\hline GND & Pin\# 1 & GND - ground pin \\
\hline
\end{tabular}

Note: Pin\# 6 of the LCD ("E" clock), is connected to PIC's Pin\# 6 (RA4) and also pulled up to $+5 \mathrm{~V}$ through a $1 \mathrm{~K}$ resistor.

- Software considerations concerning the PIC

In the "main" part of the code, when the use of printing to the LCD is required, first the function "lcd.init()" is called, and then, through the function "gotoxy()", the place of the cursor control is specified. (e.g.: "gotoxy $(2,5)$ " - meaning that the "printf" C code command, will begin with the cursor positioned in the second line, fifth character.

- Setting the contrast voltage to the display

To set an appropriate value of the contrast voltage on the display, usually a potentiometer ( 10 or 25 ohms) wired as a voltage divider is used. Because in the GND position, the cursor has maximum contrast, the design is simplified by grounding the third pin of the LCD.

Note: Paying the price of slowing down the application, the simplest way of wiring up an $L C D$ (minimum number of wires required) is to use but 2(two) I/O pins of the PIC MCU. This can be built with the help of a shift register, used as a serial to parallel conversion device, first. (For details, see page 278, [15]). 


\subsection{Decision Block Implementation}

The decision block is implemented through 2 versions:

Version 1 - Roll over variant, in which the block is measuring continuously the time interval between incoming pulses, applies an internal algorithm and displays the output ("Live detected!" or "Try Again!") together with the pulse rate on the LCD's screen.

Version 2 - In this version a 6 second sequence is generated, in which the number of pulses received, is counted. According to their number, another algorithm is applied and the resolution ("Live detected!" or "Try Again!") is displayed.

\subsubsection{Version 1 (Roll over measurement)}

Version 1 uses just the PIC 18F252 MCU together with the Hitachi 44780 LCD display. Everything is done through the software embedded in the PIC.

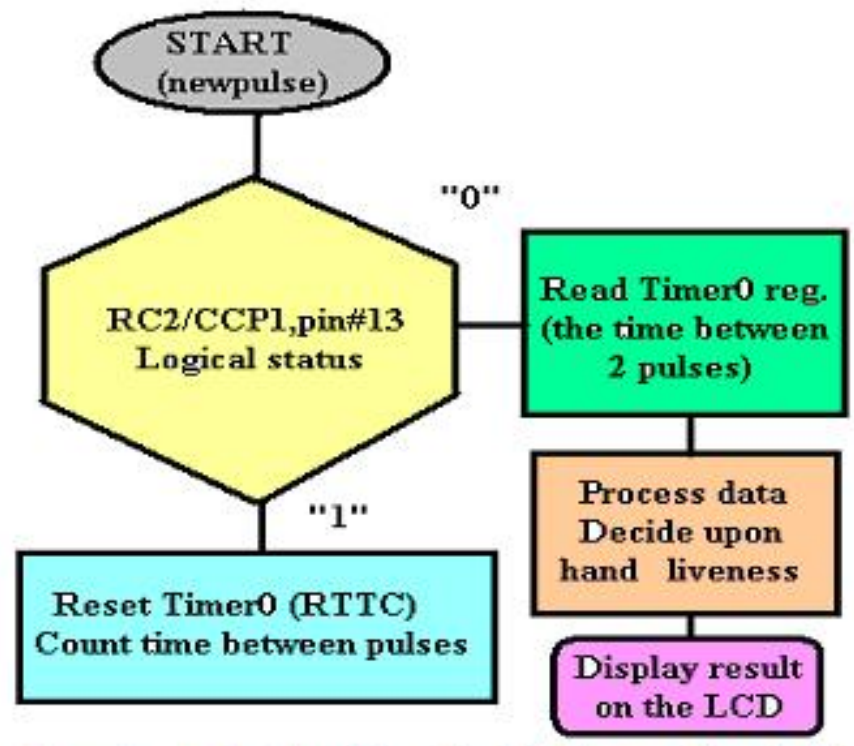

Flow-chart for Decision Block Software, Versionl

Fig.8.2 Flow Chart for Decision Block Software

This is a short variant of the $\mathrm{C}$ code for the Decision Block Soft, version1 (Roll Over): The program starts with the header, preprocessor directives and variable declarations. A Boolean variable "new pulse" is used to simulate the arrival of a new pulse. When a pulse inputs RC2/CCP1 (pin\# 13 PIC18F252) the controller jumps in the capture isr function, (the interrupt subroutine). It resets Timer0 and start counting the time between 2 pulses. When a new pulse arrives at pin RC2 of the PIC the result of the capture is processed, the algorithm decides upon liveness and display the message on the LCD.

See Appendix A.C2 for the C code. 


\subsubsection{Version 2 (The 6 seconds sequence)}

The easiest way to understand the electronic schematic of Decisional block (See figure 8.3 ) is to go through the functional diagram (figure 8.4). Identify the colors.

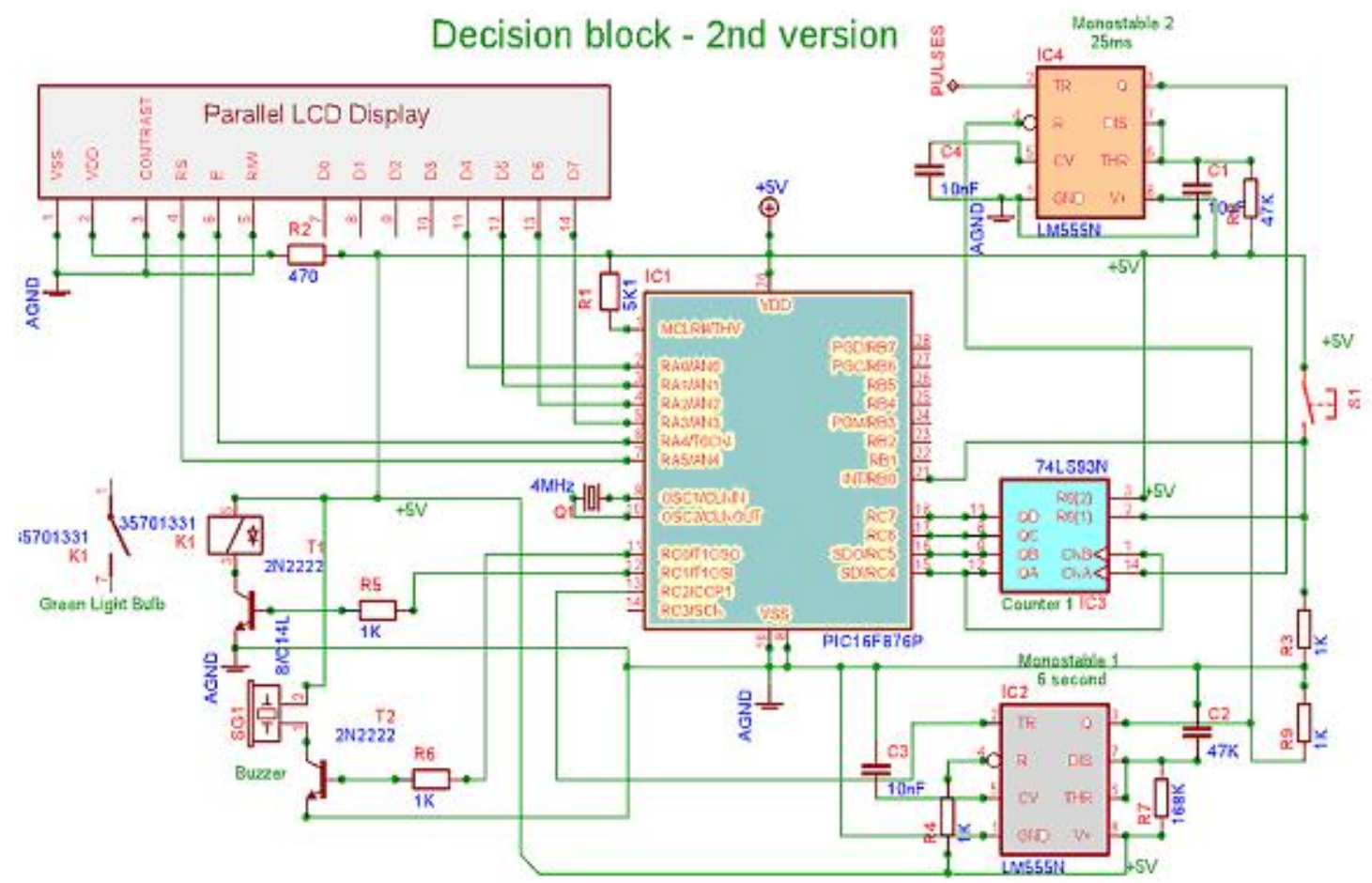

Fig.8.3 Detailed Electronic Schematic for the Decision Block

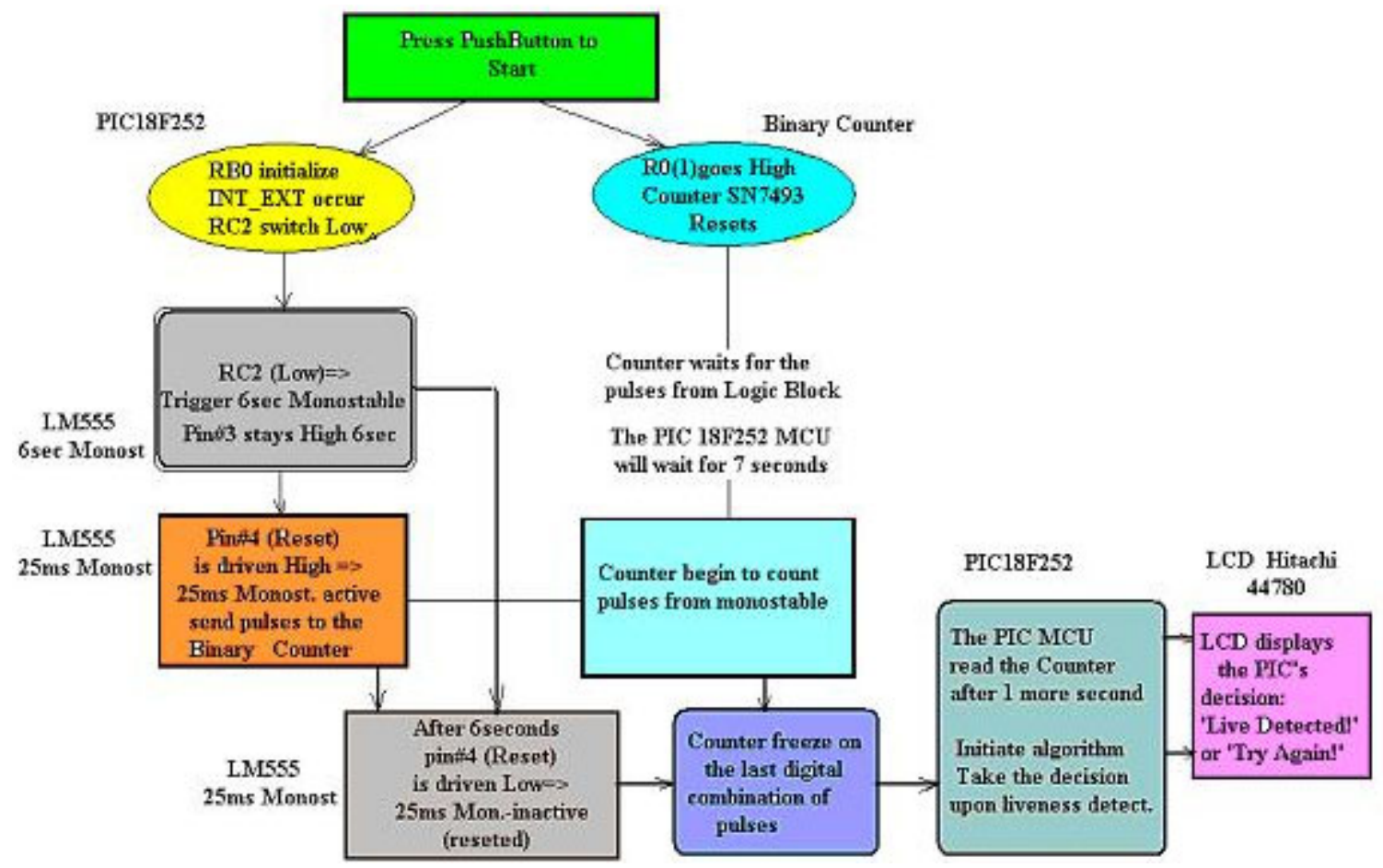

Fig.8.4 Flow Chart with the explanation of Decision Block Schematic 


\section{Explanation of schematic and functional diagram (fig.8.3 and fig.8.4):}

Nothing happens until the pushbutton S1 (red in the right side of the schematic) is pressed. The LCD will be displaying the last measurement and its message.

Pressing S1 is the equivalent of an enable, digital signal, coming from HK2 DSP, when the DSP is ready to build the geometrical template.

Note1: This is a moment when no movements or artifacts coming from the hand or fingers are assumed. This is a constraint already required by the HK2, before adding the live detection feature.

Note2: One goal of this project was to add no more constraints to the HK2 design. But it was assumed the existing ones could be used. (This way, the problem of movement artifacts was practically eliminated.)

a) Once S1 is depressed, two things will occur:

- Input RB0 / INT0 (pin\#21 on PIC18F252) switches from Low to High, that initiates the external interrupt in the PIC MCU.

- R (01), or reset \#1 (pin\#2 on SN7493 Counter) switches from Low to High, resetting to zero all outputs of the binary counter.

b) The External interrupt in the PIC initiate:

- Output RC2/CCP1 (pin\#13 of PIC) switches from High to Low for 50ms, triggering

Monostable 1 (with LM555). The pulse width of this monostable is six seconds. The calculus is simple:

$$
\begin{aligned}
& \mathrm{T}_{\mathrm{h}}=1.1 * \mathrm{R}_{7} \mathrm{C}_{2}[\mathrm{~s}]=1.1 * 120 * 10^{3} * 0.047 * 10^{-3}=1.1 * 5.64=6.2 \mathrm{~s} \\
& \mathrm{~T}_{\mathrm{h}}=6.2 \mathrm{~s}
\end{aligned}
$$

Where: $T_{h}$ is the width of the signal while being High

$$
\begin{aligned}
& \mathrm{R}_{7}=120 \mathrm{~K} \\
& \mathrm{C}_{2}=47 \mathrm{uF} \\
& {[\mathrm{s}]=\text { seconds }}
\end{aligned}
$$

- The PIC MCU starts a NOP (no operation, or waiting) for 7 seconds (one extra second, to be sure the counter will be frozen at the time of reading.)

Note: In this calculation it was assumed that the hand stays on the platen 6 seconds, for a better resolution of the algorithm, but the real time that usually the hand lays on the platen is three to four seconds (as previously specified). $T_{h}$ may be set to a desired value by putting instead of $R_{7}$, an adjustable $250 K$ resistor.

c) Events happening inside the 6 seconds sequence:

- The Reset pin (pin\#4, active Low, of Monostable 2, LM555) is switched High. That make Monostable 2 active, beginning to send $25 \mathrm{~ms}$ pulses to the counter, according to the number of pulses it is been triggered (coming from the comparator, in the logic block).

- The PIC MCU is in a wait position as specified earlier, turning off the LCD's screen.

- The counter counts the incoming pulses, displaying their number in a digital form at its 4outputs $\left(\mathrm{Q}_{\mathrm{A}}, \mathrm{Q}_{\mathrm{B}}, \mathrm{Q}_{\mathrm{C}}, \mathrm{Q}_{\mathrm{D}}\right)$.

d) After the 6 seconds sequence comes to an end:

- The output (pin\#3 of LM555) of Monostable 1 is switched from High to Low.

- The reset terminal (pin\#4 of LM555) of Monostable 2 is switched from High to Low, thus resetting Monostable 2. This will become insensitive to the incoming pulses. It will stop sending pulses to the Counter (SN7493). 
- The Binary Counter will freeze its output digital combination. The outputs combination is ready to be read by the PIC MCU. (the outputs aren't changing anymore).

- The PIC MCU is still in a wait position, driving off the LCD.

e) After 7 seconds:

- The PIC wakes up, reads the digital combination provided by the Counter at its inputs and initiates the algorithm provided by the software code to process and decide upon liveness.

- The LCD will display the pulse rate in bpm (beats per minute), and the decision upon liveness. ("Live Detected!" when successful or "Try Again!" - for fail).

f) Between Sequences:

- The LCD keeps displaying the pulse rate and decision until the S1push-button is depressed again, re-initiating the cycle already described.

\subsubsection{PSpice simulation for the Monostables 1 and 2}

Unfortunately only the student version of PSpice software was available. Thus, it was not possible to simulate the whole circuit. The simulation of some circuits of the Logic and Decision Blocks was considered interesting.

A) Monostable 1 (6 second generator)

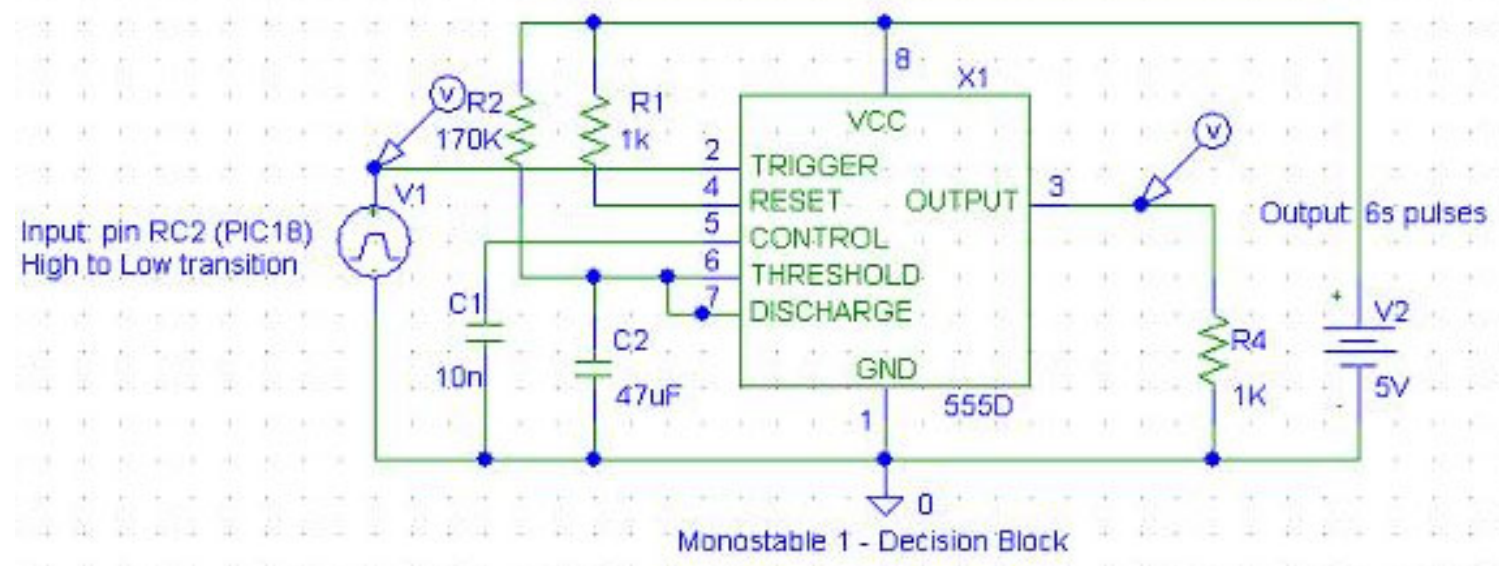

Fig.8.5 PSpice schematic of Monostable 1 from the Decision block

The value of R2 was adjusted from the theoretical calculated. The next two figures, fig.8.6 and fig.8.7 show Voutput (pin 3 of LM555, cyan) versus V1 (pin 2 - Trigger Input, magenta), and just Voutput (green), in the second figure. 


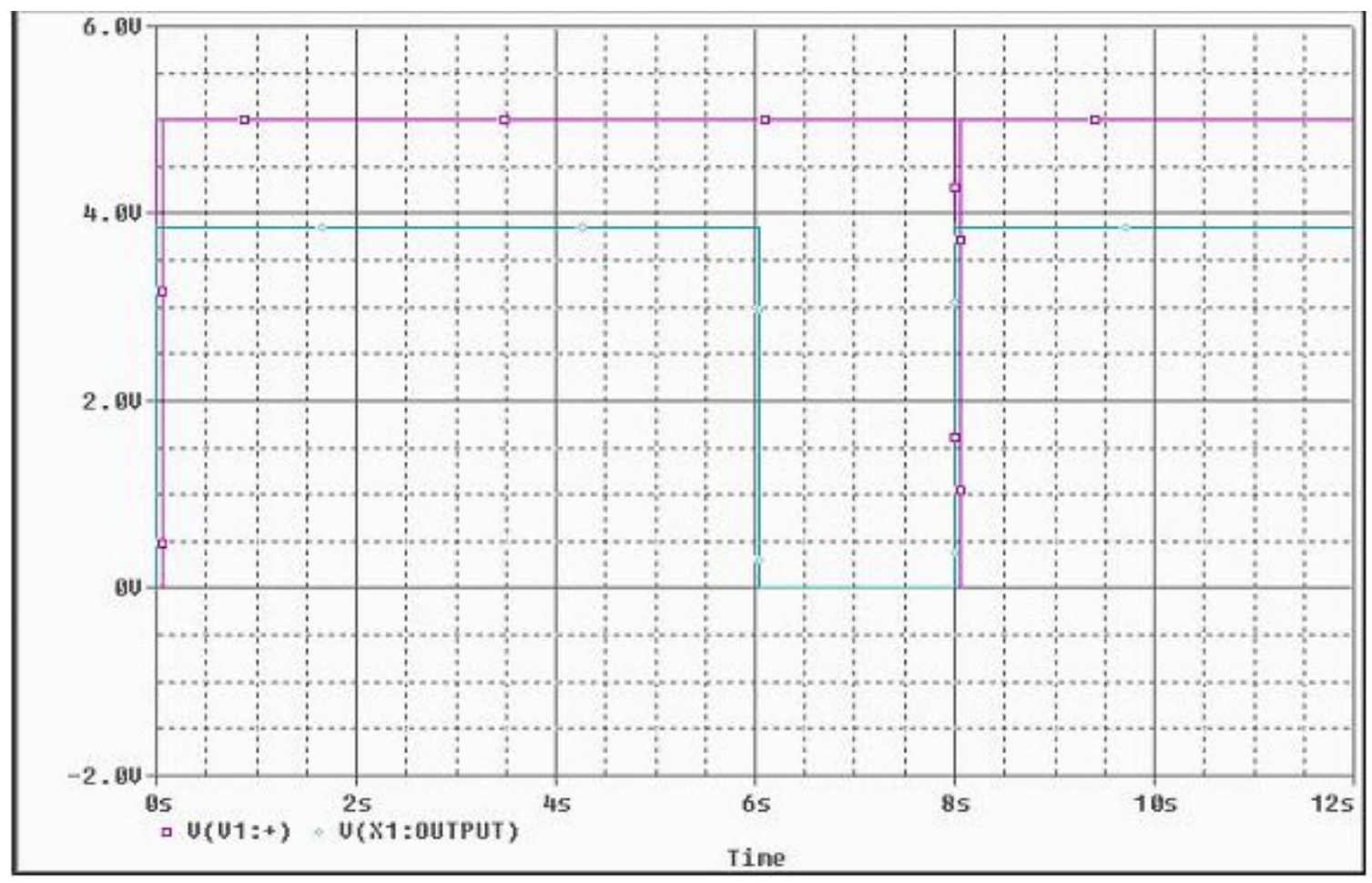

Fig.8.6 Represents: Vout (cyan, pin 3 LM555) vs Vin (magenta, pin 2 LM555)

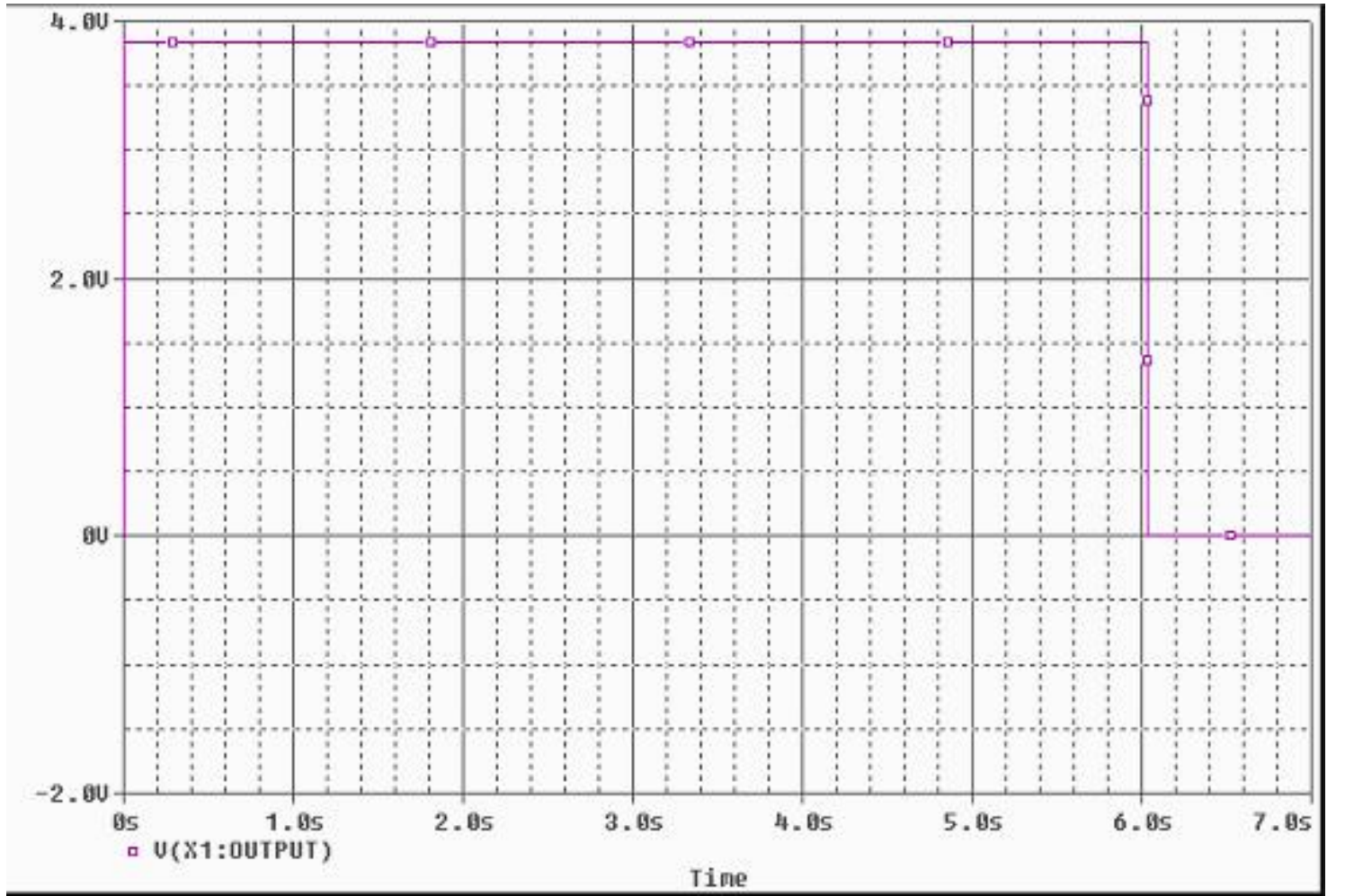

Fig.8.7 The output of the Monostable 1 (6 seconds, after the transition High to Low of the Trigger input, pin2 of LM555) 
B) Monostable 2 (25 ms, pulse buffer)

Changing the value of R2 to $24 \mathrm{~K}$ and the value of $\mathrm{C} 2$ to $10 \mu \mathrm{F}$, a width of the output pulse of $25 \mathrm{~ms}$ resulted, suitable to attack the counter without losing the narrow pulses.

Input: pin 12, SN7414

High to Low transition

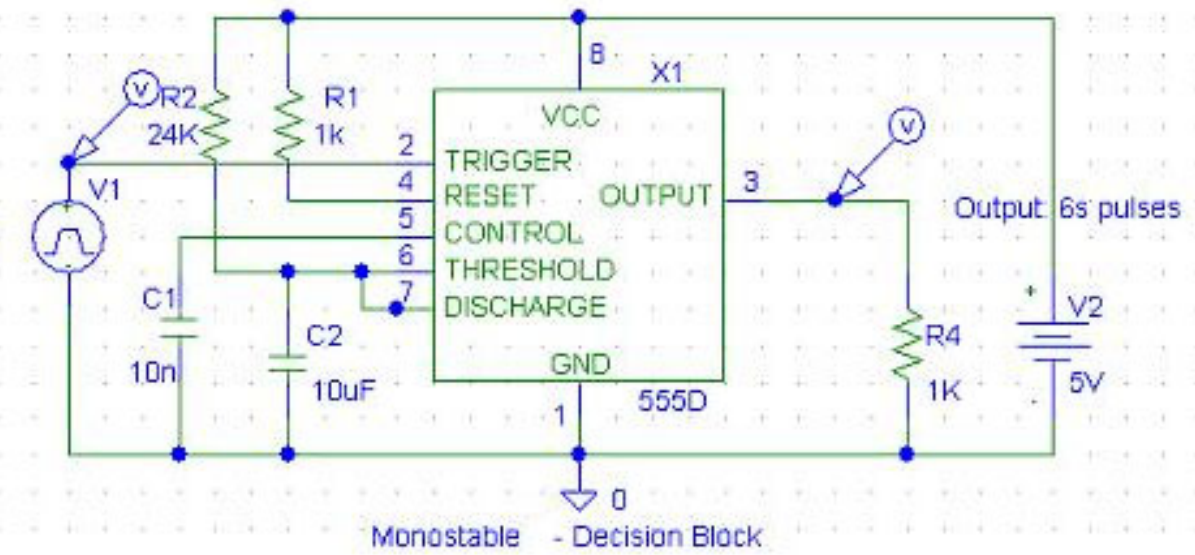

Fig.8.8 The schematic principle for Monostable2 is the same as used for Monostable1

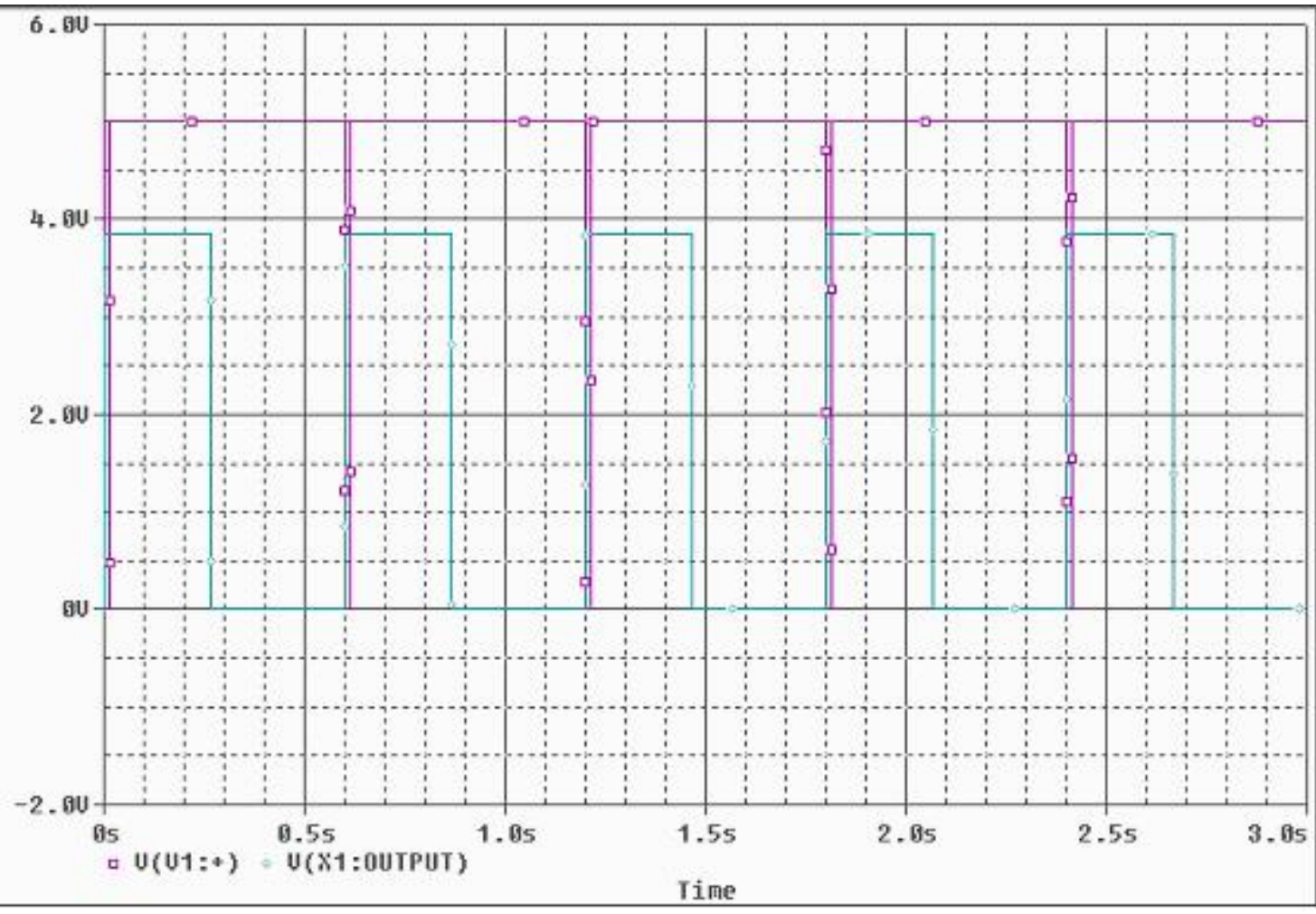

Fig.8.9 Represents Vouptut (cyan, pin3 LM555, To $=25 \mathrm{~ms}$ ) vs Vinput (magenta, pin2 LM555, Tin $=5$ to $10 \mathrm{~ms}$ ) 


\section{C) Logic and Decisional blocks simulation}

The next schematic is intuitive for understanding the Logic and Decisional blocks, together. The PIC and the LCD could not be included in the PSpice (student version) simulation, due to their complexity.

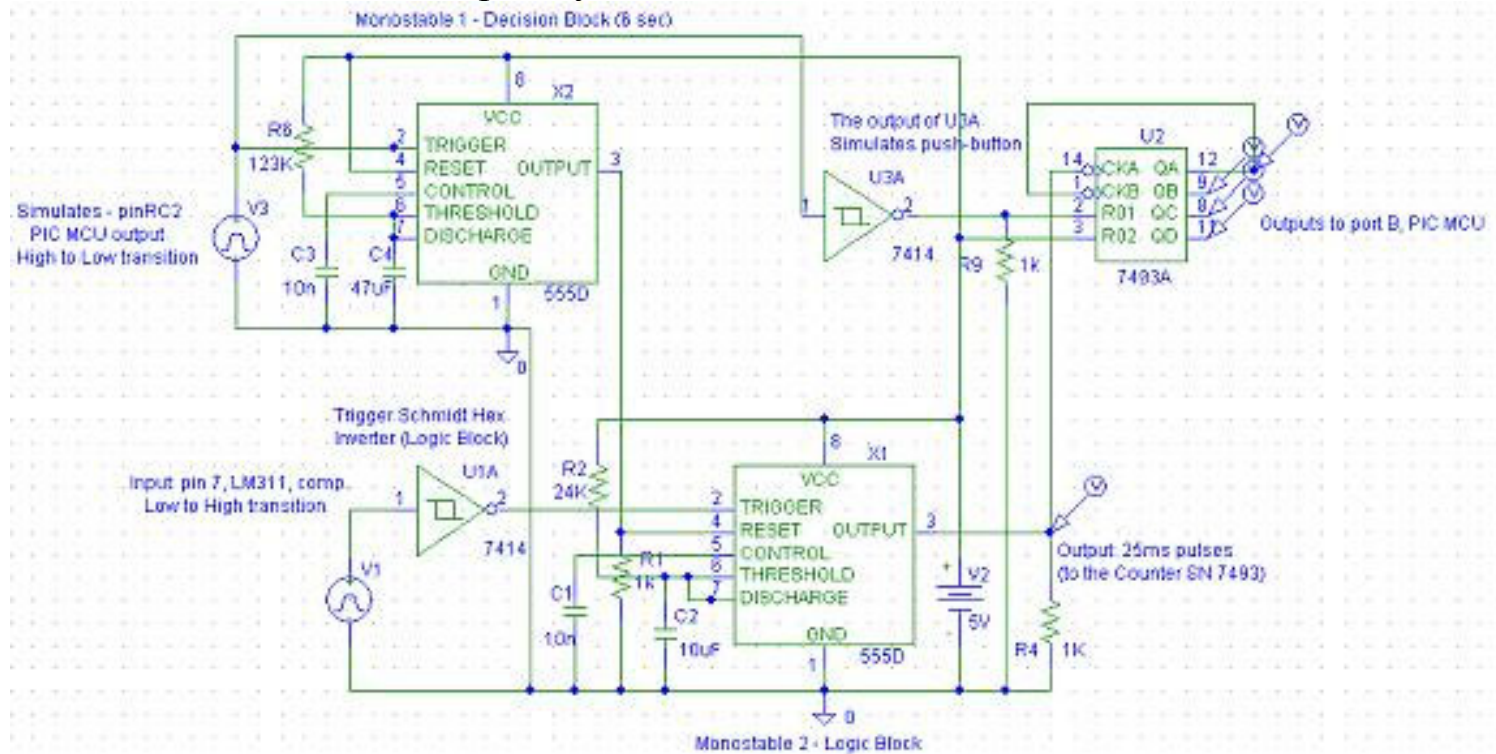

Fig.8.10 Logic and part of Decision Block interconnections (without PIC and LCD)

The simulation of the circuit in the above schematic shows the output pulses of the 7493 counter versus the input (pulses coming from the Monostable 2)

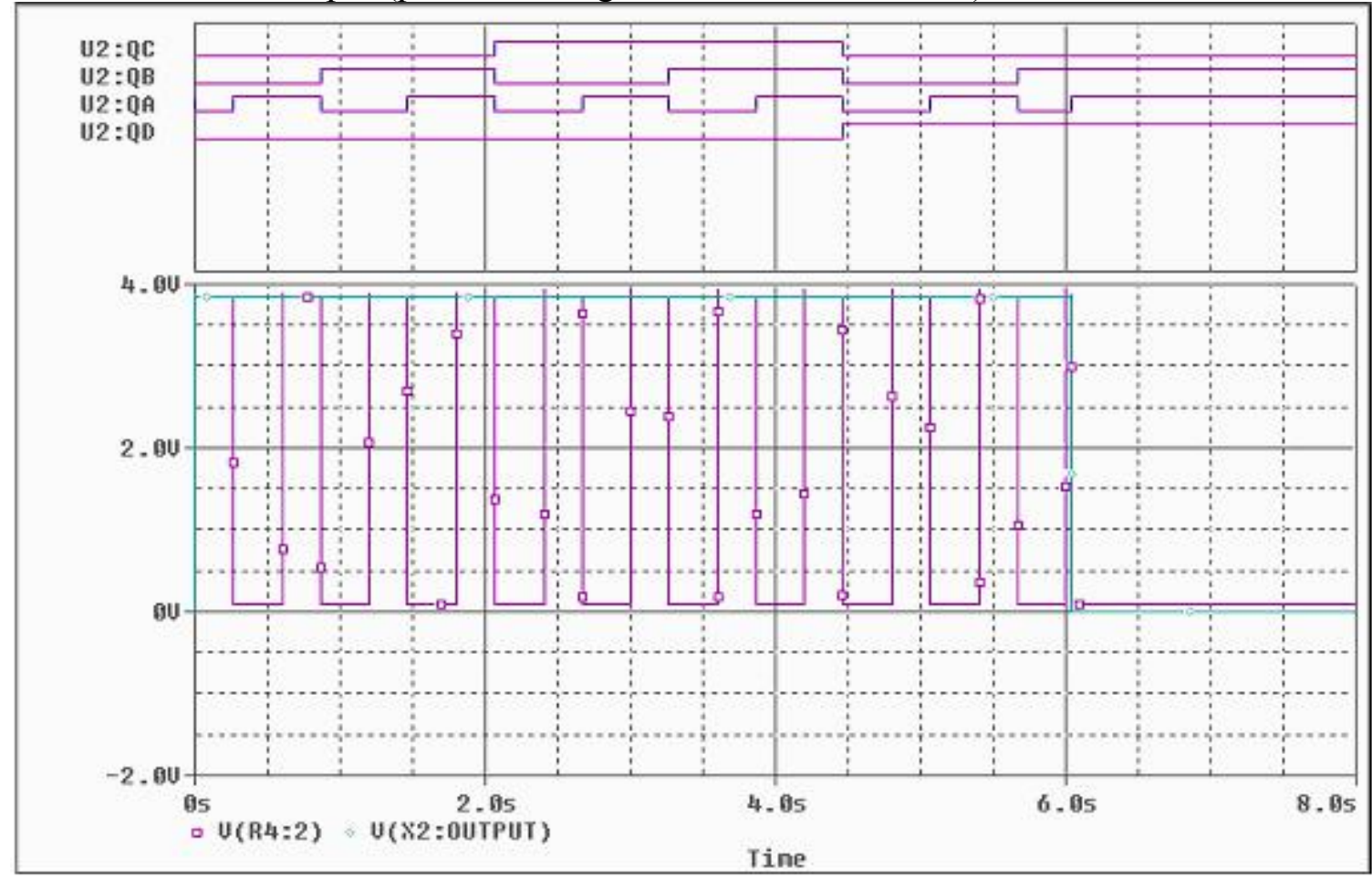

Fig.8.11 Above- the outputs from the binary Counter ( $Q A, Q B, Q C$, $Q D)$ Center - Red: output of M1 (6sec.); Green: output of M2 (10ms) 


\section{D) Real Signal showing the Monostables interaction (acquired from the Scope)}

The oscilloscope Tektronix TDS 3052B used during the research for this project permits acquiring the signals from the screen onto a floppy disk. The screen signal is acquired as a coma delimited, Microsoft Excel spreadsheet, and then through Matlab is reconverted into an image.

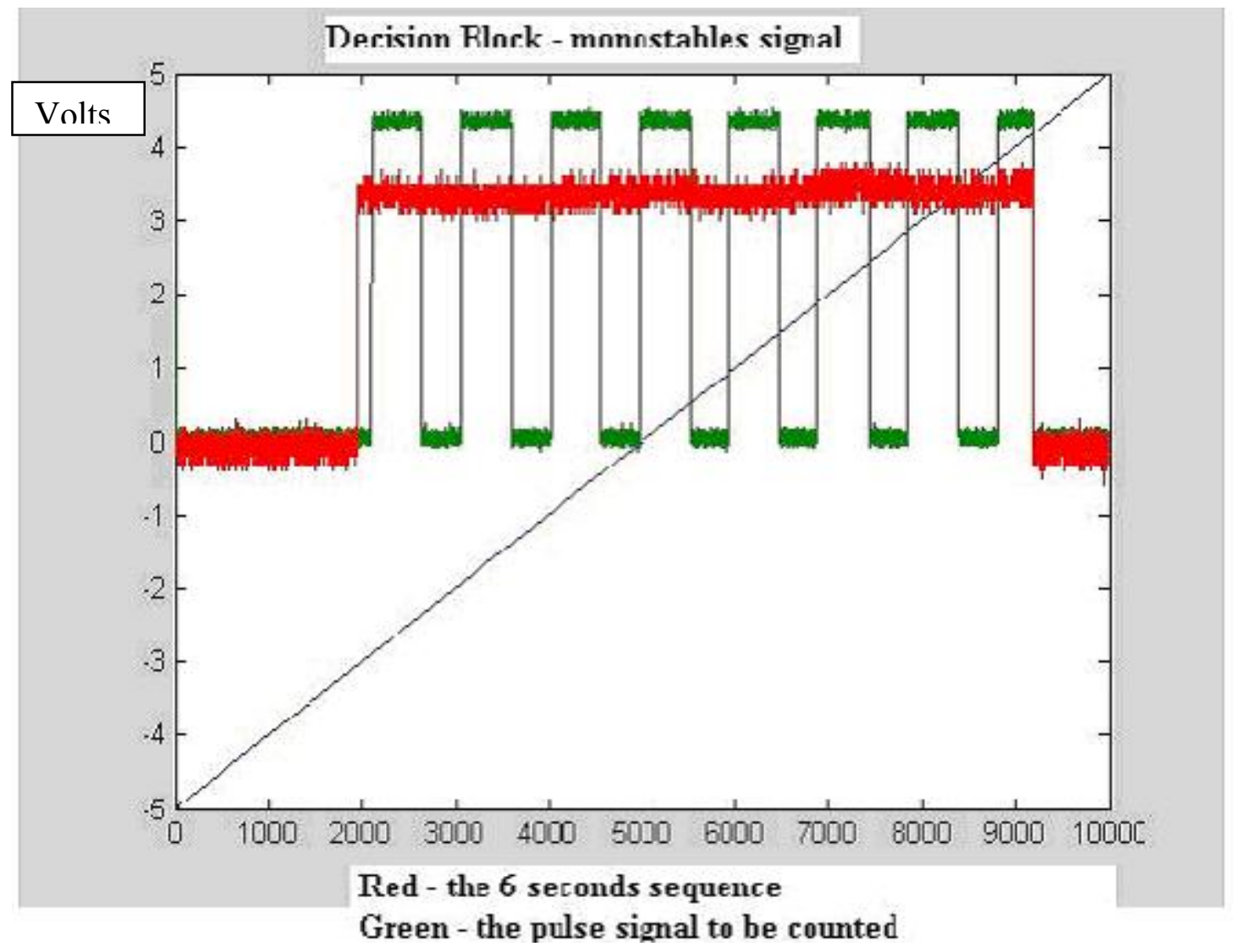

Fig.8.12 The signal output from the monostables, acquired from the Oscilloscope The oscilloscope was: Tektronix, TDS 3052B

With red color, the figure displays the 6 seconds sequence signal at the output of Monostable 1, (pin3 - LM555).

With green color, the figure displays the pulses outgoing from Monostable 2, (pin3 LM555) to the Binary Counter SN 7493.

Monostable 2 is active to buffer the pulses coming from the voltage comparator only if its reset pin is "High". If the reset pin is Lowered, the Monostable 2, freezes (become inactive). The signal that switches this logic is the 6 seconds sequence. 


\subsubsection{Flow-Chart of the software code}

The code is written in $\mathrm{C}$ language that makes it simple to write and read ( $\mathrm{C}$ (or $\mathrm{C}++$ ) language knowledge is necessary, and PIC-C compiler instructions (specific to the PIC device you are working with)).

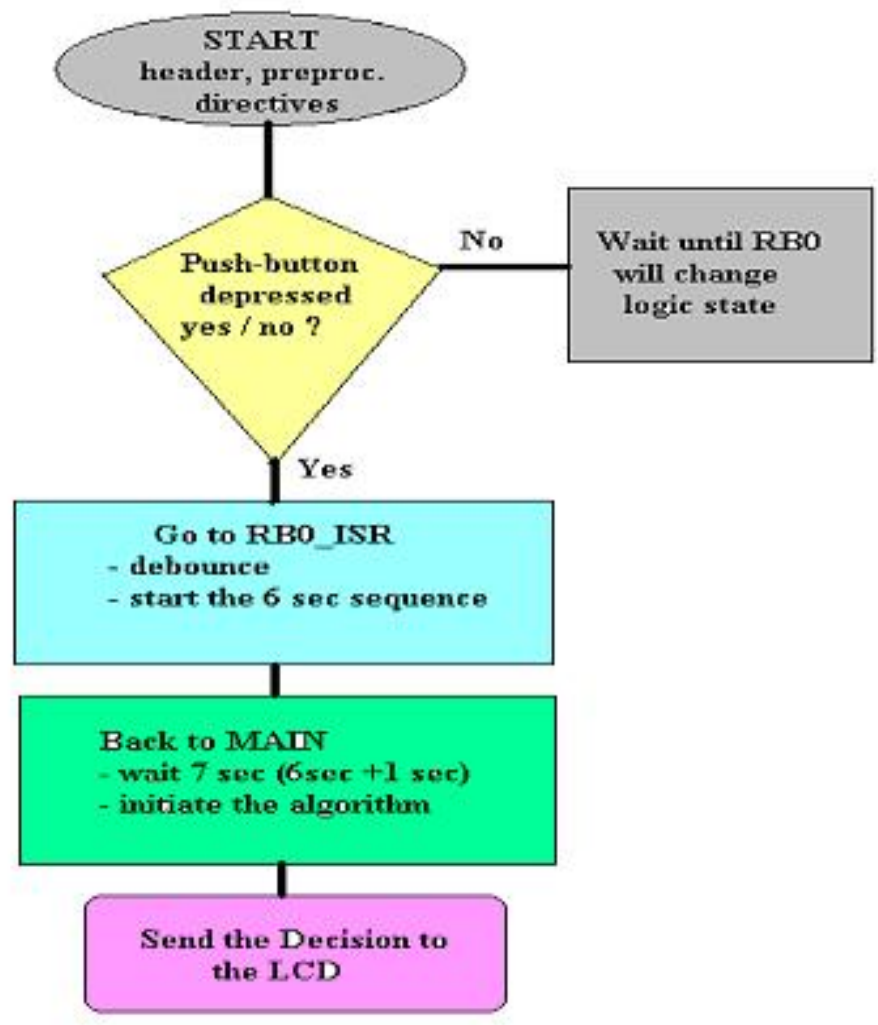

Fig.8.13 Flow-Chart for the software version 2, Decisional Block

See Appendix A.C3 for the C code.

\section{Explanation of the flow-chart and code design:}

First, the header was written, preprocessor directives and variable declarations.

Second, the function RB0_Isr that is the Interrupt Service Routine was written. This the subroutine in which the controller will jump after RB0 (pin 21, PIC18F252) changes logic because of the push-button.

The code has two branches, according with the logic state of the push-button switch:

1) If the push-button is not depressed, the system is in a wait position. The LCD is still displaying the resolution of the previous measurement.

2) If the push-button is depressed, the controller jump in the RB0_Isr (interrupt service routine). First instruction is to debounce the push-button. Then the 6 seconds sequence is started by switching from High to Low the logic status of the RC2 (pin\# 17, PIC 18F252).

When the controller goes back in Main, initiates a 7 seconds wait (the 6seconds sequence has to terminate), prior to reading and processing data acquired from the Counter outputs. Finally, the decision is sent and displayed by the LCD. 


\section{Chapter 9 FURTHER WORK}

\subsection{Sensors}

First, an analysis of efficiency should be done, in order to see if it is worthwhile to keep photo-plethysmography as the sensor principle, and not another method (for example, pressure based transduction (based on piezo-electric transducers)[7], spectrometry based sensors (Lumidigm Corp., Lumi-Sure Sensor, see www.lumidigm.com), or microwave Doppler Radar Sensing [29]).

\subsubsection{The Emitter and Detector (the photo-elements)}

\section{a) Wider area detectors}

As an improvement of the photo-elements used in the reflectance sensor, the use of bigger area detectors (thus improving the quality of the signal without raising the power in the emitter (or emitters) is recommended.

b) Wavelengths around $1.3 \mathrm{um}$ (micrometers)

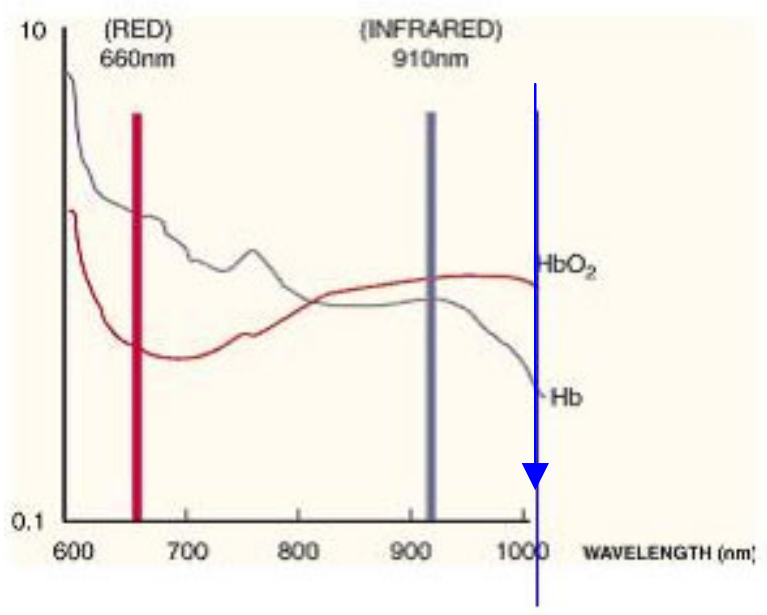

Fig.9.1 Oxyhemoglobin (red) and Deoxyhemoglobin (violet) absorbance versus wavelength of the incoming light. (660nm-red and 910nm-NIR)

As fig.9.1 shows, if one goes for longer wave-lengths, like 1.3um, (see the blue arrow in the figure) the difference between the Oxy-hemoglobin and De-oxy-hemoglobin is much bigger. It is assumed that the nature of the resulting signal is not just plethysmographic (as stated in 1.2.2, page 2 of this thesis), so the absorbance of a specific wavelength may lead to easier and more accurate detection of the ECG-like signal.

\section{Disadvantages:}

- The first disadvantage is the price. For wider detection area, higher responsivity or larger wavelength, the price will be paid. (from less than a dollar for a standard silicon NIR PIN, 940nm up to $\$ 300$ or $\$ 400$ for a non-standard detector)

- Another disadvantage is the fact that for wider detection areas, the equivalent capacitance of the junction will increase, increasing the rising time of the detector. (e.g.: for $3.2 \mathrm{~mm}^{\wedge} \mathbf{2}$, the capacitance at $0 \mathrm{~V}$ is $45 \mathrm{pF}$; but for $100 \mathrm{~mm}^{\wedge} \mathbf{2}$, the capacitance is $1550 \mathbf{p F}(!), 40$ times greater. 


\section{Examples.}

a) InGaAs Photodiodes (See [14], page191).

- Response range: from $900 \mathrm{~nm}$ to $1700 \mathrm{~nm}$. (1.3um and $1.55 \mathrm{um}$ sensitivity).

- Large area

- High Responsivity

InGaAs photodiodes are suitable for telecom and near-IR detection.

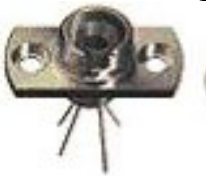

Fig.9.2 3mm $\mathrm{mm}^{2}$ photodiode. Stock $\mathrm{nr}$. NT 55-755; Area $=3 \mathrm{~mm}^{2} ;$ Price $=\$ 290.00$ [14]

The $3 \mathrm{~mm}$ photodiode is isolated in a TO-5 package with a broadband double-sided ARcoated window. With the high shunt resistance, the $3 \mathrm{~mm}$ photodiode is suitable for high sensitivity to weak signal applications.

b) Silicon detectors (see [14], page 192).

- For pulse detectors applications: fast response, biased, low capacitance, window material - borosilicate.

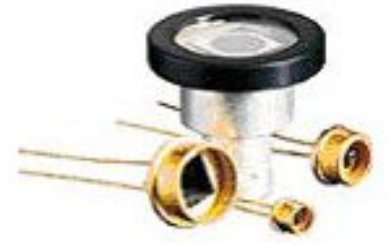

Fig.9.3 Silicon detector (from [14])

Stock nr. NT54-523; Area 15mm2; Unbiased, Low Noise; Cap. 255pF; Price $=\$ 61.00$; Stock nr. NT54-034; Area 16.4mm; Biased, Normal Resp.; Cap .330pF; Price $=\$ 33.00$;

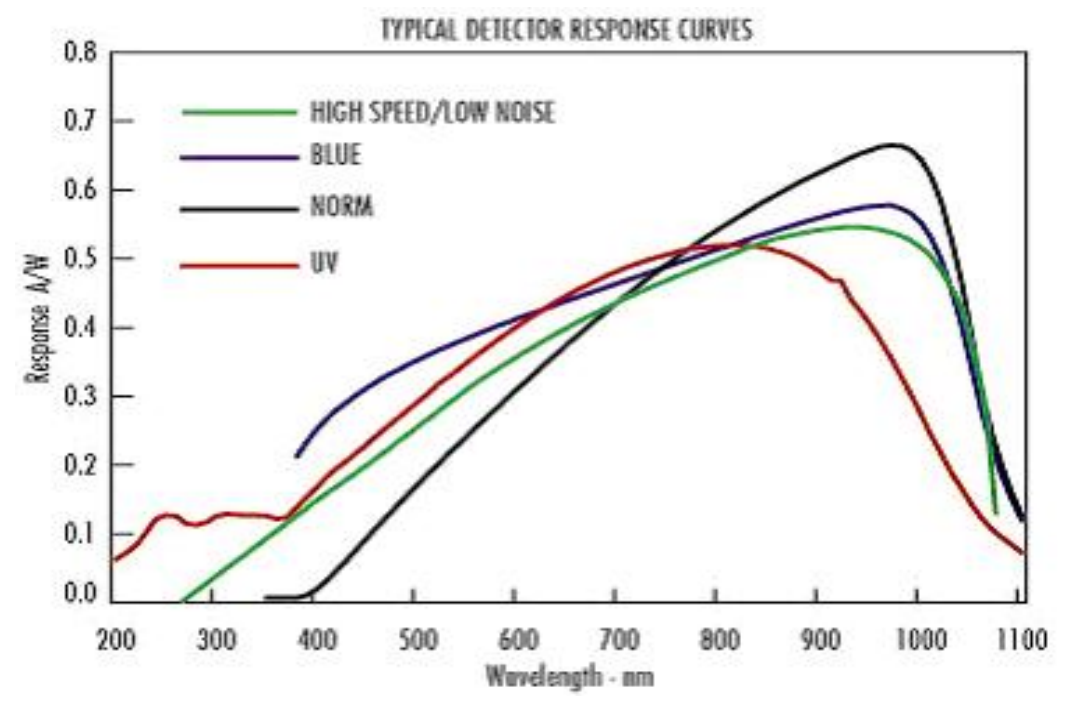

Fig.9.4 Typical response curves for Silicon Detectors (from [14])

Fig.9.4 shows that the silicon detectors (the most inexpensive yet efficient infrared detectors commercially available) can still be used for wavelengths around $1 \mu \mathrm{m}$. 


\subsubsection{The Emitter}

The first problem with the emitter is the power consumption. The strength of the signal was not improved by building a sensor with several emitters ([4], www.imagyn.com/criticalcare/pulse reflectance oximetry pg1.htm) surrounding the detector (as in Paragraph 3.9.2, fig.23). One way to still think of that is using emitters with much smaller power consumption but same IR light intensity. A search for more efficient emitters, but in the inexpensive segment of the market is recommended. Another one of the problems rising is if one tries longer wavelengths. For the research purpose, having an adjustable power and adjustable wavelength infrared emitter would be appropriate. The Multi-Channel IR Laser Light Source ([14], page nr. 188), Stock nr. H55 - 566 for 5-channel version (980nm, 1310nm, 1480nm, 1550nm, 1620nm) Stock nr. H55-867 or H55-868 - for 2-channel version, (1310nm, 1480nm respectively $1550 \mathrm{~nm}, 1620 \mathrm{~nm}$.) is recommended.

The solution to increase the signal and also the security of the system would be placing more than one sensor (by "sensor" - a pair emitter + detector is assumed) on the platen.

\subsubsection{The sensor package}

a) The sensor package has to be miniaturized to a maximum area of 8 to $10 \mathrm{~mm}$. This will allow placing two or even three sensors to work together on the platen surface, thus increasing the reliability, accuracy and security of the device. The lenses will have appropriate coating, preventing visible light from reaching the sensor. The miniaturization has to be done in a professional environment, due to the precision and small mechanical tolerances required.

b) If possible, at least the first amplifier stage should be integrated together with the sensor, thus decreasing the noise capture due to the long wires from the sensor to the analog block.

c) Optics and apertures. Research was going on using different lenses and apertures for the purpose of focusing and collimating the outgoing and incoming IR beams.

- $\quad$ apertures help in collimating, but also eliminate the side coming light or interferences.

- lenses, are 10 to 20 times more expensive than an electronic IC. They can improve the quality of the signal with up to 30 or $35 \%$. They have to be as thin as possible (because the signal is an exponential decaying function of the distance to the photo-detector. The type of the lens is singlet PCX (plano-convex). The lens is placed in between the detector and the surface material generating the incoming signal.

- $\quad$ Thus, thinner the lens, bigger the magnitude of the signal. Because of this, the lens has to have a very small focal length. 


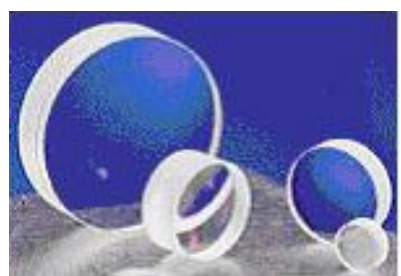

Fig.9.5 Lenses

Achromatic lenses are recommended for applications like: CCD imaging lenses for NIR (near infrared), focusing and expanding of NIR lasers and focusing or collimating lenses for fiber optics and NIR LEDs. [14].

The achromatic lenses consist of two optical components, cemented together to form an achromatic doublet, which is computer optimized to correct for on-axis spherical and chromatic aberrations. They have minimal absorption in the range 700 to $1550 \mathrm{~nm}$.

The disadvantage is the high price (around \$60.00), in comparison with calcium fluoride, the material used in this project, that costs around \$18.00.[14].

- coatings applied on the lenses surface have a filter effect for undesired wavelengths and are used to increase the transmission through an optic.

As an example of coating recommendation, from [14], page 18, is the AR coating for calcium fluoride lenses, on both sides:

\begin{tabular}{||l|l|l||}
\hline Coating code & Descript & Specifications \\
\hline 146 & NIR II[700-1550nm] & $\mathrm{R}_{\text {ave }}<1.0 \%(800-1550 \mathrm{~nm})$ \\
& LaKN22 & \\
\hline
\end{tabular}

\subsection{Analog Block}

a) The use of more efficient instrumentation amplifiers will increase the sensitivity of the block, increasing also the S/NR (signal to noise ratio), making easier the task of the Logic Block.

b) The major improvement that can be done in the Analog Block is adding an AGC loop instead of the Adjustable Resistor R8 (10K), as in the schematic on fig.9.6: 


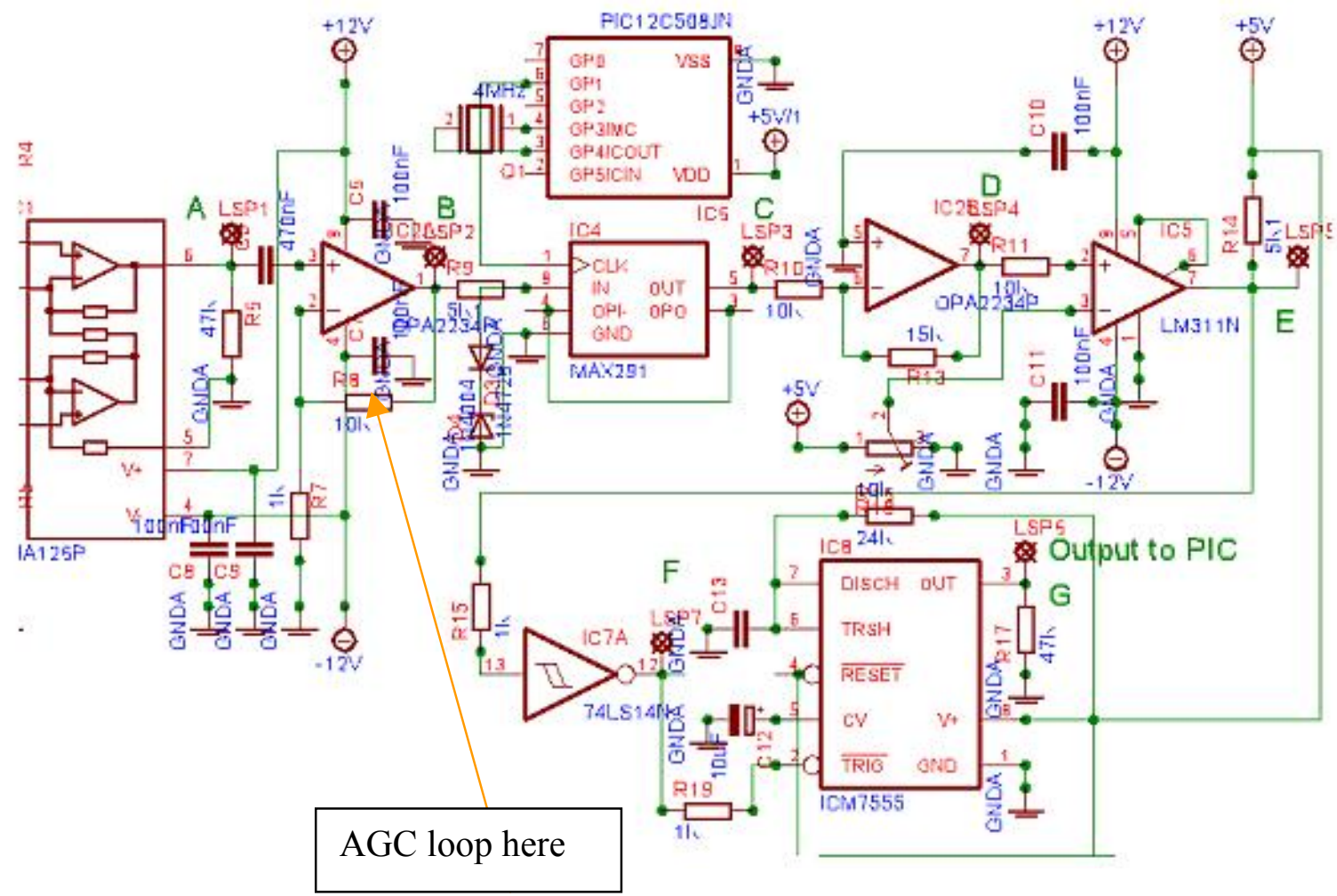

Fig.9.6 Replacing R8 (gain of IC2A-OPA2134) with a PGA (programmable gain amplifier)

\subsubsection{The AGC Loop}

a) General considerations

The AGC (Automat Gain Control) Loop is a very important part to be added in the analog block, for the design to work without any adjustments, regardless of the differences occurring due to different magnitudes of the input signal, for different people's hands. As it is specified in the Chapter 5 (Signal Processing), this project uses a comparator with fixed threshold, in contrast with some other possible methods, which are using adaptive threshold. Thus, the fact that the signal may have different levels of magnitude, from individual to individual, can lead to:

- false triggering (in the case of the magnitude being too big), or

- $\quad$ no triggering at all (in the case of the magnitude being too small)

To overcome this problem, the use of an AGC (automat gain control) loop is recommended, as in fig.9.6, instead of the adjustable resistor R8 $(10 \mathrm{~K})$, to establish the gain of the non-inverting amplifier IC2 (A), OPA 2134 (Burr Brown). The AGC loop will contain a PGA (programmable gain amplifier), a positive peak detector, and an AD/C (analog to digital converter). 


\section{b) The block diagram of the AGC loop}

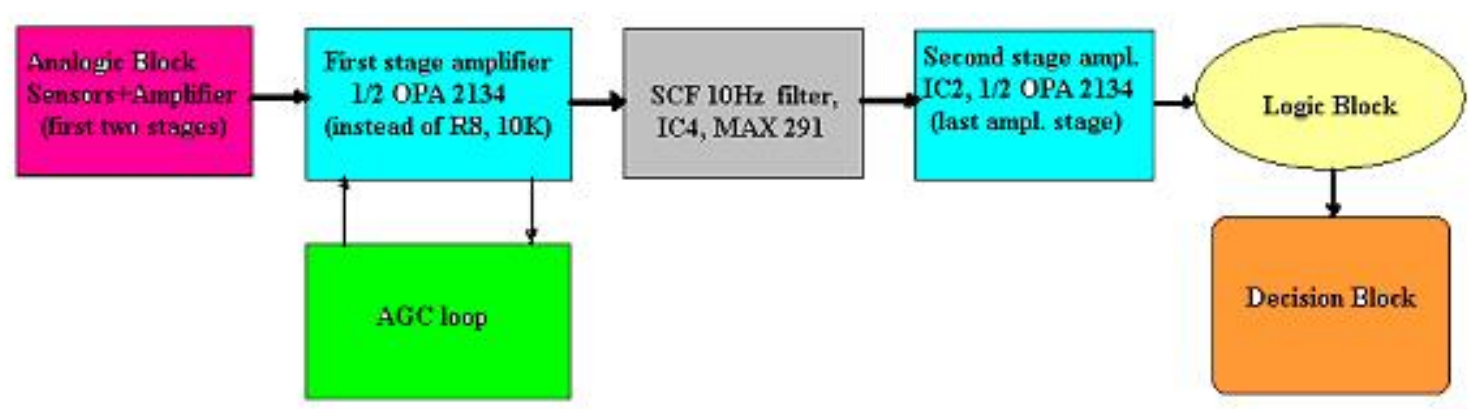

Fig.9.7 Block Diagram of the AGC loop

As it can be noticed, the AGC loop is not adjusting the gain of the last stage of the amplification chain, but the gain of the amplifier before the SCF filter. This is because the filter's (MAX 291) input doesn't support more than $+/-\mathbf{5 . 5 V}$, that is $\mathrm{Vcc}+10 \%$ and Vss $-10 \%$. So, the AGC loop was designed, such that the maximum output voltage will be:

$\operatorname{Vmax}=<\mathbf{5 . 5} \mathrm{V}$ and the minimum voltage will be: $-\mathbf{V m i n}>=\mathbf{- 5 . 5 V}$. There is a bilateral Zener limiter as a supplementary protection measure, at the input of the filter.

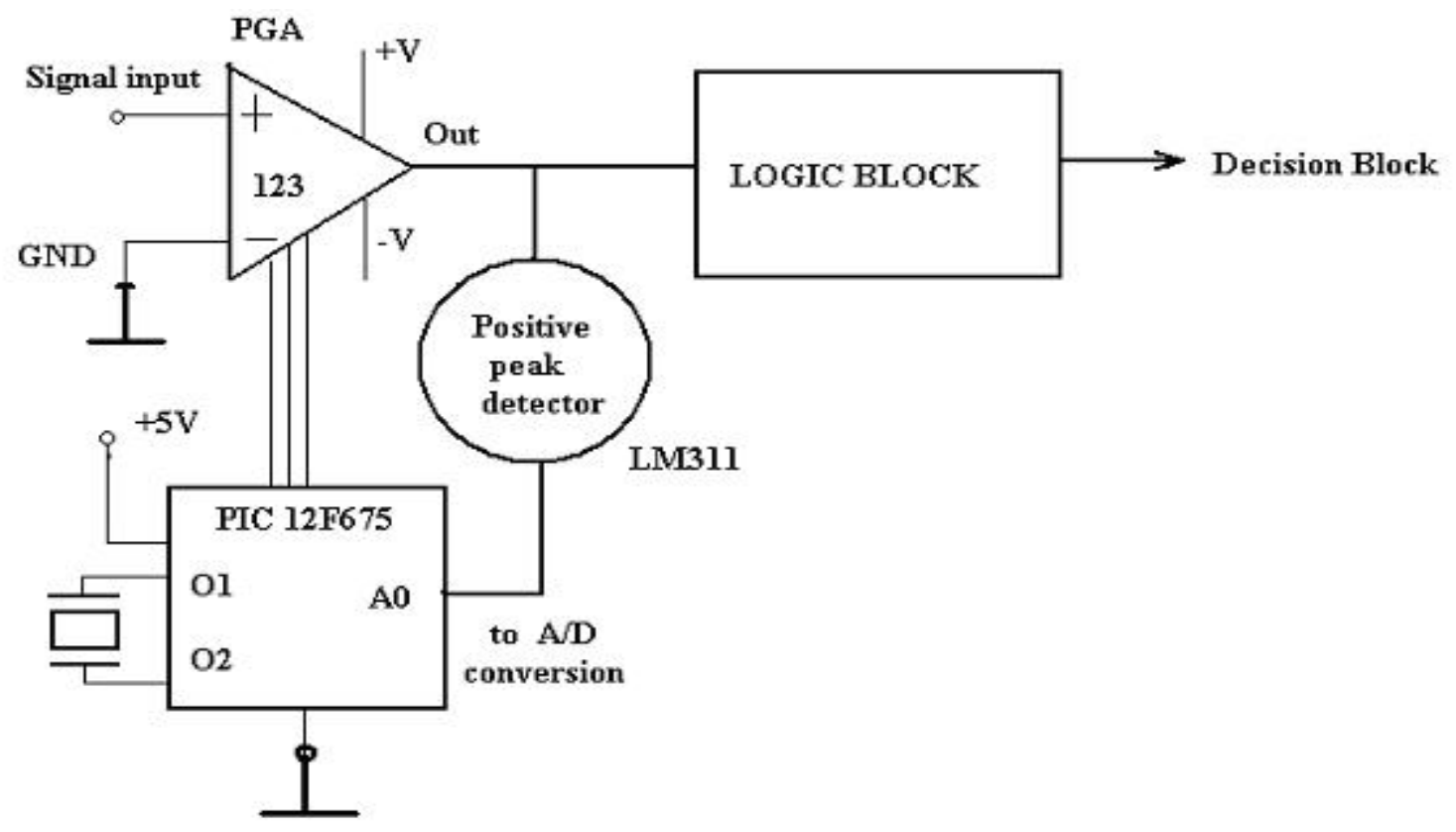

Fig.9.8 More detailed Schematic of the AGC block

The signal at the output of the amplifier is split into a positive peak-detector giving the magnitude level information to the Analog to Digital Converter. The A/DC converts the analog voltage level into a digital combination at the its output. But the output of the $\mathrm{A} / \mathrm{DC}$ is connected to the digital inputs of the PGA block. So, the PGA (programmable Gain Amplifier) will adapt its gain, according to the digital combination arriving at its digital inputs. 


\section{b) AGC loop, the positive peak detector}

Positive peak detectors (brief theory -[32], page 298,299)

The simplest peak detector can be seen in fig.9.9A, the diode rectifies and the capacitor maintains the peak. One disadvantage of this circuit is the reduction in the output voltage due to the forward voltage across the diode. The Op. Amp eliminates this disadvantage in the fig.9.9 B, because of very high gain in open loop.
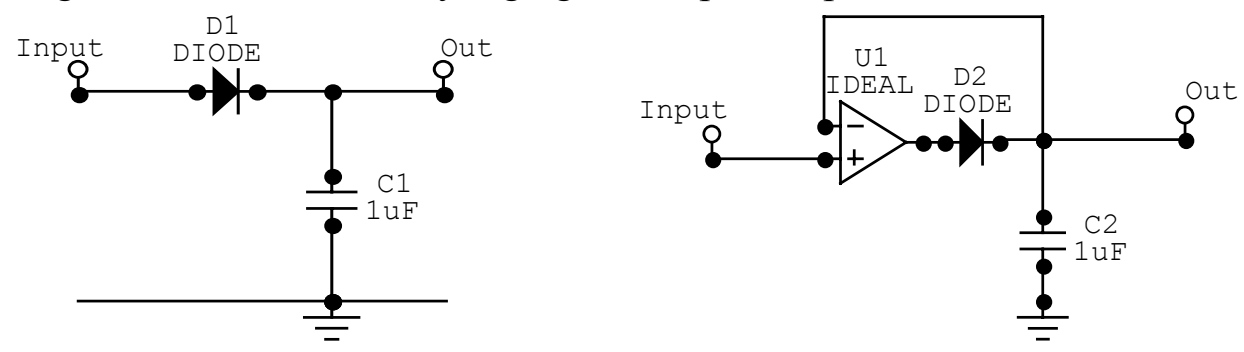

Fig.9.9.A Peak Detector with diode and capacitor, and B Peak Detector using an Op Amp.

In fig.9.9C., a voltage follower is added, with a huge input impedance, thus the load has no effect upon capacitor voltage.

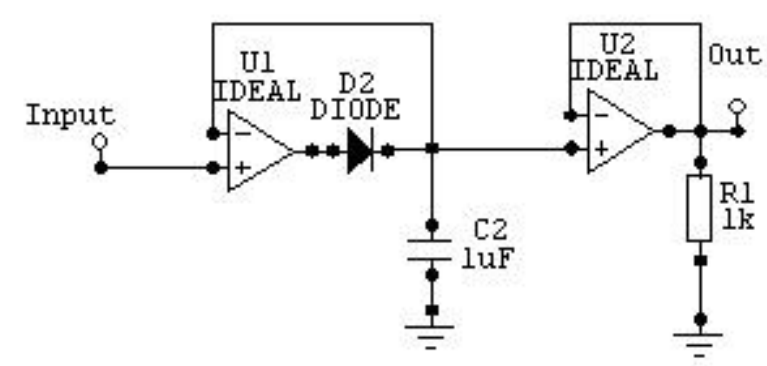

Fig.9.9.C Classic peak detector with op amps and diode (rectifier and follower)

\section{c) Practical implementation suggested}

The schematics from the above figures would work in practice, but the best thing when implementing a classical circuit is to check for an industrial pr standard application. From National Semiconductor Data Sheet of the LM 311 Voltage Comparator, $<$ www.national.com>, among other typical applications, on page 15 can be found the positive peak detector that was suggested to be used in the given application (AGC loop). 


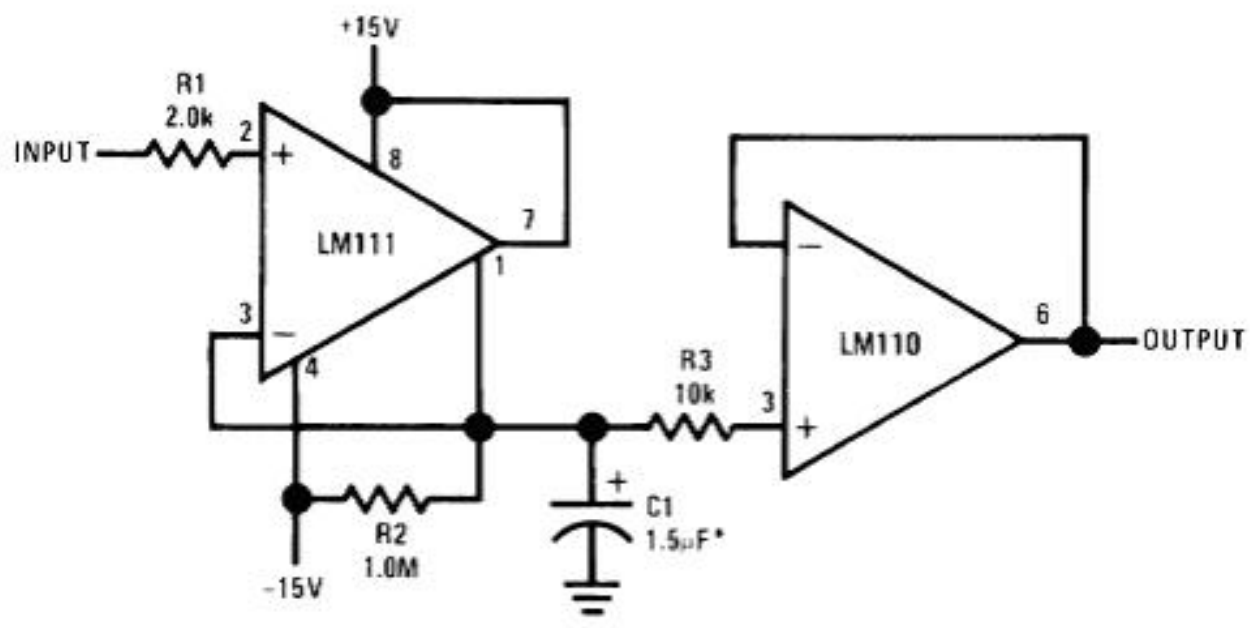

Fig.9.10 Peak Detector with LM311, typical application from National Semicond

\section{d) About PGA (Programmable Gain Amplifiers)}

From a simple variant of AGC, implemented with discrete components, to a sophisticated variant using the EI-1040 package from Electronic Innovations Corp. www.designcircuit.com, there are a lot of attractive possibilities of PGAs to be used. A research has been done among some of the most important manufacturers (National Semiconductors, Analog Devices, Microchip, Maxim, Linear Technology). The main differences between manufacturers lie in the way they control the digital inputs.

For instance, Linear Technology made an option for a small volume, low cost PGA in an 8 pin, ThinSOT package, that uses three digital input pins, as a parallel bus $(\mathrm{G} 1, \mathrm{G} 2$, G3) to control the gain of the amplifier.(see the data sheet of LTC 6910-X, from www.linear.com ). See also [19], page 70.

On the other hand, Analog Devices, Microchip, Maxim and National are using the flexible SPI serial interface to control the digital gain.

Probably, one of the most attractive, because of its simplicity and efficiency, is MCP6S21 / 22, from Microchip.( see the data sheet from www.microchip.com ). It is recommended for prototype applications in medical instrumentation, test equipment and others. This circuit can be configured for gains from $1 \mathrm{~V} / \mathrm{V}$ to $32 \mathrm{~V} / \mathrm{V}$, for single supply applications needing flexible performance. This PGA's analog functions are programmed through the SPI Interface using 16-bit words. There are two 8-bit registers to which the data is send (Instruction Register and Gain Register). These devices are initially programmed with the Instruction Register set for Nop, and the Gain Register for $+1 \mathrm{~V} / \mathrm{V}$.

As an interesting fact to mention, Analog Devices, has a family of "digital potentiometers", AD 523X, intended to replace their mechanical counterparts (the classical potentiometers), occupying lot of volume, and giving a lot of trouble because of their limited resolution, poor temperature coefficient, high resistance drift over time, and 
difficulties of remote adjustment. (For details, please check the Analog Devices Company web site, www.analog.com ). A menu of 16 instructions are providing functions such as setting the wiper position (W), storing the wiper position, incrementing / decrementing by one, incrementing / decrementing by $6 \mathrm{~dB}$ (logarithmic taper adjustment).

\subsubsection{Decisional block improvements}

After trying two versions, a roll over version and a 6 seconds sequence version, for the Decisional Block, the following improvements are suggested:

1. As a future work, the dsPICs from Microchip Technology (still under development at this time (September 2003)) would be a nice try. Through a dsPIC:

- the Logic block can be assimilated with the Decisional block, to form a single unit.

- other thresholding methods may be applied (for example, the adaptive thresholding method from [9]).

" The newest product of Microchip is the series of DSC (digital signal controllers) or "dsPIC". A dsPIC is a 16-bit modified Harvard RISC machine, combining the control advantages of a high performance 16-bit micro-controller, with the high computation speed of a fully implemented DSP (digital signal processor) to produce a single chip single instruction stream solution for embedded systems design." (from Microchip's web page: $<$ microchip.com>)

2. Upgrades working with same electronic:

- For the situation when the counter would overflow the possibility of false triggering will occur, so the following upgrade may be considered: the binary counter, SN7493, will overflow after its outputs will be all High (or logic"1"). A NAND gate with four inputs (SN74LS13) will solve the problem. Its output (pin\#6) will be connected to the reset of Monostable $2(10 \mathrm{~ms})$, that is pin\#4. The four inputs NAND gate will receive 1111 for the sixteenth pulse and after this will freeze on this last combination (since the SN74LS13 gate is resetting the Monostable, the Counter will receive no more pulses, so will freeze on the last combination). This will prevent the Counter from overflowing, giving false information to the PIC MCU.

- The C code can be improved to be more compact, and more efficient.

- Instead of the external oscillator for the 6 seconds sequence (with 555), one of the three internal timers of the PIC 18F252 can be used (for instance, the timer T0, would be suitable. It is a 16-bit register (made from two 8-bit registers, TMR0H (high) and TMR0L (low). It also has a programmable prescaler that is able to divide up to 256, if the appropriate bits (TOPS $0 / 1 / 2$ ) are selected in the T0CON register. (See pages 104 to 115 from the Data Sheet, at www.microchip.com).

- For the pulse rate reading to be more accurate - that can be improved in adjusting the 6 seconds sequence during the comparison with an accurate, professional device. 


\subsubsection{Concerning the whole hardware}

The best idea is to try to integrate the whole project into a single chip.

Research has to be done through new Integrated Circuits offers, which have embedded analog blocks (like instrumentation amplifiers) together with the MCUs.

As it was previously mentioned in paragraph 2.7, page 12, Viktor Kremin used the PSoC CY26443 from Cypress MicroSystems (see Circuit Cellar\#155, June 2003). In Crcuit Cellar \#159, October 2003, Cypress Company came out with new offers of PSoC MixedSignal Arrays with M8 Micro-controller. (PSoC is the acronym for Programmable System on-Chip device).

Cypress is mentioned, because its new PSoC is the winner of the EDN Magazine Innovation of the Year award. (EDN, like Circuit Cellar, is a Magazine for Electronic Design). The new circuits are:

CY8C27X, CY8C24X and CY8C22X. The first one is the most "expensive" - \$1.99 (!), the last one costs as low as $69 \mathrm{c}$. Among the features that can be found at www.cypress.com/ad/psoc-ceal , I would mention the CEA analog blocks (CEA = Cypress Enhanced Analog) like:

- rail to rail analog

- instrumentation amplifiers

- low voltage offset

- programmable gain amplifiers

- better stability.

\subsection{Statistical Results}

a) For the results to be considered in the real world, we have to make measurements on many people and see if the feature applies for the vast majority of them. A testing population of 50 people ([9], Results - test population, page 170), selected randomly from different ethnicity, sex and age. To be accurate in showing the results a Data Base has to be built holding all important data for this experiment, like: date of collection, temperature conditions, environmental light conditions, name, age, sex, ethnicity. The information has to be secured under PIN numbers or identification numbers, such that a further relation between names and identification numbers would be prevented.

If the percentage of people from the test population on which the experiment succeeds will be greater than $90 \%$, the idea is working and can be exploited in practice. Otherwise, new improvements have to complete the design in a manner that will facilitate the increase of the percentage to the required figure ( $90 \%$ in this case).

b) An interesting test of the liveness feature that was applied for three persons (the author, the author's wife and the author's advisor) consists of repeating the liveness detection test on the same individual 10 or 20 times to see if the hand geometry reader and the live detection feature consistently give the same accurate results for the same person. 


\section{REFERENCES}

1. Anil Jain, Ruud Bolle, Sharath Pankanti, “Biometrics - Personal Identification in a Networking Society”, Kluwer Academic Publishers, 1999, pp.1- 42 and 87-102.

2. Recognition Systems Inc., "HandKey II - Installation and Operation Manual”, Rev.2.0, www.recogsys.com .

3. SurgiVet Inc., "Veterinary Anesthesia and Monitoring Equipment” www.surgivet.com.

4. Y. Liu, J. Zhu, J. Zhu, Y. Luo, "Sensor Design of new type reflectance pulse oximetry", Optics in health care and biomedical optics; diagnostics and treatment, 15-18, Shanghai, China, IEEE, Proceedings of SPIE, vol. 4916, October 2002, pp. 98-102.

5. D. Damianou, J.A. Crowe, "Wavelength Dependence of Pulse Oximetry"Colloquium on Pulse Oximetry, Institution of Electrical Engineers, London, May, 1996.

6. NCCLS Global Consensus Guideline, "Pulse Oximetry-Proposed Guideline”-, Vol.21., 2001.

7. C. E. Davila, Ph.D., “Introduction to Biomedical Engineering EE5340”, Spring 1997, Southern Methodist University, Dallas, Texas. http://engr.smu.edu/ cd/EE5340

8. E. Hogbin, K. Stalnaker, D. Bender, C. Iga, "Finger Vitality Tester" Senior Design Project, 1999, LDCSEE at WVU (under Dr. S. Schuckers sponsorship).

9. R. McDonald, J. Jenkins, R. Arzbaecher, R. Throne; “A Software Trigger for Intra-cardiac waveform detection”, (Michigan University, Illinois Institute of Technology), 0276-6574/90/0000, IEEE, 1990, pp. 167 - 170.

10. P. A. Kyriacou, S. Powell, R. Langford, D.P. Jones, "Esophageal Pulse Oximetry Using Reflectance Photo-plethysmography”, IEEE transactions on Biomedical Engineering, vol.49, nr.11, November 2002.

11. J. Ehrenwerth, J.B. Eisenkraft; "Anesthesia Equipment, Principles and Applications" -1993 - Mosby-Year Book Inc., St. Louis, MO.

12. http://www.oximeter.org/pulseox/principles.htm 
13. R. A. Pease, "Troubleshooting Analog Circuits", EDN Series for Design Engineers, (1993, Butterworth- Heinemann.)

14. Edmund Industrial Optics - "Optics and Optical Instruments Catalog”/ 2003

15. Mike Predko, "Programming and Customizing PIC micro MCUs -; ( $2^{\text {nd }}$ edition, 2002, McGraw-Hill)

16. Microchip Technology Inc., www.microchip.com - (PIC 12F629, PIC18F452 and PIC18F252 - data sheets)

17. Fairchild Semiconductors, Standard TTL Catalog -; 1996.

18. Texas Instruments, The TTL Data Book, 1985.

19. EDN magazine - Sept. 18 / 2003, page 170 (about LTC6910-X, PGA amplifier)

20. Dr. E. Hill, Dr. M.D. Stoneham, "Practical Applications of Pulse Oximetry" Dept. of Anesthetics of Oxford Radcliff NHS Hospitals, Oxford, UK., 2000.

21. Merriam-Webster Dictionary On-Line - "The Language Center", http://www.m-w.com/home.htm

22. Course by SSB Healthcare Division, "Pulse Oximetry and Oxyhemoglobin Dissociation Curve”, ContinuingEducation.com, Inc., based in Tallahassee, FL (provider of e-education solutions to the medical community) www.continuingeducation.com/nursing/pulseox/pulseox.pdf .

23. Dr. Biswajit Daas “Optical Sensors II - UV, VIS and NIR Sensors”, Sensors and Control course, EE 493, LDCSEE, WVU, Fall 2002.

24. Kerry Lacanete, "A basic Introduction to Filters - Active, Passive and Switched Capacitor”, National Semiconductor, Application Note 779, 1991.

25. Bonnie Baker "Using Single Supply Op. Amps in Embedded Systems”, Microchip Technology, Application Note, 1999)

26. Bonnie Baker, “Operational Amplifiers Topologies and DC Specifications”, Microchip Technology, Application Note, 1999)

27. S. Mascaro, H. Asada, "Photo-plethysmograph Fingernail Sensors for Measuring Finger Forces without Haptic Obstruction”, IEEE trans. Robotics and Automation, vol.17, nr.5, October 2001. 
28. G. Rizzoni, Ph.D., "Principles and Applications of Electrical Engineering", $4^{\text {th }}$ edition, McGraw-Hill, 2000.

29. B. Lohmann, O. Boric-Lubecke, V.M. Lubecke, P.W. Ong, M.M. Soudhi; "A DSP for Doppler Radar Sensing of Vital Signs", IEEE - Engineering in Medicine and Technology, Sept. / October 2002.

30. A. Khazan, Ph.D., "Transducers and their elements", Univ. of Massachusetts, Prentice Hall, 1994).

31. T. DeMassa, Ph.D., "Electric and Electronic Devices, Circuits and Instruments”, West Publishing Co., 1989.

32. Jaakko Malmivuo, Ph.D., Ragnar Granite Institute - Biomedical Engineering Lab., Tampere Institute of Technology - Finland, http://www.ee.tut.fi/rgi/kurssit/71221/ECG-measurement/. 


\section{APPENDIX A}

\section{A.A1 The Infrared LED emitter}

(Copied from www.digikey.com )

Infrared Light Emitting Diodes

Panasonlc

\section{LN77L}

\section{GaAlAs Infrared Light Emitting Diode}

For optical control systems

Features

- High-power output, high-efficiency : $P_{0}=18 \mathrm{~mW}$ (typ.)

- Fast respouse and high-speed modulation capability : $\mathrm{f}_{\mathrm{C}}=20 \mathrm{MHz}$ (typ.)

- Wide directivity : $\theta=20$ deg. (typ.)

- Tramsparent epoxy resin package

Absolute Maximum Ratings $\left(\mathrm{Ta}=25^{\circ} \mathrm{C}\right)$

\begin{tabular}{l|c|c|c}
\hline \multicolumn{1}{c|}{ Parameter } & Symbol & Ratings & Unit \\
\hline Power dissipation & $\mathrm{P}_{\mathrm{D}}$ & 190 & $\mathrm{~mW}$ \\
\hline Forward current (DC) & $\mathrm{I}_{\mathrm{F}}$ & 100 & $\mathrm{~mA}$ \\
\hline Pulse forward current & $\mathrm{I}_{\mathrm{YP}}{ }^{\circ}$ & 1 & $\mathrm{~A}$ \\
\hline Reverse voltage (DC) & $\mathrm{V}_{\mathrm{R}}$ & 3 & $\mathrm{~V}$ \\
\hline Operatiog ambieut temyerature & $\mathrm{T}_{\text {cegr }}$ & -25 to +85 & ${ }^{\circ} \mathrm{C}$ \\
\hline Storage temperature & $\mathrm{T}_{\text {seg }}$ & -30 to +100 & ${ }^{\circ} \mathrm{C}$ \\
\hline
\end{tabular}

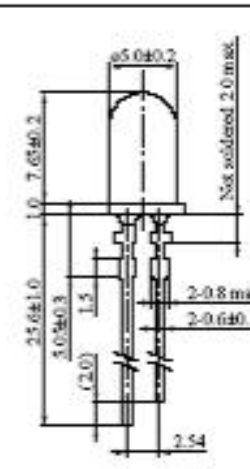

Unit : ane

$t_{N}=10 \mu 15$. Duty cycle $=0.1 \%$

Electro-Optical Characteristics $\left(\mathrm{Ta}=25^{\circ} \mathrm{C}\right.$ )

\begin{tabular}{|c|c|c|c|c|c|c|}
\hline Parameter & Symbol & Conditions & $\min$ & typ & $\max$ & Unit \\
\hline Radiant power & $P_{0}$ & $\mathrm{I}_{F}=50 \mathrm{~mA}$ & 10 & 18 & & $\mathrm{~mW}$ \\
\hline Peak emission wavelength & $\lambda_{p}$ & $I_{F}=50 \mathrm{~mA}$ & & 860 & & $\operatorname{nm}$ \\
\hline Spectral half band width & $\Delta \lambda$ & $\mathrm{I}_{F}=50 \mathrm{~mA}$ & & 40 & & un \\
\hline Forward voltage (DC) & $\mathrm{V}_{\mathrm{F}}$ & $\mathrm{I}_{\mathrm{f}}=100 \mathrm{~mA}$ & & 1.6 & 1.9 & $\mathrm{~V}$ \\
\hline Reverse current (DC) & $\mathrm{I}_{\mathrm{R}}$ & $\mathrm{V}_{\mathrm{R}}=3 \mathrm{~V}$ & & & 10 & $\mu \mathrm{A}$ \\
\hline Half-power angle & $\theta$ & The angle in which nofint intensity is 596 & & 20 & & deg. \\
\hline Cutoff frequency & $f_{c}^{*}$ & $\mathrm{I}_{\mathrm{pp}}=50 \mathrm{~mA}+10 \mathrm{~mA} \mathrm{pp}_{\mathrm{pp}}$ & & 20 & & $\mathrm{MHz}$ \\
\hline
\end{tabular}




\section{A.A2 Detector - PIN Photodiode, PNZ 334 (Panasonic)}

(From the web site: www.digikey.com )

PIN Photodiodes

Panasonlc

\section{PNZ334 (PN334)}

PIN Photodiode

For optical fiber communication systems

Features

- Plastic type package (o 5)

- High coupling capability suitable for plastic fiber

- High quastum efficiency

- High-speed response

Absolute Maximum Ratings $\left(\mathrm{Ta}=25^{\circ} \mathrm{C}\right)$

\begin{tabular}{|c|c|c|c|}
\hline Parameter & Symbol & Ratings & Unit \\
\hline Reverse voluge (DC) & $V_{R}$ & 30 & $\mathrm{v}$ \\
\hline Power dissipation & $P_{0}$ & 100 & $\mathrm{nnW}$ \\
\hline Operating antient tempenture & $\mathrm{T}_{\mathrm{ag}}$ & $-25 t 0+85$ & ${ }^{\circ} \mathrm{C}$ \\
\hline Stornge temperature & $T_{d x}$ & -30 to +100 & "C \\
\hline
\end{tabular}

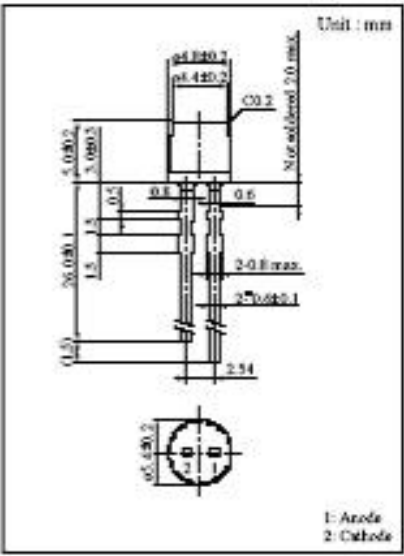

Dimensions of detection area

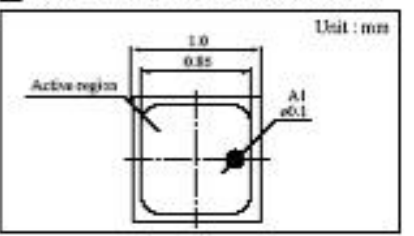

Electro-Optical Characteristics $\left(\mathrm{Ta}=25^{\mathrm{I}} \mathrm{C}\right)$

\begin{tabular}{|c|c|c|c|c|c|c|}
\hline Parameter & Symbol & Conditions & $\min$ & typ & $\max$ & Unit \\
\hline Dark current & $I_{0}$ & $\mathrm{~V}_{2}=10 \mathrm{~V}$ & & 0.1 & 10 & nA \\
\hline Photo current & I. & $\mathrm{V}_{\mathrm{g}}=10 \mathrm{~V}, \mathrm{~L}=1000 \mathrm{l \textrm {s } ^ { * }}$ & 5 & 7 & & $\mu \mathrm{A}$ \\
\hline Peak semsitivity waneleugib & $\lambda_{\mathrm{p}}$ & $\mathrm{V}_{\mathrm{R}}=10 \mathrm{~V}$ & & 850 & & $\mathrm{~nm}$ \\
\hline Response time & $4,4^{2}$ & $\mathrm{~V}_{\mathrm{R}}=10 \mathrm{~V}, \mathrm{R}_{\mathrm{L}}=50 \mathrm{\Omega}$ & & 2 & & $\mathrm{ab}$ \\
\hline Capacitance between pins & $\mathrm{C}_{1}$ & $\mathrm{~V}_{\mathrm{e}}=0 \mathrm{~V}, \mathrm{f}=1 \mathrm{MHz}$ & & 6 & & $\mathrm{pF}$ \\
\hline Acceptance half angle & $\theta$ & 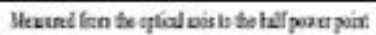 & & 70 & & $\operatorname{deg}$. \\
\hline
\end{tabular}

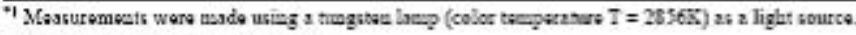

"2 Swiocling time massuromeat circuet

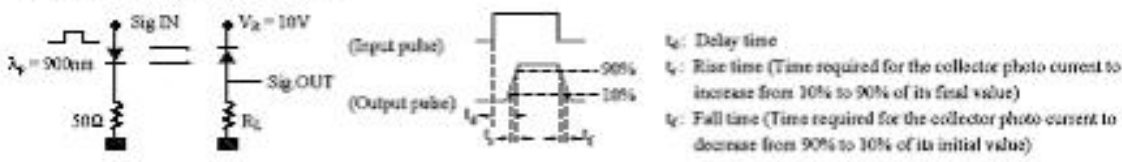




\title{
A.A3 Emitter: Plastic infrared LED - Fairchild Semiconductor
} Copied from www.digikey.com .

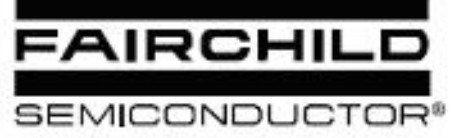

\author{
PLASTIC INFRARED \\ LIGHT EMITTING DIODE
}

\section{QED422 QED423}
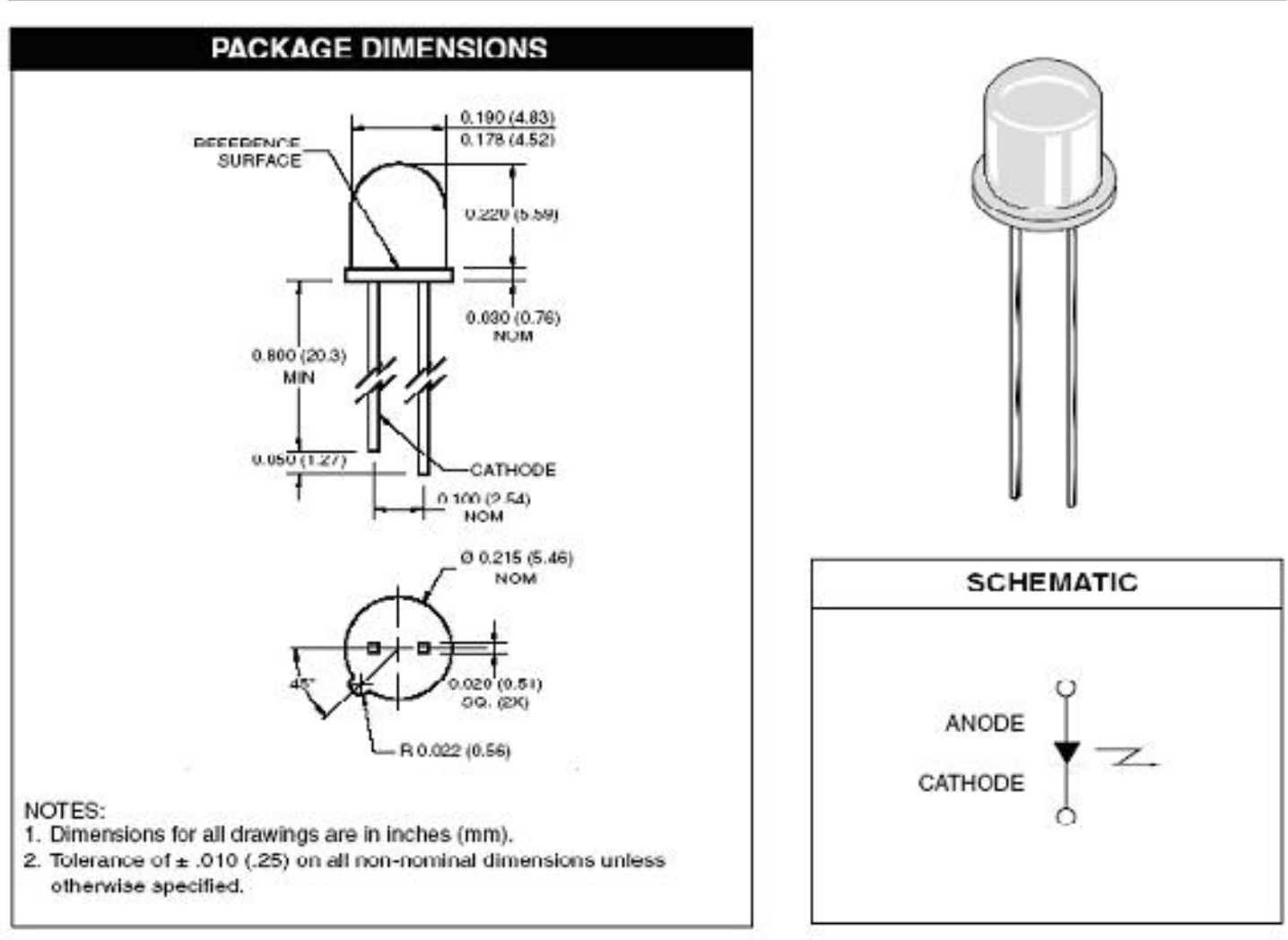

\section{DESCRIPTION}

The OED422/423 is an 880 inn AlGaAs LED encapsulated in a clear, purple tinted, plaslic TO-46 packaye.

\section{FEATURES}

- $x=880 \mathrm{mII}$

- Chip material = AIGaAs

- Package type. Plastic TO-46

- Matched Photosensor: QSD722/723/724

- Medium Wide Emisaion Anglo, $30^{\circ}$

- High Output Power

- Package material and color: clear, purple tinted, plastic 


\section{A.A4 Reflective photo sensor from Panasonic. CNB 1303 (From www.digikey.com )}

\section{Reflective Photosensors (Photo Reflectors) Panasonic}

\section{CNB1303}

\section{Reflective Photosensor}

Overview

CNB1303 is a small, thin reflective photosensor consisting of a bight efficiency GaAs infrared light enitting diode which is inlegrated with a high sensitivity Si pbototransistor in a single resin package.

Features

- Ultramimature, this type : $2.7 \times 3.4 \mathrm{~mm}$ (teright: $1.5 \mathrm{~mm}$ )

- Visible light cutoft resis is used

- Fast response : $t_{y}, t_{\mathrm{f}}=20 \mathrm{uss}$ (typ)

- Easy interface for control circuit

Applications

- Control of motor and other rotary tuits

- Detection of position and edge

- Detection of paper, film and cloch

- Start, end mark detection of maguteric tape

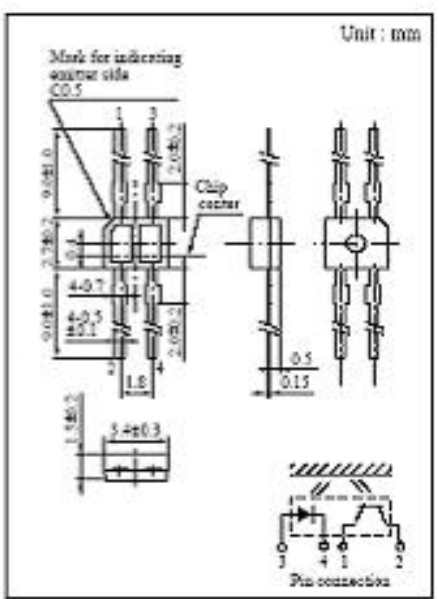

Absolute Maximum Ratings $\left(\mathrm{Ta}_{\mathrm{a}}=25^{\circ} \mathrm{C}\right.$ )

\begin{tabular}{|c|c|c|c|c|}
\hline \multicolumn{2}{|r|}{ Parameter } & Simio & Ratings & Unit \\
\hline \multirow{3}{*}{$\begin{array}{l}\text { Impt (lightt } \\
\text { emitting diode) }\end{array}$} & Reverse wollage (DC) & $v_{2}$ & 3 & $\mathrm{~V}$ \\
\hline & Forvard current (DC) & $\mathrm{I}_{F}$ & 50 & mit \\
\hline & Power dissipation & $P_{0}^{n}$ & 75 & $\mathbb{w W}$ \\
\hline \multirow{4}{*}{$\begin{array}{l}\text { Output (Photo } \\
\text { transistor) }\end{array}$} & Collector curreat & $\mathrm{I}_{c}$ & 20 & mA \\
\hline & Collector to enitter velinge & $V_{C 8 O}$ & 30 & v \\
\hline & Emalter to collector volage & $V_{B C O}$ & 5 & $\mathrm{v}$ \\
\hline & Collector power dirssipatioo & $P_{c}^{n}$ & 50 & $\mathrm{mWW}$ \\
\hline \multirow{2}{*}{ Temperante } & 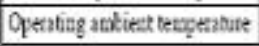 & $T_{g p r}$ & $-2510+85$ & $\mathrm{C}$ \\
\hline & Storage temperature & $\mathrm{T}_{\text {atr }}$ & -30 to +100 & 'c \\
\hline
\end{tabular}

\footnotetext{
${ }^{1}$ Input power derating satio is

$1.0 \mathrm{~m} \mathbb{W}^{\prime} \mathrm{C}$ at $\mathrm{Ta} \geq 25^{\circ} \mathrm{C}$.

2 Output power desating tatio is 0.67 wW/ $\mathrm{C}$ at $\mathrm{Ta} \geq 25^{\circ} \mathrm{C}$.
}

Electrical Characteristics $\left(\mathrm{Ta}=25^{\circ} \mathrm{C}\right)$

\begin{tabular}{|c|c|c|c|c|c|c|c|c|c|}
\hline \multicolumn{4}{|c|}{ Parameter } & Symbol & Condilions & $\min$ & typ & $\max$ & Unit \\
\hline \multirow{3}{*}{$\begin{array}{l}\text { Inpeut } \\
\text { chax:mitis: }\end{array}$} & \multicolumn{3}{|c|}{ Forwasd voltage (DC) } & $V_{r}$ & $I_{\mathrm{g}}=50 \mathrm{~mA}$ & & 1.3 & 1.5 & $\mathrm{v}$ \\
\hline & \multicolumn{3}{|c|}{ Reverse current (DC) } & $\mathrm{Ig}_{\mathrm{g}}$ & $V_{2}=3 V$ & & 0.01 & 10 & $M A$ \\
\hline & \multicolumn{3}{|c|}{ Capacitunce beween terminals } & $\mathrm{C}_{\mathrm{n}}$ & $\mathrm{V}_{\mathrm{g}}=0 \mathrm{~V}, \mathrm{f}=1 \mathrm{MHz}$ & & 30 & & $\mathrm{p}$ \\
\hline Apytatiós & \multicolumn{3}{|c|}{ Collector cutoff current } & Eeo & $V_{c z}=10 \mathrm{~N}$ & & & 200 & nA \\
\hline \multirow{4}{*}{$\begin{array}{l}\text { Trusfet } \\
\text { chankritios }\end{array}$} & \multicolumn{3}{|c|}{ Collector current } & $\mathrm{IC}^{+\mathrm{L} .2}$ & 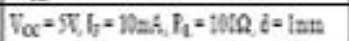 & 90 & & 880 & $\mathrm{HA}$ \\
\hline & \multicolumn{3}{|c|}{ Leakage current } & $I_{D}$ & $V_{c c}=5 \mathrm{~V}, \mathrm{lg}=10 \mathrm{ad}, \mathrm{R}_{\mathrm{L}}=100 \Omega$ & & & 200 & $n A$ \\
\hline & \multicolumn{3}{|c|}{ Respouse time } & $i^{* 5}, t^{4}$ & $v_{c c}=5 \mathrm{~V}, 1_{c}=0.1 \mathrm{~mA}, \mathrm{P}_{\mathrm{L}}=100 \Omega$ & & 20 & & $\mu \mathrm{s}$ \\
\hline & \multicolumn{3}{|c|}{ Collacor to exizer sanyassa volonge } & $\mathrm{V}_{\text {caus }}$ & $\mathrm{I}_{\mathrm{r}}=20 \mathrm{~m} \mathrm{~A}, \mathrm{I} \mathrm{c}=0 . \mathrm{Im} \mathrm{A}$ & & & 0.4 & $\mathrm{v}$ \\
\hline \multicolumn{10}{|c|}{ "I ${ }^{1}$ clarsifications } \\
\hline \multicolumn{2}{|c|}{ Class } & Q & \multicolumn{2}{|l|}{ R } & 5 & & & & orstod $A$ \\
\hline \multicolumn{2}{|c|}{$1_{c}(\mu A)$} & 90 to 220 & \multicolumn{2}{|c|}{15050440} & 350 to 580 & & $\sqrt{2}$ & & \\
\hline
\end{tabular}




\section{A.A5 Voltage amplifier, micro-power instrumentation amplifier Burr-Brown INA126 (copied from www.digikey.com )}
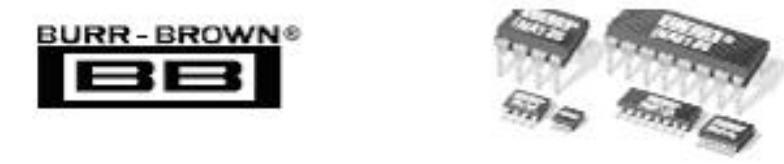

INA126

INA2126

MicroPOWER INSTRUMENTATION AMPLIFIER Single and Dual Versions

\section{FEATURES}

- LOW QUIESCENT CURRENT: $175 \mu A$ chan.

- WIDE SUPPLY RANGE: $\pm 1.35 \mathrm{~V}$ to $\pm 18 \mathrm{~V}$

- LOW OFFSET VOLTAGE: $250 \mu \mathrm{V}$ max

- LOW OFFSET DRIFT: $3 \mu V^{\circ} \mathrm{C}$ max

- LOW NOISE: $35 \mathrm{nV} / \sqrt{\mathrm{Hz}}$

- LOW INPUT BIAS CURRENT: 25nA max

- 8-PIN DIP, SO-8, MSOP-8 SURFACE-MOUNT DUAL: $16-P$ in DIP, SO-16, SSOP-16

\section{APPLICATIONS}

- industrial sensor amplifier. Bridge, RTD, Thermocouple

- PHYSIOLOGICAL AMPLIFIER: ECG, EEG, EMG

- multi-ChanNel data acquisition

- PORTABLE, BATTERY OPERATED SYSTEMS

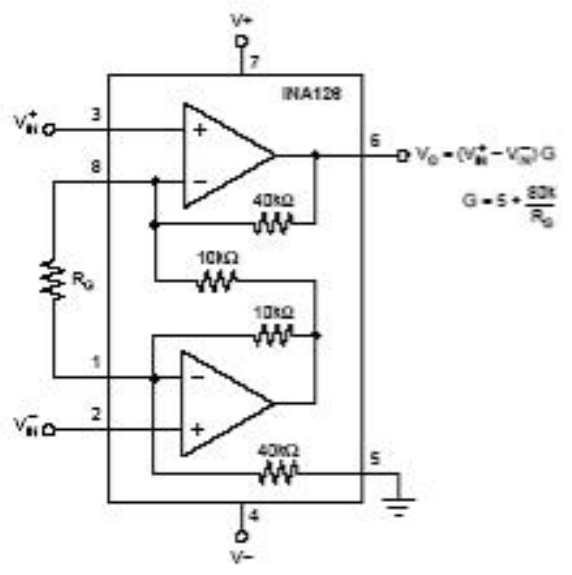

\section{DESCRIPTION}

The DNA126 and DN2126 se preision instrumersation snplifiers for accurate, low naise differential sigual scopisitisn. Their woop-sanp design prorides excellent perfor-

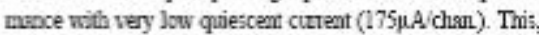
cecibined with wide operating voltage tange of $\pm 1.35 \mathrm{~V}$ to $\pm 18 \mathrm{~V}$, wakes them ideal for portable instumentstion and data scquisition systems.

Gain can be set from 5 VN to $10000 \mathrm{~V} N$ with a single extemal retistor. Laser trimmed input circuitry provides

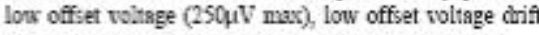
$\left(3 \mu V^{\circ} \mathrm{C}\right.$ max) snd excellent common-mode rejection Single versice package options inchlde 8-pin plastic DP, SO-8 sufface mount, and fine-pitch MSOP-8 surface-mount Dod version is arailsble in the space-ssving SSOP-16 fine. pitch surfice mount, SO-16, and 16-pin DIP. All are specified for the $-40^{\circ} \mathrm{C}$ to $+85^{\circ} \mathrm{C}$ indsistial temperanue range.

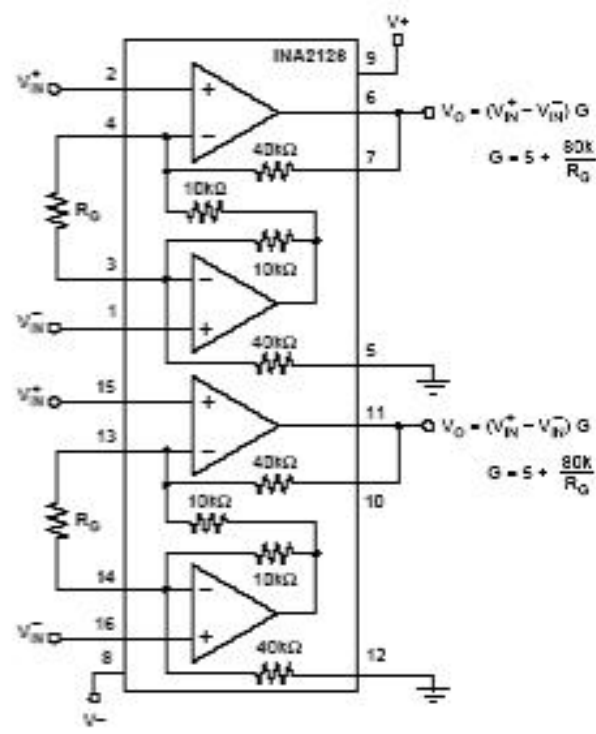




\section{A.A6 LCD - from Optrex DMC 20434 - 20 characters X 4 lines display}

(From www.optrex.com)

\section{1/O Terminal}

\subsection{Pin Assignment}

\begin{tabular}{|c|c|c|l|}
\hline No. & Symbol & Level & \multicolumn{1}{|c|}{ Function } \\
\hline 1 & VSS & - & Power Supply (OV, GND) \\
\hline 2 & VCC & - & Power Supply for Logic \\
\hline 3 & VgE & - & Power Supply for LCD Drive \\
\hline 4 & RS & H / L & Register Select Signal \\
\hline 5 & R $/$ & H / L & Read/Write Select Signal H : Read L : Write \\
\hline 6 & E & H / L & Enable Signal (No pull-up Resister) \\
\hline 7 & DB0 & H / L & Data Bus Line / Non-connection at 4-bit operation \\
\hline 8 & DB1 & H / L & Data Bus Line / Non-connection at 4-bit operation \\
\hline 9 & DB2 & H / L & Data Bus Line / Non-connection at 4-bit operation \\
\hline 10 & DB3 & H / L & Data Bus Line / Non-connection at 4-bit operation \\
\hline 11 & DB4 & H / L & Data Bus Line \\
\hline 12 & DB5 & H / L & Data Bus Line \\
\hline 13 & DB6 & H / L & Data Bus Line \\
\hline 14 & DB7 & H / L & Data Bus Line \\
\hline
\end{tabular}

\subsection{Example of Power Supply}

It is recommended to apply a potentiometer for the contrast adjust due to the tolerance of the driving voltage and its temperature dependence.

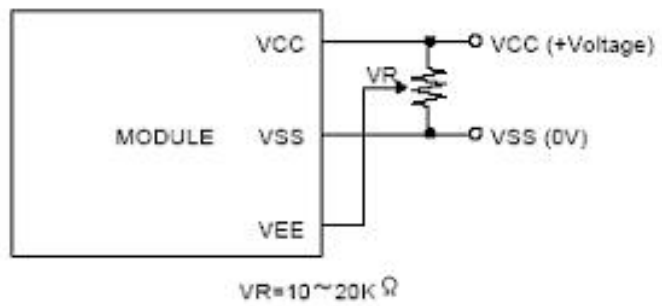


4.3.Block Diagram
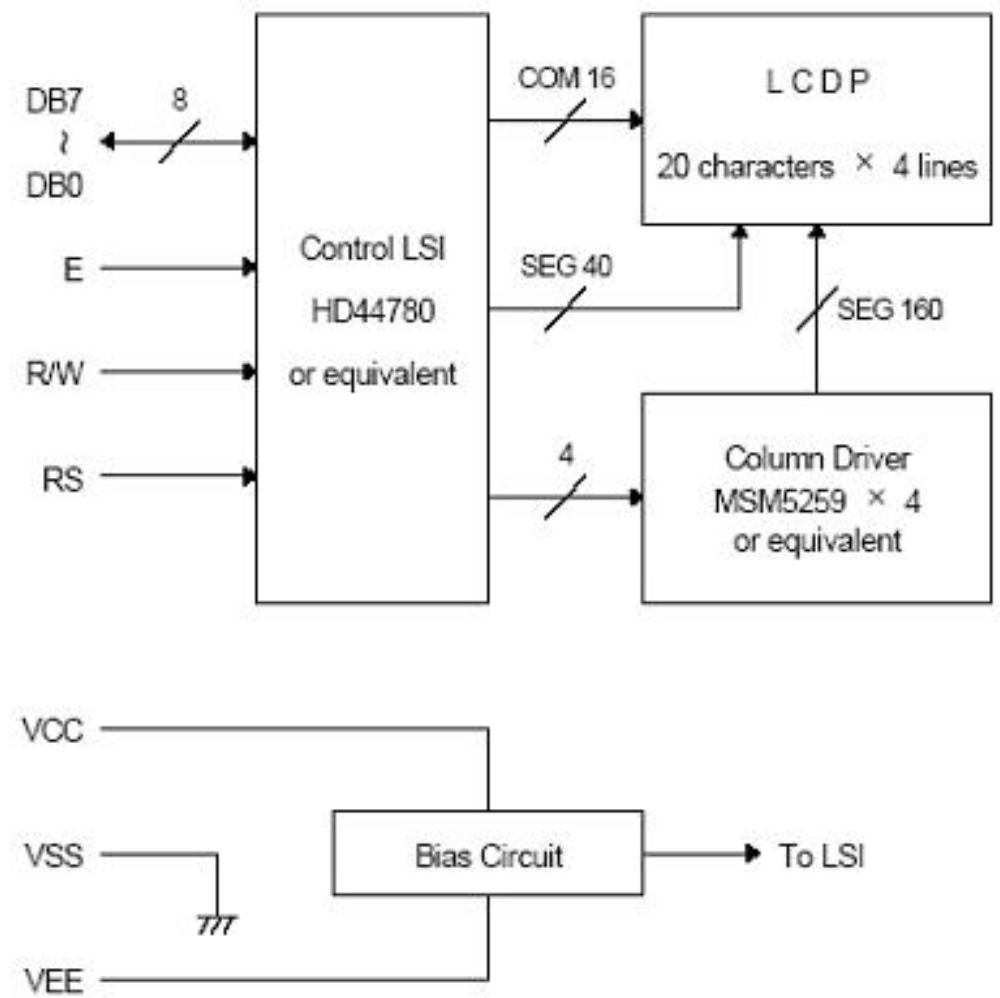
A.A7 PIC12F629 - used for the 1KHz oscillator in Analog Block (IC8)

(From www.microchip.com )

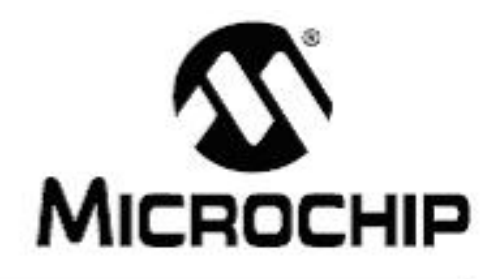

PIC12F629/675

Data Sheet

8-Pin FLASH-Based 8-Bit

CMOS Microcontrollers

Pin Diagrams

8-pin PDIP, SOIC, DFN-S
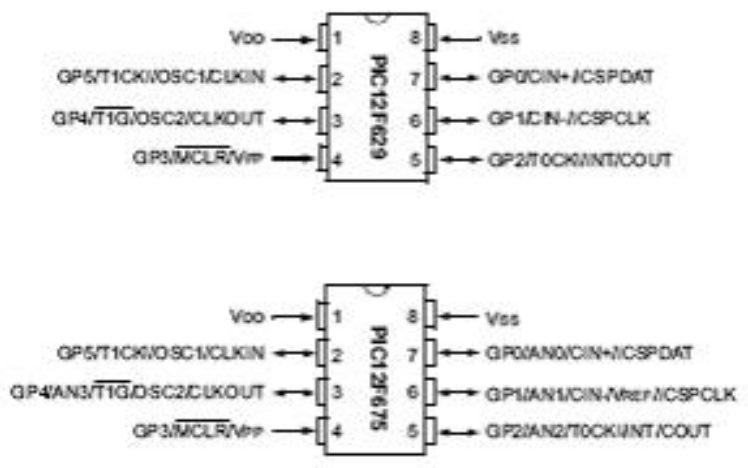
High Performance RISC CPU:

- Only 35 instructions b leam

- All single cycle instructions except branches

- Operating speed:

- DC - $20 \mathrm{MHz}$ osclllabr/dock input

- DC - 200 ns instruction cycle

- Internupt capability

- 8-level deep hardware stack

- Drect, Indirect, and Relative Addressing modes

Special Microcontroller Features:

- Internal and external oscillabr optons

- Predision Internal $4 \mathrm{MHz}$ oscillabe factory calibrated to $\pm 1 \%$

- Extemal Oscillabor support for crystals and resonators

- 5 us wake-up from SLEEP, $3.0 \mathrm{~V}$, typical

- Power saving SLEEP mode

- Wde operating voltage range - $2.0 \mathrm{~V}$ b $5.5 \mathrm{~V}$

- Industial and Extended temperature range

- Low power Power-on Reset (POR)

- Power-up Timer (PWRT) and Osclllabr Start-up Timer (OST)

- Brown-out Detect (BOD)

- Watchdog Timer (WDT) with independent oscillator for rellable operation

- Multiplexed MCLRIInput-pin

- Internupt-on-pin change

- Individual programmable weak pull-ups

- Programmable code protaction

- Hgh Endurance FLASHEEPROM Cell

- 100,000 wite FLASH endurance

- 1,000,000 wrte EEPROM enduranoe

- FLASHData EEPROM Retenton: > 40 years
Low Power Features:

- Standby Cument:

- 1 nA @ 2.0V, typical

- Operating Current:

- $8.5 \mu \mathrm{A} @ 32 \mathrm{kHz}, 2.0 \mathrm{~V}$, typical

- $100 \mu \mathrm{A} @ 1 \mathrm{MHz}, 2.0 \mathrm{~V}$, typical

- Watchdog Timer Current

- 300 nA @ 2.0V, typical

- Timer1 oscillaber current:

- $4 \mu \mathrm{A} @ 32 \mathrm{kHz}, 2.0 \mathrm{~V}$, typical

Peripheral Features:

- 610 pins with individual direction control

- High current sink/ source for direct LED drive

- Analog comparabr module with:

- One analog comparator

- Programmable on-chip comoarator voltage reference (CVefF) module

- Programmable input multplexing from devioe inputs

- Comparator output is extemally accessible

- Analog-to-Dbital Converter module (PIC12F675).

- 10-bit resolution

- Progranmable 4-channel input

- Voltage reference input

- Timeno: 8-bit timericounter with 8-bit programmable prescaler

- Enhanced Timer1:

- 16-bit tmericounter with prescaler

- External Gate Input mode

- Opton to use OSC1 and OSC2 in LP mode as Timert oscillator, if INTOSC mode selected

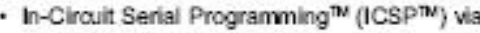
two pins

\begin{tabular}{|c|c|c|c|c|c|c|c|}
\hline \multirow{2}{*}{ Device } & \multirow{2}{*}{$\begin{array}{l}\begin{array}{l}\text { Program } \\
\text { Memory }\end{array} \\
\text { FLASH } \\
\text { (words) }\end{array}$} & \multicolumn{2}{|c|}{ Data Menory } & \multirow{2}{*}{110} & \multirow{2}{*}{$\begin{array}{c}\text { 10-bitA/D } \\
\text { (ch) }\end{array}$} & \multirow{2}{*}{ Comparators } & \multirow{2}{*}{$\begin{array}{l}\text { Timers } \\
\text { \&16-bin }\end{array}$} \\
\hline & & $\begin{array}{l}\text { SRAM } \\
\text { (bytos) }\end{array}$ & $\begin{array}{c}\text { EEPROM } \\
\text { (bytss) }\end{array}$ & & & & \\
\hline PIC12F829 & 1024 & 64 & 128 & 6 & 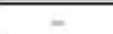 & 1 & $1 / 1$ \\
\hline PIC12F675 & 1024 & 64 & 128 & 6 & 4 & 1 & $1 / 1$ \\
\hline
\end{tabular}




\section{A.A8 PIC18F252 - the" brain" of the Decision block}

(From www.microchip.com )

\section{N11 \\ MICROCHIP \\ 28/40-pin High Performance, Enhanced FLASH Microcontrollers with 10-Bit A/D}

High Performance RISC CPU:

- C compler optinized architecture/nstruction set

- Source code compatible with the PIC16 and PIC17 instruction sets

- Linear program memory addressing to 32 Kbytes - Linear data memory addressing to 1.5 Kbytes

\begin{tabular}{|c|c|c|c|c|}
\hline \multirow{2}{*}{ Device } & \multicolumn{2}{|c|}{$\begin{array}{l}\text { On-Chlp Program } \\
\text { Memory }\end{array}$} & \multirow{2}{*}{$\begin{array}{c}\text { On-Chlp } \\
\text { RAM } \\
\text { (Dytes) }\end{array}$} & \multirow{2}{*}{$\begin{array}{c}\text { Data } \\
\text { EEPROM } \\
\text { (bytes) }\end{array}$} \\
\hline & $\begin{array}{l}\text { FLASH } \\
\text { (byta8) }\end{array}$ & $\begin{array}{c}\text { \# Single Word } \\
\text { Instructions }\end{array}$ & & \\
\hline F/C137242 & $16 \mathrm{~K}$ & 8192 & 768 & 256 \\
\hline PIC18F252 & $32 K$ & 16384 & 1536 & 256 \\
\hline $\mathrm{PIC18F442}$ & $16 \mathrm{~K}$ & 8192 & 768 & 256 \\
\hline PIC18F452 & $32 K$ & 16384 & 1536 & 256 \\
\hline
\end{tabular}

- Up to 10 MIPs operation:

- DC - $40 \mathrm{MHz}$ osc./clock input

- $4 \mathrm{MHz}-10 \mathrm{MHz}$ osciclock input with PLL active

- 16-bit wide instructions, 8-bit wide data path

- Prionty levels for interrupts

- 8 ×8 Single Cycle Hardware Multiplier

Peripheral Features:

- High current sink/source $25 \mathrm{~mA} / 25 \mathrm{~mA}$

- Three external interrupt pins

- Timer0 module: 8-bit/16-bit timericounter with 8-bit programmable prescaler

- Timer1 module: 16-bit timericounter

- Timer2 module: 8-bit timericounter with 8-bit period register (time-base for PWM)

- Timer3 module: 16-bit timericounter

- Secondary oscillator clock option - Timer1/Timer3

- Two Capture/Compare/PWM (CCP) modules.

$C C P$ pins that can be configured as:

- Capture input: capture is 16-bit, max. resolution $6.25 \mathrm{~ns}$ (Tor/16)

- Compare is 16-bit, max. resolution $100 \mathrm{~ns}$ (TCY)

- PWM outout: PWM resolution is 1- to 10-bit, max. PWM freq. Q: 8-bit resolution $=156 \mathrm{kHz}$ 10 -bit resolution $=38 \mathrm{kHz}$

- Master Synchronous Serial Port (MSSP) module. Two modes of operation:

- 3-wire SPI"r (supports all 4 SPI modes)

- $1^{2} \mathrm{C}^{\mathrm{n}}$ Master and Slave mode
Peripheral Features (Continued):

- Addressable USART module:

- Supports RS-485 and RS-232

- Paralel Slave Port (PSP) module

Analog Features:

- Compatible 10-bit Analog-to-Digital Converter module (A/D) wth:

- Fast sampling rate

- Conversion available during SLEEP

- Linearty 1 LSb

- Programmable Low Votage Detection (PLVD)

- Supports interrupt on-Low Voltage Detection

- Programmable Brown-out Reset (BOR)

Special Microcontroller Features:

- 100,000 eraseiwrite cycle Enhanced FLASH program memory typical

- $1,000,000$ eraseiwrite cycle Data EEPROM

memory

- FLASHIData EEPROM Retention: > 40 years

- Sef-reprogrammable under software control

- Power-on Reset (POR), Power-up Timer (PWRT) and Oscillator Start-up Timer (OST)

- Watchdog Timer (WDT) with its own On-Chip RC Oscilator for re lable operation

- Programmable code protection

- Power saving SLEEP mode

- Selectable oscillator options including:

- 4X Phase Lock Loop (of primary oscilator)

- Secondary Oscillator $(32 \mathrm{kHz})$ clock input

- Single supply $5 V$ in-Circuit Serial Programming Th (ICSP TI') via two pins

- In-Circuit Debug (ICD) via two pins

CMOS Technology:

- Low power, high speed FLASHIEEPROM technology

- Fully static design

-Wide operating voltage range $(2.0 \mathrm{~V}$ to $5.5 \mathrm{~V})$

- Industrial and Extended temperature ranges

- Low power consumption:

- <1.6 mA typical @5V, $4 \mathrm{MHz}$

$-25 \mu \mathrm{A}$ typical @ $3 \mathrm{~V}, 32 \mathrm{kHz}$

$-<0.2 \mu$ A typical standby current 


\section{PIC18FXX2}

Pin Diagrams (Cont.'d)

DIP

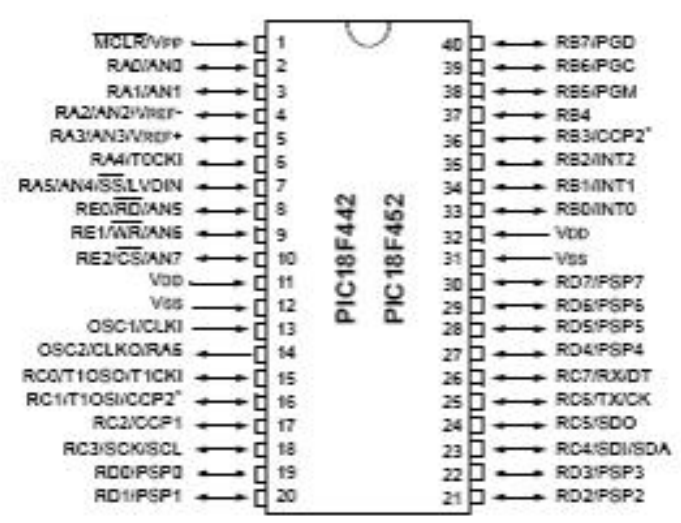

Note: Pin compatble ath 40 -sin Ficiecrx cenices:

DIP, SOIC

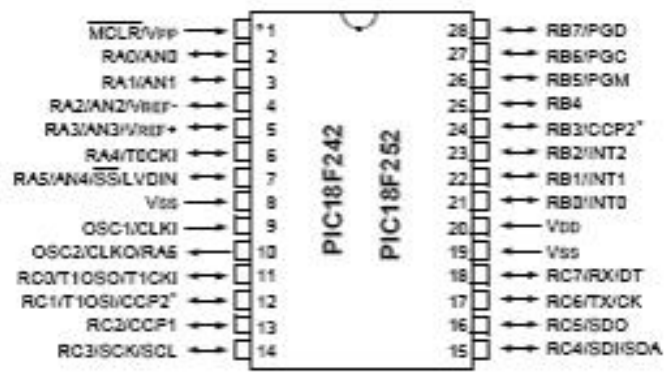

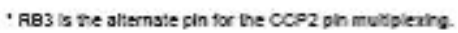




\section{A.A9 Development tools used}

For this project I used MPLAB IDE 6.2 environment and PCW C Compiler IDE, to write, build the projects and compile. To program I used the EPIC programmer and software. EPIC tools are not as advanced as MPLAB ICD2, for instance, because they are not capable of OCP (on-circuit-programming), neither of in-circuit-debugging, but they are very fast and flexible, with fewer settings to make.

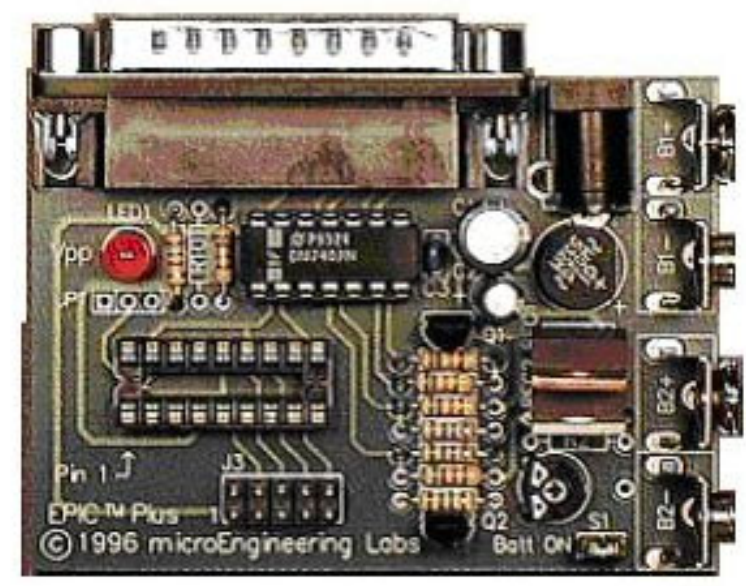

Fig. A.A9.A This is the EPIC programmer from Micro Engineering Labs Inc. It costs $\$ 59.95$.

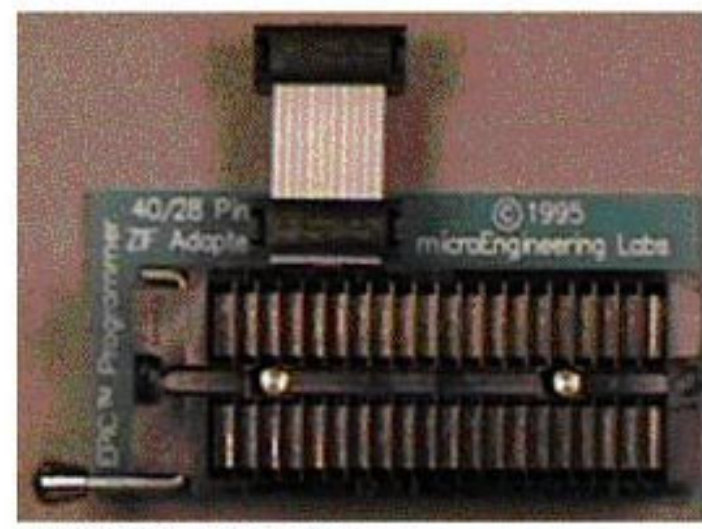

\section{0/28 Pin ZIF Adapter}

Fig.A.A9B The ZIF Adapter helps for programming PIC's with more than 18 pins.

There are a lot of adapters that can be used, according to the PIC MCU used. The 40/28pin adapter satisfied our needs perfectly. The EPIC Programmer needs a parallel-parallel cable that will connect to the LPT1 parallel port of the PC. It also needs a DC adapter, $15 \mathrm{~V} / 500 \mathrm{~mA}$, but can be supplied also by two $9 \mathrm{~V}$ batteries.

The software comes together with the programmer on a floppy disk and is very easy to install. 


\section{APPENDIX B}

\section{A.B1 The Switched Capacitor Filter (SCF)}

(See [24])

This appendix is suppose to be a help in understanding the Chapter5, Paragraph 5.4.

Definition: The SCF is a unique class of active filters, used primarily in:

- voice-band communications

- process telephonic signals

- recognition systems

- speech synthesis.

SCF Advantages

- low power consumption

- occupy very little space (all circuit elements (beside the clock) are in the IC chip)

- used for high Q (quality factor, reflecting the sharpness of the curve) design. Uses cascaded, basic b-quad format active filters, in which the resistors ${ }^{\circledR}$ are replaced with SC (switched capacitors).

SCF disadvantage

- inherently noisy. Their noise comes from aliasing in the sampling process (in reality SCF is an analog sampled data filter) and from the MOS op-amps used, which are more noisy than BJTs (bipolar junction transistors) or BIFET (JFET + BJT).

What to do to minimize the noise in SCFs ?

To minimize the noise in the SCFs satisfy the Nyquist criterion for $\mathrm{A} / \mathrm{D}$ aliasing. The input signals (for the SCF to function properly) they process must contain no significant spectral power above one half of the capacitor switching frequency.

SCF basic functionality

As previously stated, SCF is an active filter, using op-amps in which each resistor is replaced by a MOS capacitor, which is rapidly and synchronously switched by MOS analog switches. Figure AB1 shows how a pair of MOS transistors as analog switches, are driven by a two-phase, non-overlapping clock wave-form.

Note: A heuristic analysis of SCFs treats the "switched capacitor" as a resistor.

In Fig.AB1: - Cs is the MOS capacitor;

- Q1, Q2 are MOSFET transistors as analog switches. 

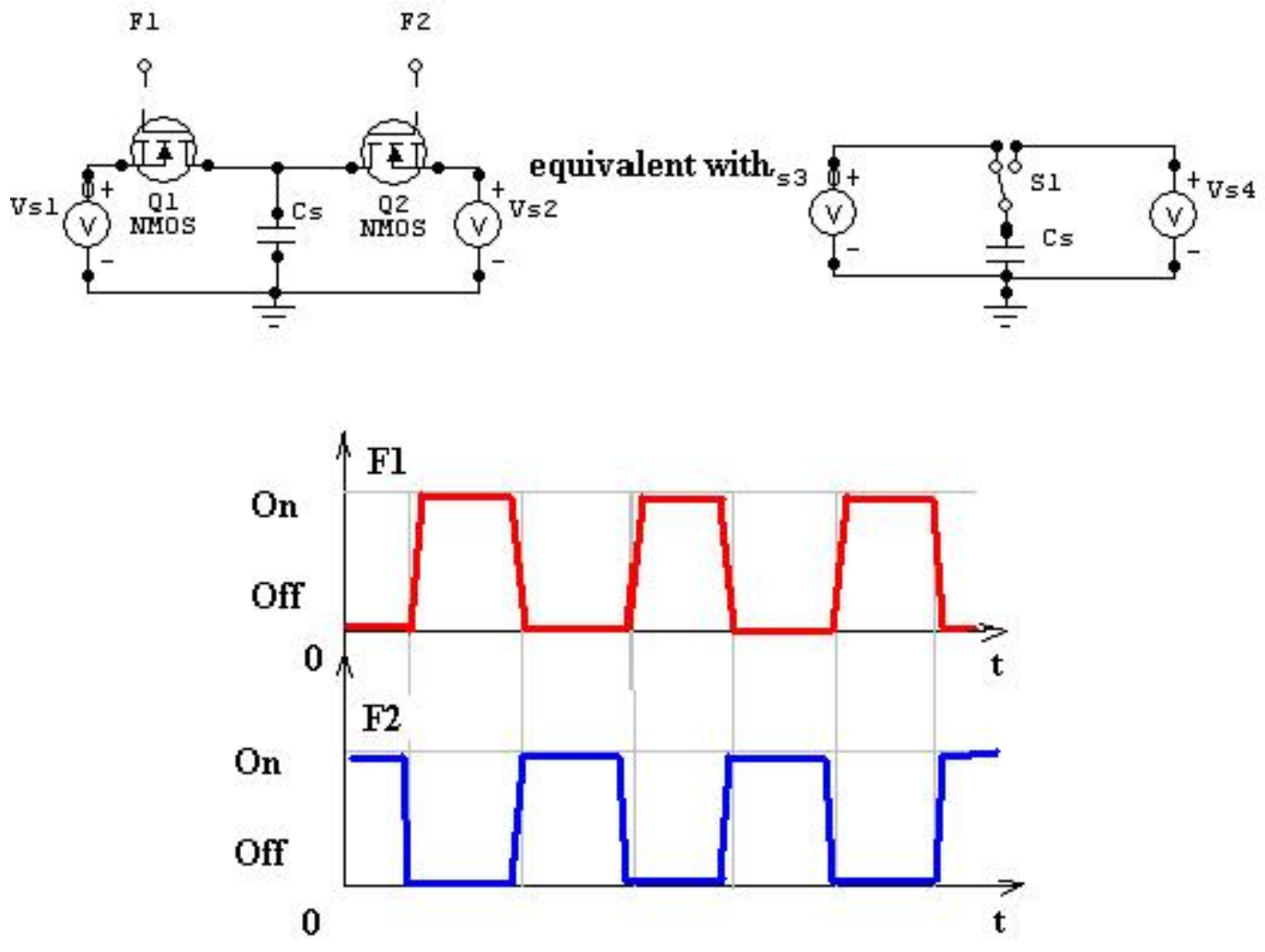

Fig. A.B1: Treating a switched capacitor as a resistor. With red and blue are represented two-phase clock waveforms to switch the MOS transistor

(From [24])

Explanation for fig. AB1: When F1=1 then Cs charges rapidly to Vs1, through Q1. After this Q1 blocks $(\mathrm{F} 1=0)$.

When $\mathrm{F} 2=1$ then $\mathrm{Q} 2$ connects Cs to Vs2 and complete 1 cycle. The net charge transferred through Q2 is: $\Delta \mathrm{q}=\mathrm{Cs}(\mathrm{Vs} 1-\mathrm{Vs} 2)$;

The current transferred in one clock cycle Tc, is:

$$
\begin{aligned}
\mathrm{Ic}=\Delta \mathrm{q} / \Delta \mathrm{t}=[\mathrm{Cs}(\mathrm{Vs} 1-\mathrm{Vs} 2)] / \mathrm{Tc} & =(\mathrm{Vs} 1-\mathrm{Vs} 2) /(1 / \mathrm{fc} * \mathrm{Cs})= \\
& =\mathrm{fc} * \mathrm{Cs}(\mathrm{Vs} 1-\mathrm{Vs} 2) ;
\end{aligned}
$$

but Ic $=(\mathrm{Vs} 1-\mathrm{Vs} 2) / \mathrm{Req}$; then Req $=1 / \mathrm{fc} * \mathrm{Cs}$; meaning that the equivalent resistance depends directly on $\mathrm{Cs}$ (the capacitance of the MOS capacitor) and $\mathrm{fc}=1 / \mathrm{Tc}$, the period of the cycle.

Example : Req values for various SC values (Cs) and various clock frequencies.

\begin{tabular}{|l|l|l|l|}
\hline $\mathrm{Cs}[\mathrm{pF}]$ & $\mathrm{fc} 1=10 \mathrm{e} 4 \mathrm{~Hz}$ & $\mathrm{fc} 2=10 \mathrm{e} 5 \mathrm{~Hz}$ & $\mathrm{fc} 3=10 \mathrm{e} 6 \mathrm{~Hz}$ \\
\hline 0.1 & $10 \mathrm{e} 9[\mathrm{Ohms}]$ & $10 \mathrm{e} 8$ & $10 \mathrm{e} 7$ \\
\hline 1 & $10 \mathrm{e} 8$ & $10 \mathrm{e} 7$ & $10 \mathrm{e} 6$ \\
\hline 10 & $10 \mathrm{e} 7$ & $10 \mathrm{e} 6$ & $10 \mathrm{e} 5$ \\
\hline 100 & $10 \mathrm{e} 6$ & $10 \mathrm{e} 5$ & $10 \mathrm{e} 4$ \\
\hline
\end{tabular}

Observation: While increasing the frequency decrease the Req (equivalent resistance) 


\section{A.B2 Lambert-Beer Law}

(From http://www.photometer.com/en/abc/abc 061.htm )

The scientific basis of absorbance measurement relies on the Lambert-Beer Law.

Named after the two scientists J.H. Lambert (1728-1777) and August Beer (1825-1863), this law states the correlation between the absorbance A, the path length traversed, and the concentration of the absorbent substance:

$\mathrm{A}=\mathrm{k} * \mathrm{c} * \mathrm{~d}$

Where: $\mathbf{c}$ - is the concentration, stated in [mol/1]

$\mathbf{d}-$ is the path length in $[\mathrm{cm}]$

$\mathbf{k}$ - is the relative spectral absorption coefficient ( a substance-specific function of the wavelength).

If monochromatic light is used in the appropriate concentration range, the Lambert-Beer Law is reliable with great accuracy. Consequently, the concentration of a substance dissolved in liquids or gases, can be determined by measuring the absorbance $\mathbf{A}$.

\section{A.B3 Analog and Logic Block PCB design using Eagle 4.1 software}

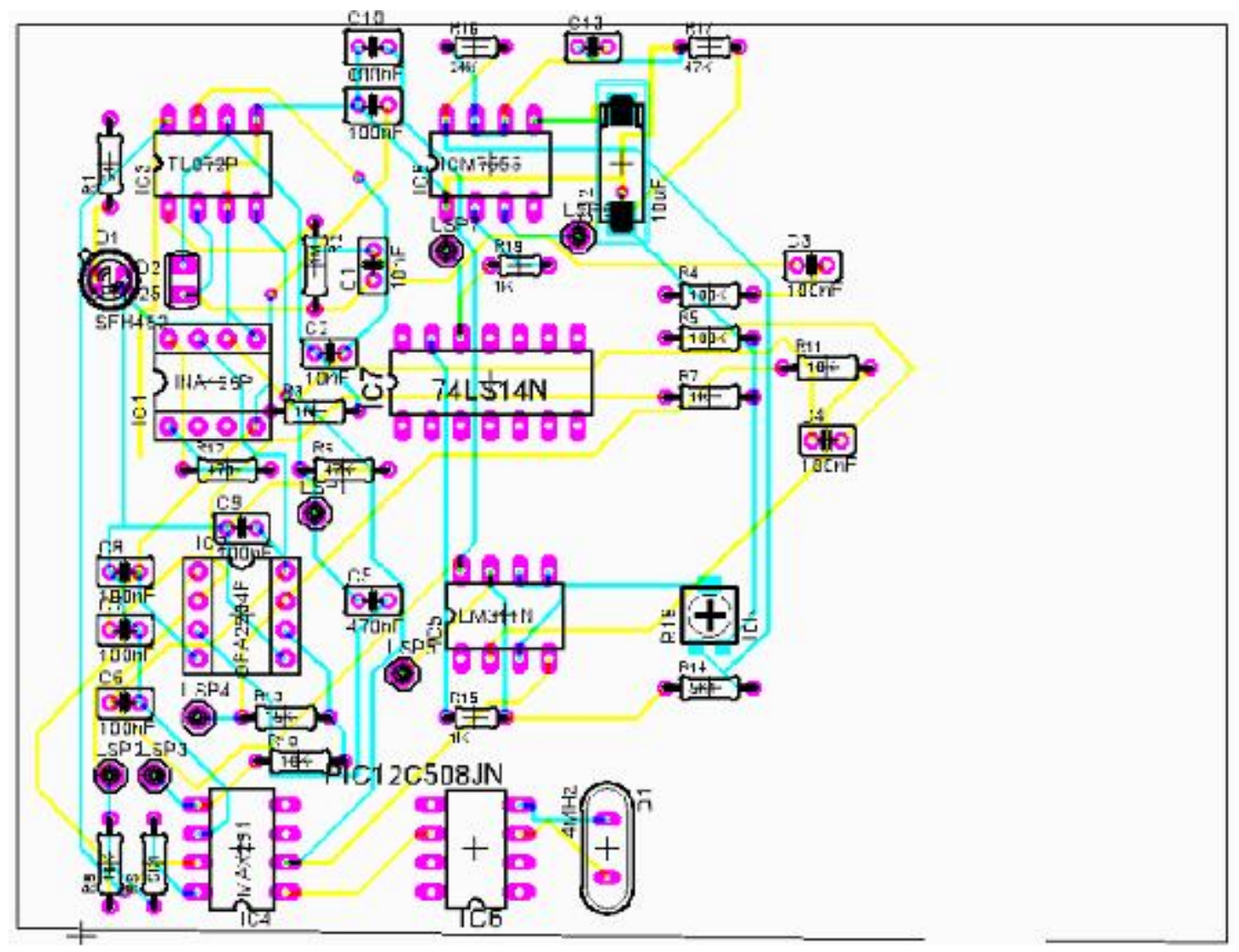

Fig. A.B3 PCB (Printed Circuit Board) built with Eagle 4.11 
The PCB from fig.A.B3 resulted when auto-routing with Eagle 4.11. Theoretically, after the Schematic is done in software, the "erc" program will tell about the errors (there is a sort of a compiler, verifying several rules, and finally giving a report). After getting a no error report, one can switch to the board (from the file menu / Switch to the board) and you can try either routing yourself (that is recommended for this kind of complexity) or try the auto-routing procedure, that can work or not, according to the complexity of the schematic, and the surface of the PCB. Eagle, non-profit version, will work if you can crowd everything on a $60 / 80 \mathrm{~mm}$ PCB, with no MCU involved. After routing and being satisfied with the result, the project has to be saved. The Gerber files were built during the compilation. Once having the Gerber files, a photo-plotter can be used, or more sophisticated tools.

Another solution is to send the Gerber files (and the Drilling Plan) to a company specialized in making PCBs, like PCB123 or Express PCB. The PCBs will be artistically done, usually for an amount of tens of dollars, for a medium complexity. This seems to be too expensive for prototyping, but not for series production.

\section{A.B4 Decision Block PCB design using Protel software}

For Analog and Logic Block PCBs either Circuit Maker or Eagle software were used. For the Decision Block a more sophisticated software was used: Protel DXP. Figures A.B4.A and B are showing the Schematic with the electronic connections of the devices in the block, and the PCB, which is done through the auto-routing method.

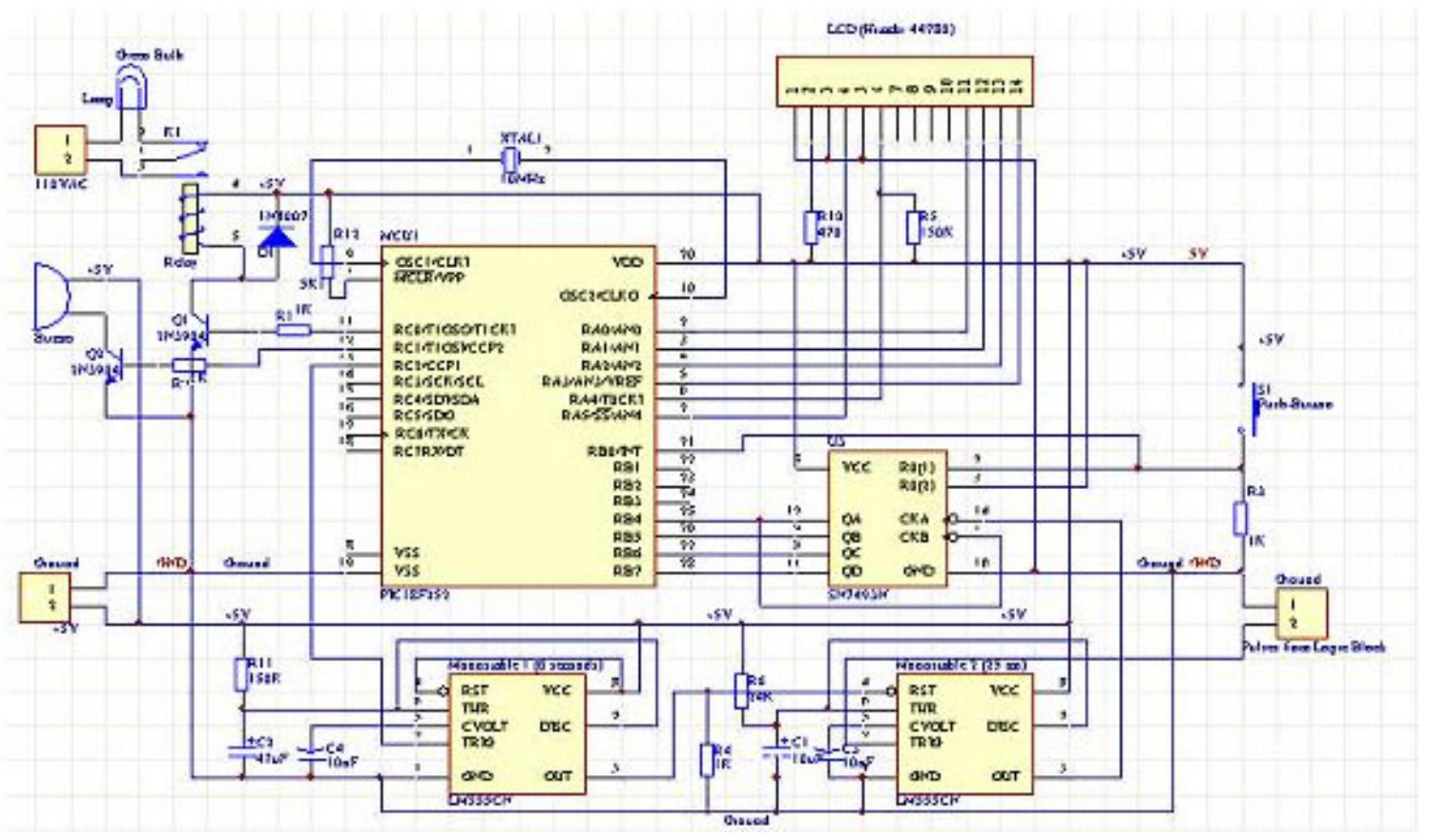

Fig.A.B4.A Schematic circuit done with Protel DXP Software 


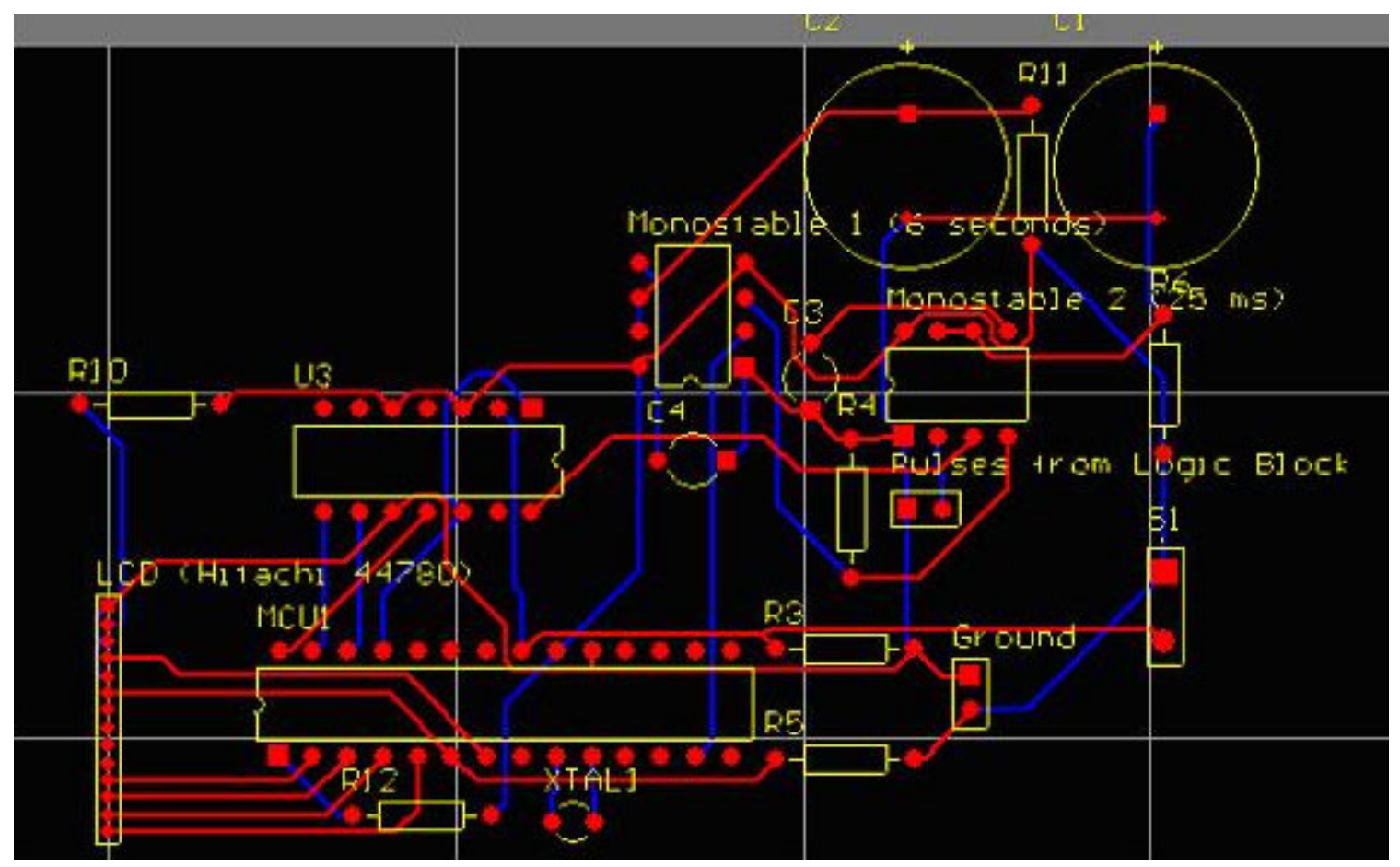

Fig.A.B4.B PCB auto-routed by Protel DXP Software (blue- bottom layer, red- upper layer) 


\section{APPENDIX C}

\section{A.C1. C code for the $1 \mathrm{KHz}$ rectangular oscillator with PIC $12 \mathrm{~F} 629$}

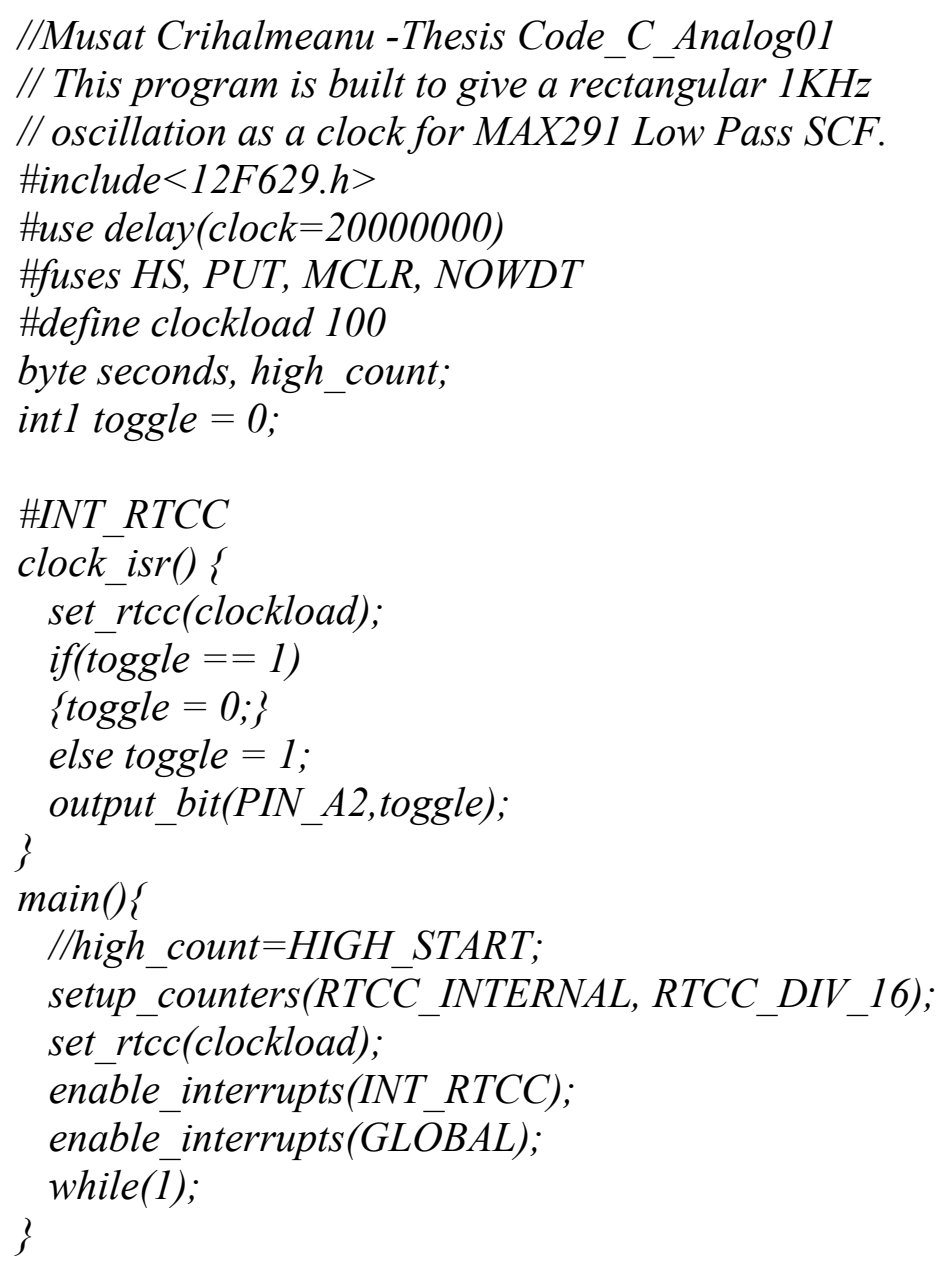

\section{A.C2 C code (short) for the Decision Block, version 1, Roll Over}

//Musat Crihalmeanu Thesis C_Code_Decision_01

//This program contains the $C \bar{c}$ code for the first version, //the Roll Over algorithm, of the Decision Block.

//The CCP1 (capture, compare and PWM) module is used.

//The LCD will display the pulse rate in bpm and the liveness

//resolution

\#include $<18$ F452.h>

\#use delay (clock=4000000)

\#include $<l c d . h>$ 


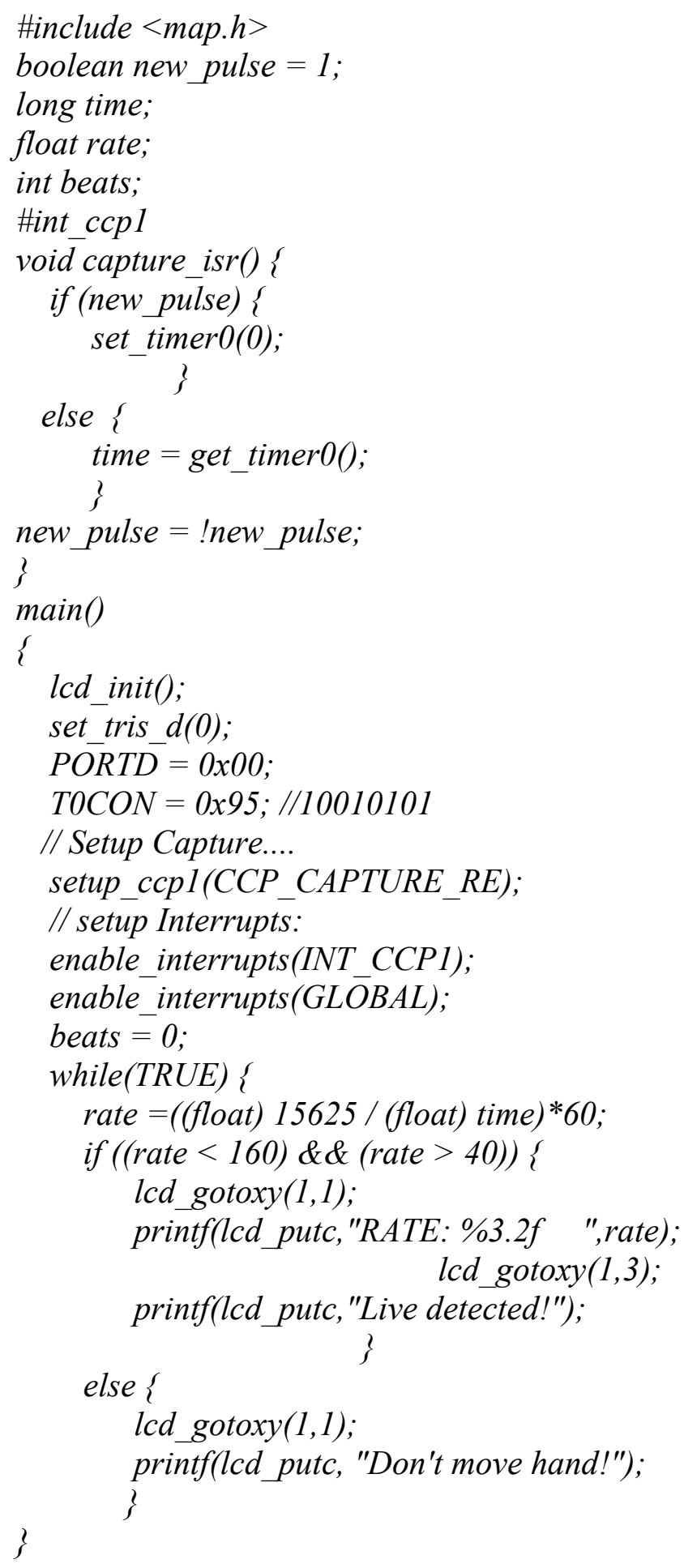




\section{A.C3 C-code for the Decision Block, version 2 (6 seconds sequence)}

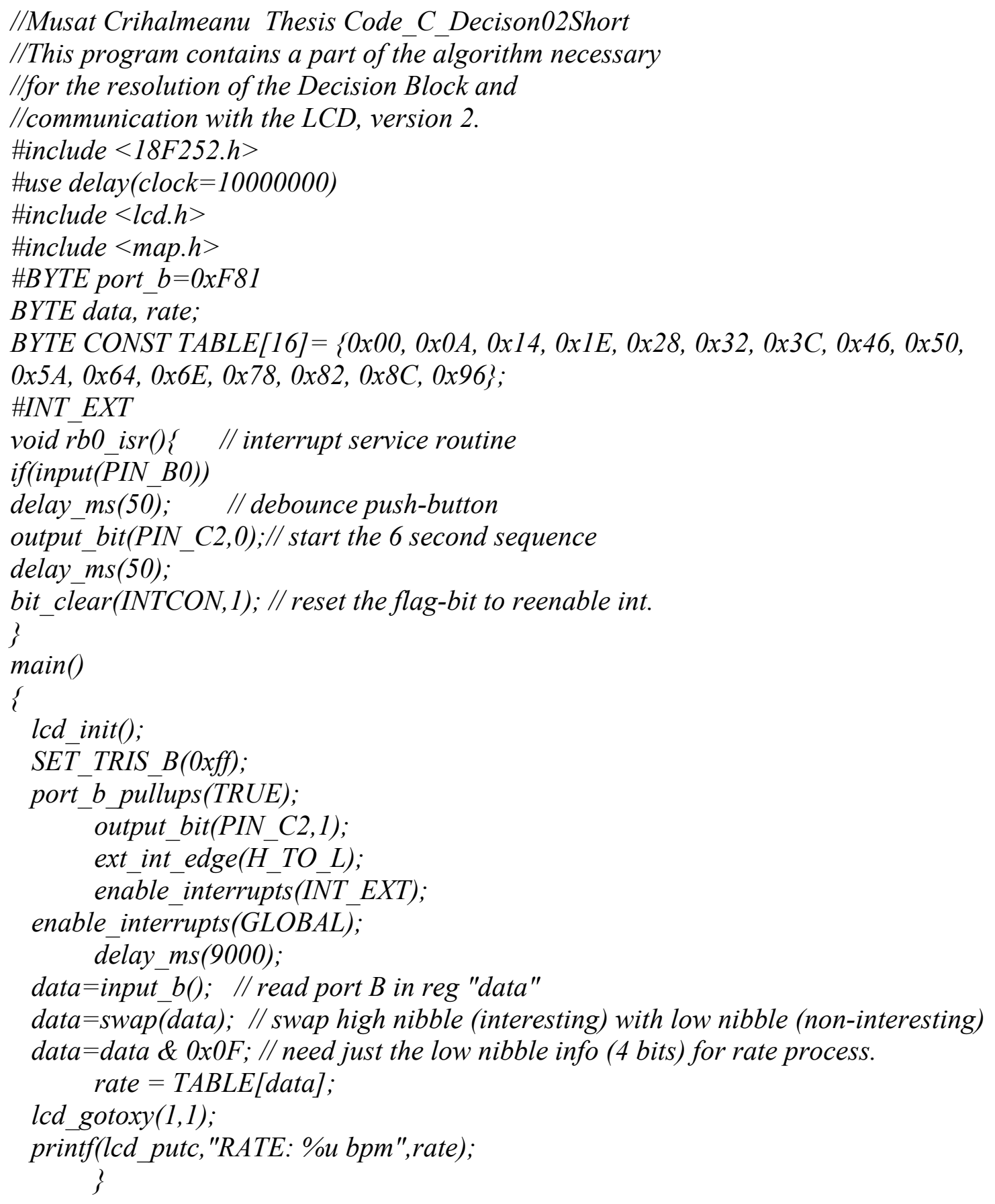




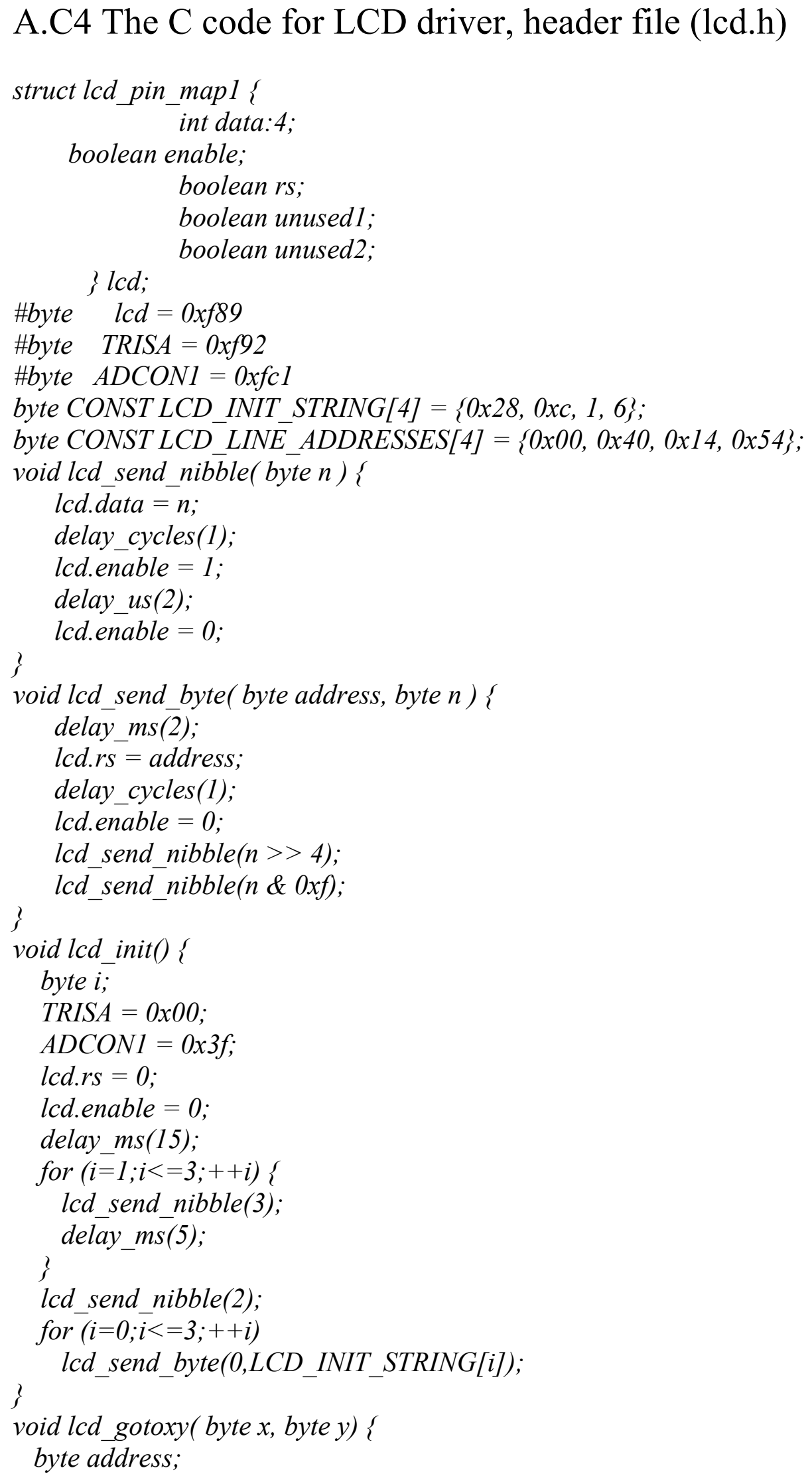




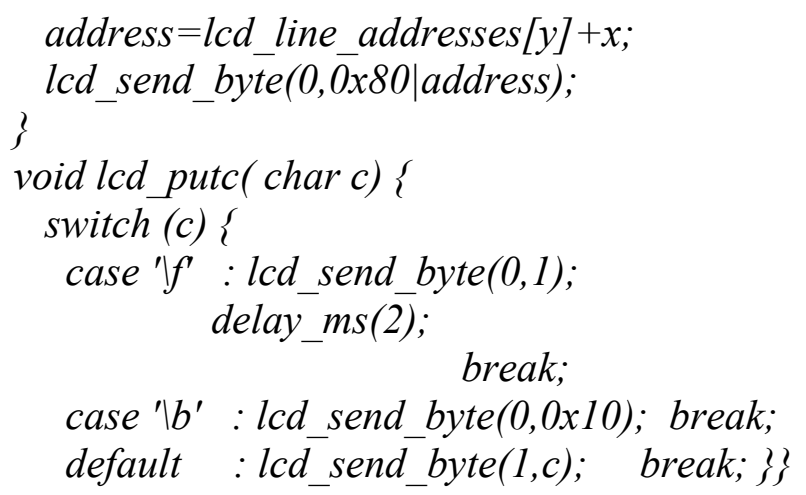

